\title{
NLO evolution of color dipoles in $\mathcal{N}=4 \mathrm{SYM}$
}

\author{
Ian Balitsky ${ }^{\mathrm{a}, \mathrm{b}, *}$, Giovanni A. Chirilli ${ }^{\mathrm{a}, \mathrm{b}}$ \\ a Physics Dept., ODU, Norfolk, VA 23529, United States \\ b Theory Group, Jlab, 12000 Jefferson Ave, Newport News, VA 23606, United States
}

Received 18 May 2009; accepted 1 July 2009

Available online 4 July 2009

\begin{abstract}
High-energy behavior of amplitudes in a gauge theory can be reformulated in terms of the evolution of Wilson-line operators. In the leading logarithmic approximation it is given by the conformally invariant BK equation for the evolution of color dipoles. In QCD, the next-to-leading order BK equation has both conformal and non-conformal parts, the latter providing the running of the coupling constant. To separate the conformally invariant effects from the running-coupling effects, we calculate the NLO evolution of the color dipoles in the conformal $\mathcal{N}=4$ SYM theory. We define the "composite dipole operators" with the rapidity cutoff preserving conformal invariance. The resulting Möbius-invariant kernel for these operators agrees with the forward NLO BFKL calculation of [A.V. Kotikov, L.N. Lipatov, Nucl. Phys. B 582 (2000) 19; A.V. Kotikov, L.N. Lipatov, Nucl. Phys. B 661 (2003) 19; A.V. Kotikov, L.N. Lipatov, Nucl. Phys. B 685 (2004) 405, Erratum].

(C) 2009 Elsevier B.V. All rights reserved.
\end{abstract}

PACS: 12.38.Bx; 12.38.Cy

Keywords: High-energy asymptotics; Evolution of Wilson lines; Conformal invariance

\section{Introduction}

The high-energy scattering in a gauge theory can be described in terms of Wilson lines - infinite gauge factors ordered along the straight lines (see e.g. the review [2]). Indeed, the fast particle moves along its straight-line classical trajectory and the only quantum effect is the eikonal phase factor acquired along this propagation path. In QCD, for fast quark or gluon scattering off some

\footnotetext{
* Corresponding author at: Theory Group, Jlab, 12000 Jefferson Ave, Newport News, VA 23606, United States.

E-mail addresses: balitsky@jlab.org (I. Balitsky), chirilli@jlab.org (G.A. Chirilli).
} 
target, this eikonal phase factor is a Wilson line - an infinite gauge link ordered along the straight line collinear to particle's velocity $n^{\mu}$ :

$$
U^{\eta}\left(x_{\perp}\right)=\operatorname{Pexp}\left\{i g \int_{-\infty}^{\infty} d u n_{\mu} A^{\mu}\left(u n+x_{\perp}\right)\right\} .
$$

Here $A_{\mu}$ is the gluon field of the target, $x_{\perp}$ is the transverse position of the particle which remains unchanged throughout the collision, and the index $\eta$ labels the rapidity of the particle.

The high-energy behavior of QCD amplitudes can be studied in the framework of the evolution of color dipoles. Let us consider the small- $x$ behavior of structure functions of deep inelastic scattering (DIS). At high energies the virtual photon decomposes into quark and antiquark which propagate along the straight lines separated by transverse distance and form a color dipole two-Wilson-line operator.

$$
\hat{\mathcal{U}}^{\eta}\left(x_{\perp}, y_{\perp}\right)=1-\frac{1}{N_{c}} \operatorname{tr}\left\{\hat{U}^{\eta}\left(x_{\perp}\right) \hat{U}^{\dagger \eta}\left(y_{\perp}\right)\right\} .
$$

The energy dependence of the structure function is then translated into the dependence of the color dipole on the rapidity $\eta$. There are two ways to restrict the rapidity of Wilson lines: one can consider Wilson lines with the support line collinear to the velocity of the fast-moving particle or one can take the light-like Wilson line and cut the rapidity integrals "by hand". While the former method appears to be more natural, it is technically simpler to get the conformal results with the latter method of "rigid cutoff" in the longitudinal direction.

Thus, the small- $x$ behavior of the structure functions is governed by the rapidity evolution of color dipoles $[3,4]$. At relatively high energies and for sufficiently small dipoles we can use the leading logarithmic approximation (LLA) where $\alpha_{s} \ll 1, \alpha_{s} \ln x_{B} \sim 1$ and get the non-linear BK evolution equation for the color dipoles $[5,6]$ :

$$
\begin{aligned}
\frac{d}{d \eta} \hat{\mathcal{U}}^{\eta}\left(z_{1}, z_{2}\right)= & \frac{\alpha_{s} N_{c}}{2 \pi^{2}} \int d^{2} z_{3} \frac{z_{12}^{2}}{z_{13}^{2} z_{23}^{2}}\left[\hat{\mathcal{U}}^{\eta}\left(z_{1}, z_{3}\right)+\hat{\mathcal{U}}^{\eta}\left(z_{3}, z_{2}\right)\right. \\
& \left.-\hat{\mathcal{U}}^{\eta}\left(z_{1}, z_{3}\right)-\hat{\mathcal{U}}^{\eta}\left(z_{1}, z_{3}\right) \hat{\mathcal{U}}^{\eta}\left(z_{3}, z_{2}\right)\right],
\end{aligned}
$$

where $\eta=\ln \frac{1}{x_{B}}$ and $z_{12} \equiv z_{1}-z_{2}$, etc. (As usual, we denote operators by "hat".) The first three terms correspond to the linear BFKL evolution [7] and describe the parton emission while the last term is responsible for the parton annihilation. For sufficiently low $x_{B}$ the parton emission balances the parton annihilation so the partons reach the state of saturation [8] with the characteristic transverse momentum $Q_{s}$ growing with energy $1 / x_{B}$ (for a review, see [9]).

It is easy to see that the BK equation (3) is conformally invariant in the two-dimensional space. This follows from the conformal invariance of the light-like Wilson lines. Indeed, the Wilson line

$$
U\left(x_{\perp}\right)=\operatorname{Pexp}\left\{i g \int_{-\infty}^{\infty} d x^{+} A_{+}\left(x^{+}, x_{\perp}\right)\right\}
$$

is invariant under the inversion $x^{\mu} \rightarrow x^{\mu} / x^{2}$ (with respect to the point with zero (-) component). Indeed, $\left(x^{+}, x_{\perp}\right)^{2}=-x_{\perp}^{2}$ so after the inversion $x_{\perp} \rightarrow x_{\perp} / x_{\perp}^{2}$ and $x^{+} \rightarrow x^{+} / x_{\perp}^{2}$ and therefore

$$
U\left(x_{\perp}\right) \rightarrow \operatorname{Pexp}\left\{i g \int_{-\infty}^{\infty} d \frac{x^{+}}{x_{\perp}^{2}} A_{+}\left(\frac{x^{+}}{x_{\perp}^{2}}, x_{\perp}\right)\right\}=U\left(x_{\perp} / x_{\perp}^{2}\right) .
$$


It is easy to check that the Wilson line operators lie in the standard representation of the conformal Möbius group $\operatorname{SL}(2, C)$ with conformal spin 0 (see Appendix A). It should be mentioned that the conformal invariance of the linear BFKL equation was first proved in Ref. [10].

The NLO evolution of color dipole in QCD [11] is not expected to be Möbius-invariant due to the conformal anomaly leading to dimensional transmutation and running coupling constant. However, the NLO BK equation in QCD [11] has an additional term violating Möbius invariance and not related to the conformal anomaly. To understand the relation between the high-energy behavior of amplitudes and Möbius invariance of Wilson lines, it is instructive to consider the conformally invariant $\mathcal{N}=4$ super-Yang-Mils theory. This theory was intensively studied in recent years due to the fact that at large coupling constants it is dual to the IIB string theory in the $\mathrm{AdS}_{5}$ background. In the light-cone limit, the contribution of scalar operators to MaldacenaWilson line [12] vanishes so one has the usual Wilson line constructed from gauge fields and therefore the LLA evolution equation for color dipoles in the $\mathcal{N}=4 \mathrm{SYM}$ has the same form as (3). At the NLO level, the contributions from gluino and scalar loops enter the picture.

As we mentioned above, formally the light-like Wilson lines are Möbius-invariant. Unfortunately, the light-like Wilson lines are divergent in the longitudinal direction and moreover, it is exactly the evolution equation with respect to this longitudinal cutoff which governs the highenergy behavior of amplitudes. At present, it is not known how to find the conformally invariant cutoff in the longitudinal direction. When we use the non-invariant cutoff we expect, as usual, the invariance to hold in the leading order but to be violated in higher orders in perturbation theory. In our calculation we restrict the longitudinal momentum of the gluons composing Wilson lines, and with this non-invariant cutoff the NLO evolution equation in QCD has extra non-conformal parts not related to the running of coupling constant. Similarly, there will be non-conformal parts coming from the longitudinal cutoff of Wilson lines in the $\mathcal{N}=4 \mathrm{SYM}$ equation. We will demonstrate below that it is possible to construct the "composite conformal dipole operator" (order by order in perturbation theory) which mimics the conformal cutoff in the longitudinal direction so the corresponding evolution equation has no extra non-conformal parts. This is similar to the construction of the composite renormalized local operator in the case when the UV cutoff does not respect the symmetries of the bare operator - in this case the symmetry of the UV-regularized operator is preserved order by order in perturbation theory by subtraction of the symmetry-restoring counterterms.

Let us present our result for the NLO evolution of the color dipole in the adjoint representation (hereafter we use notations $z_{i j} \equiv z_{i}-z_{j}$ and $\left(T^{a}\right)_{b c}=-i f^{a b c}$ )

$$
\begin{aligned}
\frac{d}{d \eta}[ & \left.\operatorname{Tr}\left\{\hat{U}_{z_{1}}^{\eta} \hat{U}_{z_{2}}^{\dagger \eta}\right\}\right]^{\mathrm{conf}} \\
= & \frac{\alpha_{s}}{\pi^{2}} \int d^{2} z_{3} \frac{z_{12}^{2}}{z_{13}^{2} z_{23}^{2}}\left[1-\frac{\alpha_{s} N_{c}}{4 \pi} \frac{\pi^{2}}{3}\right]\left[\operatorname{Tr}\left\{T^{a} \hat{U}_{z_{1}}^{\eta} \hat{U}_{z_{3}}^{\dagger \eta} T^{a} \hat{U}_{z_{3}} \hat{U}_{z_{2}}^{\dagger \eta}\right\}-N_{c} \operatorname{Tr}\left\{\hat{U}_{z_{1}}^{\eta} \hat{U}_{z_{2}}^{\dagger \eta}\right\}\right]^{\mathrm{conf}} \\
& -\frac{\alpha_{s}^{2}}{4 \pi^{4}} \int d^{2} z_{3} d^{2} z_{4} \frac{z_{12}^{2}}{z_{13}^{2} z_{24}^{2} z_{34}^{2}}\left\{2 \ln \frac{z_{12}^{2} z_{34}^{2}}{z_{14}^{2} z_{23}^{2}}+\left[1+\frac{z_{12}^{2} z_{34}^{2}}{z_{13}^{2} z_{24}^{2}-z_{14}^{2} z_{23}^{2}}\right] \ln \frac{z_{13}^{2} z_{24}^{2}}{z_{14}^{2} z_{23}^{2}}\right\} \\
& \times \operatorname{Tr}\left\{\left[T^{a}, T^{b}\right] \hat{U}_{z_{1}}^{\eta} T^{a^{\prime}} T^{b^{\prime}} \hat{U}_{z_{2}}^{\dagger \eta}+T^{b} T^{a} \hat{U}_{z_{1}}^{\eta}\left[T^{b^{\prime}}, T^{a^{\prime}}\right] \hat{U}_{z_{2}}^{\dagger \eta}\right\}\left[\left(\hat{U}_{z_{3}}^{\eta}\right)^{a a^{\prime}}\left(\hat{U}_{z_{4}}^{\eta}\right)^{b b^{\prime}}\right. \\
& \left.-\left(z_{4} \rightarrow z_{3}\right)\right],
\end{aligned}
$$

where 


$$
\begin{aligned}
{\left[\operatorname{Tr}\left\{\hat{U}_{z_{1}}^{\eta} \hat{U}_{z_{2}}^{\dagger \eta}\right\}\right]^{\mathrm{conf}}=} & \operatorname{Tr}\left\{\hat{U}_{z_{1}}^{\eta} \hat{U}_{z_{2}}^{\dagger \eta}\right\}+\frac{\alpha_{s}}{2 \pi^{2}} \int d^{2} z_{3} \frac{z_{12}^{2}}{z_{13}^{2} z_{23}^{2}}\left[\operatorname{Tr}\left\{T^{n} \hat{U}_{z_{1}}^{\eta} \hat{U}_{z_{3}}^{\dagger \eta} T^{n} \hat{U}_{z_{3}}^{\eta} \hat{U}_{z_{2}}^{\dagger \eta}\right\}\right. \\
& \left.-N_{c} \operatorname{Tr}\left\{\hat{U}_{z_{1}}^{\eta} \hat{U}_{z_{2}}^{\dagger \eta}\right\}\right] \ln \frac{a z_{12}^{2}}{z_{13}^{2} z_{23}^{2}}
\end{aligned}
$$

is the "composite dipole" with the conformal longitudinal cutoff in the next-to-leading order and $a$ is an arbitrary dimensional constant. (Similar expression for the conformal two-dipole operator in the r.h.s. of this equation is presented below, see Eq. (48).) In fact, $a(\eta)=a e^{\eta}$ plays the same role for the rapidity evolution as $\mu^{2}$ for the usual DGLAP evolution: the derivative $\frac{d}{d a}$ gives the evolution equation (6). The kernel in the r.h.s. of Eq. (6) is obviously Möbius-invariant since it depends on two four-point conformal ratios $\frac{z_{13}^{2} z_{24}^{2}}{z_{14}^{2} z_{23}^{2}}$ and $\frac{z_{12}^{2} z_{34}^{2}}{z_{13}^{2} z_{24}^{2}}$. We will also demonstrate that Eq. (6) agrees with forward NLO BFKL calculation of Ref. [1].

The paper is organized as follows. In Section 2 we remind the derivation of the BK equation in the leading order in $\alpha_{s}$. In Section 3, which is central to the paper, we calculate the scalar and gluino contributions to the small- $x$ evolution of color dipoles and assemble the NLO BK kernel in $\mathcal{N}=4$ SYM. We rewrite the NLO BK kernel in the conformal form (6) in Section 4 and compare our results with the NLO BFKL calculations in $\mathcal{N}=4$ SYM in Section 5. In Section 6 we derive the NLO BK equation for the composite dipoles in the fundamental representation, both in $\mathcal{N}=4$ SYM and in QCD. The Möbius group for the light-like Wilson lines is presented in Appendix A while in Appendix B we find the explicit form (7) of the composite conformal dipole operator by calculating the appropriate impact factor. In Appendix $\mathrm{C}$, we find the leading-order evolution for the four-Wilson-line operator, and Appendices D and E contain some technical calculations which may distract readers from main discussion.

\section{Derivation of the $B K$ equation}

Before discussing the small- $x$ evolution of color dipole in the next-to-leading approximation it is instructive to recall the derivation of the leading-order (BK) evolution equation.

For the NLO calculation we use the lightcone gauge $p_{2}^{\mu} A_{\mu}=0$. In addition we find it convenient to use the "rigid cutoff" prescription in the longitudinal direction. We consider the light-like dipoles (in the $p_{1}$ direction) and impose the cutoff on the maximal $\alpha$ emitted by any gluon from the Wilson lines so

$$
\begin{aligned}
& U_{x}^{\eta}=\operatorname{Pexp}\left[i g \int_{-\infty}^{\infty} d u p_{1}^{\mu} A_{\mu}^{\eta}\left(u p_{1}+x_{\perp}\right)\right], \\
& A_{\mu}^{\eta}(x)=\int d^{4} k \theta\left(e^{\eta}-\left|\alpha_{k}\right|\right) e^{-i k \cdot x} A_{\mu}(k)
\end{aligned}
$$

(hereafter we use the $\hbar$-inspired notation $d^{n} p \equiv \frac{d^{n} p}{(2 \pi)^{n}}$ for brevity). Note that the cutoff (8) respects the unitarity of Wilson lines $\left(U^{\eta} U^{\dagger \eta}=1\right)$.

The momenta $p_{1}$ and $p_{2}$ are the light-like vectors such that $q=p_{1}-x_{B} p_{2}$ and $p=p_{2}+\frac{m^{2}}{s} p_{1}$ where $p$ is the momentum of the target and $m$ is the mass. Throughout the paper, we use the Sudakov variables $p=\alpha p_{1}+\beta p_{2}+p_{\perp}$ and the notations $x_{\bullet} \equiv x_{\mu} p_{1}^{\mu}$ and $x_{*} \equiv x_{\mu} p_{2}^{\mu}$ related to the light-cone coordinates: $x_{*}=x^{+} \sqrt{s / 2}, x_{\bullet}=x^{-} \sqrt{s / 2}$. Our metric is $(1,-1,-1,-1)$ so $p^{2}=\alpha \beta s-p_{\perp}^{2}$ and $x^{2}=\frac{4}{s} x_{\bullet} x_{*}-x_{\perp}^{2}$. 


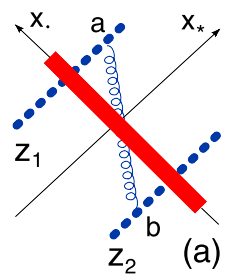

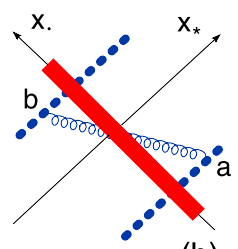

(b)

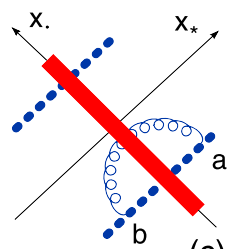

(c)

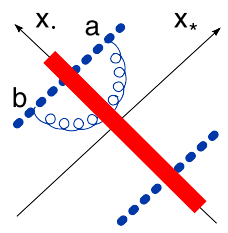

(d)

Fig. 1. Leading-order diagrams for the small- $x$ evolution of color dipole. Gauge links are denoted by dotted lines.

To find the evolution of the color dipole (2) with respect to the slope of the Wilson lines in the leading log approximation we consider the matrix element of the color dipole between (arbitrary) target states and integrate over the gluons with rapidities $\eta_{1}>\eta>\eta_{2}=\eta_{1}-\Delta \eta$ leaving the gluons with $\eta<\eta_{2}$ as a background field (to be integrated over later). In the frame of gluons with $\eta \sim \eta_{1}$ the fields with $\eta<\eta_{2}$ shrink to a pancake and we obtain the four diagrams shown in Fig. 1. Technically, to find the kernel in the leading-order approximation we write down the general form of the operator equation for the evolution of the color dipole

$$
\frac{d}{d \eta} \operatorname{Tr}\left\{\hat{U}_{z_{1}}^{\eta} \hat{U}_{z_{2}}^{\dagger \dagger}\right\}=K_{\mathrm{LO}} \operatorname{Tr}\left\{\hat{U}_{z_{1}}^{\eta} \hat{U}_{z_{2}}^{\dagger \eta}\right\}+\cdots
$$

(where dots stand for the higher orders of the expansion) and calculate the 1.h.s. of Eq. (9) in the shock-wave background

$$
\frac{d}{d \eta}\left\langle\operatorname{Tr}\left\{\hat{U}_{z_{1}}^{\eta} \hat{U}_{z_{2}}^{\dagger \eta}\right\}\right\rangle_{\text {shockwave }}=\left\langle K_{\mathrm{LO}} \operatorname{Tr}\left\{\hat{U}_{z_{1}}^{\eta} \hat{U}_{z_{2}}^{\dagger \eta}\right\}\right\rangle_{\text {shockwave }} .
$$

In what follows we replace $\langle\cdots\rangle_{\text {shockwave }}$ by $\langle\cdots\rangle$ for brevity.

With future NLO computation in view, we will perform the leading-order calculation in the lightcone gauge $p_{2}^{\mu} A_{\mu}=0$. The gluon propagator in a shock-wave external field has the form $[13,14]$

$$
\begin{aligned}
& \left\langle\hat{A}_{\mu}^{a}(x) \hat{A}_{v}^{b}(y)\right\rangle \\
& =\theta\left(x_{*} y_{*}\right) \delta^{a b} \frac{s}{2} \int d \alpha d \beta\left(x_{\perp}\left|\frac{d_{\mu \nu}}{i\left(\alpha \beta s-p_{\perp}^{2}+i \epsilon\right)}\right| y_{\perp}\right) \\
& -\theta\left(x_{*}\right) \theta\left(-y_{*}\right) \int_{0}^{\infty} d \alpha \frac{e^{-i \alpha(x-y)}}{2 \alpha}\left(x_{\perp} \mid e^{-i \frac{p_{\perp}^{2}}{\alpha s} x_{*}}\left[g_{\mu \xi}^{\perp}-\frac{2}{\alpha s}\left(p_{\mu}^{\perp} p_{2 \xi}+p_{2 \mu} p_{\xi}^{\perp}\right)\right]\right. \\
& \left.\times U^{a b}\left[g_{v}^{\perp \xi}-\frac{2}{\alpha s}\left(p_{2}^{\xi} p_{v}^{\perp}+p_{2 v} p_{\perp}^{\xi}\right)\right] e^{i \frac{p_{\perp}^{2}}{\alpha s} y_{*}} \mid y_{\perp}\right) \\
& -\theta\left(-x_{*}\right) \theta\left(y_{*}\right) \int_{0}^{\infty} \pi \alpha \frac{e^{i \alpha(x-y)}}{2 \alpha}\left(x_{\perp} \mid e^{i \frac{p_{\perp}^{2}}{\alpha s}} x_{*}\left[g_{\mu \xi}^{\perp}-\frac{2}{\alpha s}\left(p_{\mu}^{\perp} p_{2 \xi}+p_{2 \mu} p_{\xi}^{\perp}\right)\right]\right. \\
& \left.\times U^{\dagger a b}\left[g_{v}^{\perp \xi}-\frac{2}{\alpha s}\left(p_{2}^{\xi} p_{v}^{\perp}+p_{2 v} p_{\perp}^{\xi}\right)\right] e^{-i \frac{p_{\perp}^{2}}{\alpha s} y_{*}} \mid y_{\perp}\right),
\end{aligned}
$$

where

$$
d_{\mu v}(k) \equiv g_{\mu \nu}^{\perp}-\frac{2}{s \alpha}\left(k_{\mu}^{\perp} p_{2 v}+k_{v}^{\perp} p_{2 \mu}\right)-\frac{4 \beta}{s \alpha} p_{2 \mu} p_{2 v} .
$$


Hereafter we use Schwinger's notations $\left(x_{\perp}\left|F\left(p_{\perp}\right)\right| y_{\perp}\right) \equiv \int d p e^{i(p, x-y) \perp} F\left(p_{\perp}\right)$ (the scalar product of the four-dimensional vectors in our notations is $\left.x \cdot y=\frac{2}{s}\left(x_{*} y_{\bullet}+x_{*} y_{\bullet}\right)-(x, y)_{\perp}\right)$. Note that the interaction with the shock wave does not change the $\alpha$-component of the gluon momentum.

We obtain

$$
\begin{aligned}
& g^{2} \int_{0}^{\infty} d u \int_{-\infty}^{0} d v\left\langle\hat{A}_{\bullet}^{a, \eta_{1}}\left(u p_{1}+x_{\perp}\right) \hat{A}_{\bullet}^{b, \eta_{1}}\left(v p_{1}+y_{\perp}\right)\right\rangle_{\mathrm{Fig} .1 \mathrm{a}} \\
& \quad=-4 \alpha_{S} \int_{0}^{e^{\eta_{1}}} \frac{d \alpha}{\alpha}\left(x_{\perp}\left|\frac{p_{i}}{p_{\perp}^{2}-i \epsilon} U^{a b} \frac{p_{i}}{p_{\perp}^{2}-i \epsilon}\right| y_{\perp}\right) .
\end{aligned}
$$

Formally, the integral over $\alpha$ diverges at the lower limit, but since we integrate over the rapidities $\eta>\eta_{2}$ we get (in the LLA)

$$
\begin{aligned}
& g^{2} \int_{0}^{\infty} d u \int_{-\infty}^{0} d v\left\langle\hat{A}_{\bullet}^{a, \eta_{1}}\left(u p_{1}+x_{\perp}\right) \hat{A}_{\bullet}^{b, \eta_{1}}\left(v p_{1}+y_{\perp}\right)\right\rangle_{\text {Fig. 1a }} \\
& \quad=-4 \alpha_{s} \Delta \eta\left(x_{\perp}\left|\frac{p_{i}}{p_{\perp}^{2}} U^{a b} \frac{p_{i}}{p_{\perp}^{2}}\right| y_{\perp}\right)
\end{aligned}
$$

and therefore

$$
\left\langle\hat{U}_{z_{1}}^{\eta} \otimes \hat{U}_{z_{2}}^{\dagger \eta}\right\rangle_{\text {Fig. 1a }}^{\eta_{1}}=-\frac{\alpha_{s}}{\pi^{2}} \Delta \eta\left(t^{a} U_{z_{1}} \otimes t^{b} U_{z_{2}}^{\dagger}\right) \int d^{2} z_{3} \frac{\left(z_{13}, z_{23}\right)}{z_{13}^{2} z_{23}^{2}} U_{z_{3}}^{a b} .
$$

The contribution of the diagram in Fig. 1b is obtained from Eq. (15) by the replacement $t^{a} U_{z_{1}} \otimes$ $t^{b} U_{z_{2}}^{\dagger} \rightarrow U_{z_{1}} t^{b} \otimes U_{z_{2}}^{\dagger} t^{a}, z_{2} \leftrightarrow z_{1}$ and the two remaining diagrams are obtained from Eq. (14) by taking $z_{2}=z_{1}$ (Fig. 1c) and $z_{1}=z_{2}$ (Fig. 1d). Finally, one obtains

$$
\begin{aligned}
\left\langle\hat{U}_{z_{1}}^{\eta} \otimes \hat{U}_{z_{2}}^{\dagger \eta}\right\rangle_{\text {Fig. } 1}^{\eta_{1}} & \\
= & -\frac{\alpha_{s} \Delta \eta}{\pi^{2}}\left(T^{a} U_{z_{1}} \otimes T^{b} U_{z_{2}}^{\dagger}+U_{z_{1}} T^{b} \otimes U_{z_{2}}^{\dagger} T^{a}\right) \int d^{2} z_{\perp} \frac{\left(z_{13}, z_{23}\right)_{\perp}}{z_{13}^{2} z_{23}^{2}} U_{z_{3}}^{a b} \\
& +\frac{\alpha_{s} \Delta \eta}{\pi^{2}}\left(T^{a} U_{z_{1}} T^{b} \otimes U_{z_{2}}^{\dagger}\right) \int \frac{d^{2} z_{3}}{z_{13}^{2}} U_{z_{3}}^{a b}+\frac{\alpha_{s} \Delta \eta}{\pi^{2}}\left(U_{z_{1}} \otimes T^{b} U_{z_{2}}^{\dagger} T^{a}\right) \int \frac{d^{2} z_{3}}{z_{23}^{2}} U_{z_{3}}^{a b},
\end{aligned}
$$

so

$$
\begin{aligned}
& \left\langle\left.\operatorname{Tr}\left\{\hat{U}_{z_{1}}^{\eta} \hat{U}_{z_{2}}^{\dagger \eta}\right\}\right|_{\text {Fig. } 1} ^{\eta_{1}}\right. \\
& \quad=\frac{\alpha_{s} \Delta \eta}{\pi^{2}} \int d^{2} z_{3} \frac{z_{12}^{2}}{z_{13}^{2} z_{23}^{2}}\left[\operatorname{Tr}\left\{T^{a} U_{z_{1}} U_{z_{3}}^{\dagger} T^{a} U_{z_{3}} U_{z_{2}}^{\dagger}\right\}-\frac{1}{N_{c}} \operatorname{Tr}\left\{U_{z_{1}} U_{z_{2}}^{\dagger}\right\}\right] .
\end{aligned}
$$

There are also contributions coming from the diagrams shown in Fig. 2 (plus graphs obtained by reflection with respect to the shock wave).

These diagrams are proportional to the original dipole $\operatorname{Tr}\left\{U_{z_{1}} U_{z_{2}}^{\dagger}\right\}$ and therefore the corresponding term can be derived from the contribution of Fig. 1 graphs using the requirement that the r.h.s. of the evolution equation should vanish in the absence of the shock wave (when $U \equiv 1$ ). 

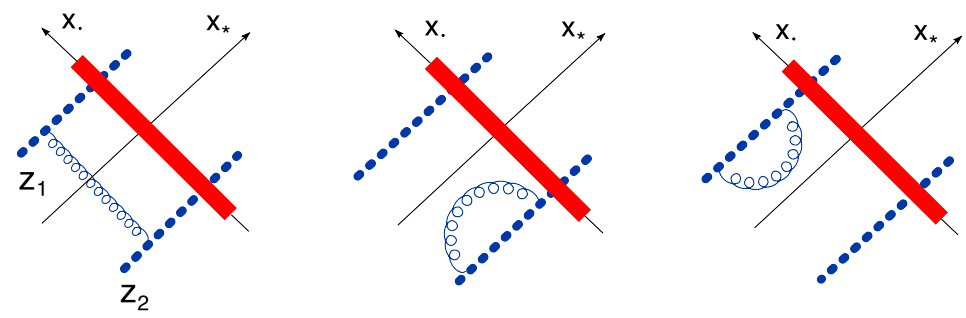

Fig. 2. Leading-order diagrams proportional to the original dipole.

It is easy to see that this requirement leads to

$$
\left\langle\operatorname{Tr}\left\{\hat{U}_{z_{1}}^{\eta_{1}} \hat{U}_{z_{2}}^{\dagger \eta_{1}}\right\}\right\rangle=\frac{\alpha_{s} \Delta \eta}{\pi^{2}} \int d^{2} z_{3} \frac{z_{12}^{2}}{z_{13}^{2} z_{23}^{2}}\left[\operatorname{Tr}\left\{T^{a} U_{z_{1}} U_{z_{3}}^{\dagger} T^{a} U_{z_{3}} U_{z_{2}}^{\dagger}\right\}-N_{c} \operatorname{Tr}\left\{U_{z_{1}} U_{z_{2}}^{\dagger}\right\}\right],
$$

which gives the BK equation for the evolution of the color dipole in the adjoint representation:

$$
\frac{d}{d \eta} \operatorname{Tr}\left\{\hat{U}_{z_{1}}^{\eta} \hat{U}_{z_{2}}^{\dagger \eta}\right\}=\frac{\alpha_{s}}{\pi^{2}} \int d^{2} z_{3} \frac{z_{12}^{2}}{z_{13}^{2} z_{23}^{2}}\left[\operatorname{Tr}\left\{T^{a} U_{z_{1}} U_{z_{3}}^{\dagger} T^{a} U_{z_{3}} U_{z_{2}}^{\dagger}\right\}-N_{c} \operatorname{Tr}\left\{U_{z_{1}} U_{z_{2}}^{\dagger}\right\}\right] .
$$

\section{Calculation of the NLO BK kernel in $\mathcal{N}=4 \mathrm{SYM}$}

In the next-to-leading order the contributions to the kernel come from the one-loop diagrams for the color dipole in the shock-wave background. We will take the results for the gluon part of the NLO BK kernel from Ref. [11] and calculate the contribution of scalar and gluino loops. We use the $\mathcal{N}=4$ Lagrangian in the form (see e.g. Ref. [15]):

$$
\begin{aligned}
\mathcal{L}= & -\frac{1}{4} F^{\mu \nu} F_{\mu \nu}+\frac{1}{2}\left(D^{\mu} \Phi_{I}^{a}\right)\left(D_{\mu} \Phi_{I}^{a}\right)-\frac{1}{4} g^{2} f^{a b c} f^{l m c} \Phi_{I}^{a} \Phi_{J}^{b} \Phi_{I}^{l} \Phi_{J}^{m} \\
& +\bar{\lambda}_{\dot{\alpha} A}^{a} \sigma_{\mu}^{\dot{\alpha} \beta} \mathcal{D}^{\mu} \lambda_{\beta}^{a A}-i \lambda_{a}^{\alpha A} \bar{\Sigma}_{A B}^{s} \Phi_{b}^{s} \lambda_{\alpha k}^{B} f^{a b c}+i \bar{\lambda}_{\dot{\alpha} A}^{a} \Sigma^{s A B} \Phi_{b}^{s} \bar{\lambda}_{B c}^{\dot{\alpha}} f^{a b c} .
\end{aligned}
$$

Here $\Phi_{I}^{a}$ are scalars, $\lambda_{a}^{\alpha A}$ gluinos and $\Sigma_{I J}^{a}=\left(\eta_{A B}^{i}, i \bar{\eta}_{A B}^{i}\right), \bar{\Sigma}_{I J}^{a}=\left(\eta_{A B}^{i},-i \bar{\eta}_{A B}^{i}\right)$ where $\eta_{A B}^{i}$ are standard 't Hooft symbols. The bare propagators are

$$
\begin{aligned}
& \left\langle\Phi_{I}^{a}(x) \Phi_{J}^{b}(y)\right\rangle=i \delta^{a b} \delta_{I J} \int d^{4} p \frac{e^{-i p \cdot(x-y)}}{p^{2}+i \epsilon}, \\
& \left\langle\lambda_{\beta}^{a I}(x) \bar{\lambda}_{\dot{\alpha}}^{b J}(y)\right\rangle=\int d^{4} p e^{-i p \cdot(x-y)} \frac{i p_{\mu} \bar{\sigma}_{\beta \dot{\alpha}}^{\mu}}{p^{2}+i \epsilon},
\end{aligned}
$$

and the vertex of gluon emission in the momentum space is proportional to $\left(k_{1}-k_{2}\right)^{\mu} T^{a} \delta_{I J}$ for the scalars and $\sigma^{\mu} T^{a}$ for gluinos. (We do not need Yukawa or four-scalar vertices at this level.) The diagrams in the shock-wave background are calculated similarly to the tree diagrams discussed in the previous section.

\subsection{Gluon contribution to $N L O B K$}

Let us start with the gluon contribution to the NLO evolution kernel. There is no difference between the gluon part of the kernel in QCD and in $\mathcal{N}=4$ SYM so we will just copy it from Ref. [11] replacing $\operatorname{tr}\left\{t^{a} U_{z_{1}} t^{b} U_{z_{2}}^{\dagger}\right\}$ in the fundamental representation by $\operatorname{Tr}\left\{T^{a} U_{z_{1}} T^{b} U_{z_{2}}^{\dagger}\right\}$ 
(throughout the paper we denote traces in the fundamental and the adjoint representations of color group by $\operatorname{tr}\{\cdots\}$ and $\operatorname{Tr}\{\cdots\}$, respectively)

$$
\begin{aligned}
\frac{d}{d \eta} \operatorname{Tr} & \left\{\hat{U}_{z_{1}}^{\eta} \hat{U}_{z_{2}}^{\dagger \eta}\right\}_{\text {gluon }} \\
= & \frac{\alpha_{s}}{\pi^{2}} \int d^{2} z_{3} \frac{z_{12}^{2}}{z_{13}^{2} z_{23}^{2}}\left\{1+\frac{\alpha_{s} N_{c}}{4 \pi}\left[\frac{11}{3} \ln z_{12}^{2} \mu^{2}-\frac{11}{3} \frac{z_{13}^{2}-z_{23}^{2}}{z_{12}^{2}} \ln \frac{z_{13}^{2}}{z_{23}^{2}}+\frac{64}{9}-\pi^{2} 3\right.\right. \\
& \left.\left.-2 \ln \frac{z_{13}^{2}}{z_{12}^{2}} \ln \frac{z_{23}^{2}}{z_{12}^{2}}\right]\right\}\left[\operatorname{Tr}\left\{T^{a} \hat{U}_{z_{1}}^{\eta} \hat{U}_{z_{3}}^{\dagger \eta} T^{a} \hat{U}_{z_{3}} \hat{U}_{z_{2}}^{\dagger \eta}\right\}-N_{c} \operatorname{Tr}\left\{\hat{U}_{z_{1}}^{\eta} \hat{U}_{z_{2}}^{\dagger \eta}\right\}\right] \\
+ & \frac{\alpha_{s}^{2}}{8 \pi^{4}} \int \frac{d^{2} z_{3} d^{2} z_{4}}{z_{34}^{4}}\left(\hat{U}_{z_{3}}^{\eta}\right){ }^{a a^{\prime}}\left[\left\{2\left[2-\frac{z_{13}^{2} z_{24}^{2}+z_{14}^{2} z_{23}^{2}-4 z_{12}^{2} z_{34}^{2}}{z_{13}^{2} z_{24}^{2}-z_{14}^{2} z_{23}^{2}} \ln \frac{z_{13}^{2} z_{24}^{2}}{z_{14}^{2} z_{23}^{2}}\right]\right.\right. \\
& \times \operatorname{Tr}\left\{\left[T^{a}, T^{b}\right] U_{z_{1}}\left[T^{a^{\prime}}, T^{b^{\prime}}\right] U_{z_{2}}^{\dagger}\right\} \\
& -\left[\frac{z_{12}^{2} z_{34}^{2}}{z_{13}^{2} z_{24}^{2}}\left(1+\frac{z_{12}^{2} z_{34}^{2}}{z_{13}^{2} z_{24}^{2}-z_{23}^{2} z_{14}^{2}}\right) \ln \frac{z_{13}^{2} z_{24}^{2}}{z_{14}^{2} z_{23}^{2}}+z_{3} \leftrightarrow z_{4}\right] \\
& \left.\times \operatorname{Tr}\left\{\left[T^{a}, T^{b}\right] U_{z_{1}} T^{a^{\prime}} T^{b^{\prime}} U_{z_{2}}^{\dagger}+T^{b} T^{a} U_{z_{1}}\left[T^{b^{\prime}}, T^{a^{\prime}}\right] U_{z_{2}}^{\dagger}\right\}\right\}\left(\hat{U}_{z_{4}}^{\eta}-\hat{U}_{z_{3}}^{\eta}\right)^{b b^{\prime}} \\
& -\left[\frac{z_{12}^{2} z_{34}^{2}}{z_{13}^{2} z_{24}^{2}}\left(1+\frac{z_{12}^{2} z_{34}^{2}}{z_{13}^{2} z_{24}^{2}-z_{23}^{2} z_{14}^{2}}\right) \ln \frac{z_{13}^{2} z_{24}^{2}}{z_{14}^{2} z_{23}^{2}}-z_{3} \leftrightarrow z_{4}\right] \\
& \left.\times \operatorname{Tr}\left\{\left[T^{a}, T^{b}\right] U_{z_{1}} T^{a^{\prime}} T^{b^{\prime}} U_{z_{2}}^{\dagger}+T^{b} T^{a} U_{z_{1}}\left[T^{b^{\prime}}, T^{a^{\prime}}\right] U_{z_{2}}^{\dagger}\right\} U_{z_{4}}^{b b^{\prime}}\right],
\end{aligned}
$$

where $\mu$ is the normalization point in the $\overline{\mathrm{MS}}$ scheme. Our normalization fro Gell-Mann matrices is $\operatorname{tr}\left\{t^{a} t^{b}\right\}=\frac{1}{2} \delta^{a b}$.

Note that the last term in r.h.s. is Möbius-invariant. The coefficient $\frac{11}{3}$ stands in front of the non-conformal terms coming from the running of the coupling constant and as discussed in the Introduction, there is an additional non-conformal term $\sim \ln \frac{z_{12}^{2}}{z_{13}^{2}} \ln \frac{z_{12}^{2}}{z_{23}^{2}}$ coming from the non-invariance of the longitudinal cutoff (8).

It should be noted also that there is one small difference between QCD and $\mathcal{N}=4$ calculations of the gluon loop due to the fact that in supersymmetric theories it is more natural to use the dimensional reduction scheme [16] instead of dimensional regularization. In dimensional reduction scheme the factor $g_{\mu \nu}^{\perp} g^{\perp \mu \nu}=d_{\perp}$ coming from the product of three-gluon vertices should be replaced by 2: $g_{\mu \nu}^{\perp} g^{\perp \mu \nu} \rightarrow 2$. Making proper replacement in formulas in Section IV of Ref. [11] one gets the factor $\frac{64}{9}$ in the r.h.s. of the above equation in place of $\frac{67}{9}$ in Eq. (5) in Ref. [11].

\subsection{Contribution of scalar particles}

\subsubsection{Diagrams with two scalar-shockwave intersections}

First, we calculate the diagram with two scalar-shockwave intersections shown in Fig. 3. The scalar propagator in the shock-wave background has the form [2]: 

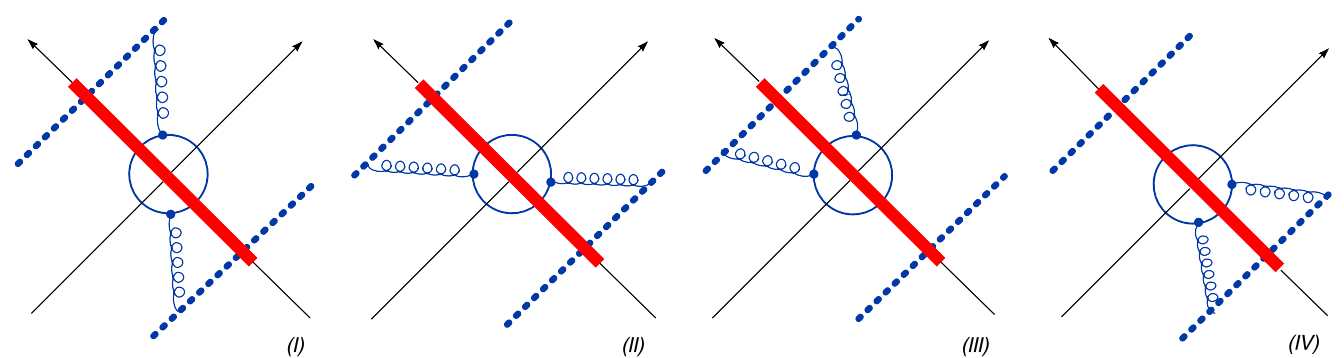

(III)

(IV)

Fig. 3. Diagrams with the scalar loop bisected by the shock wave.

$$
\begin{aligned}
\left\langle\hat{\Phi}_{I}^{a}(x) \hat{\Phi}_{J}^{b}(y)\right\rangle= & \theta\left(x_{*} y_{*}\right) \delta^{a b} \frac{s}{2} \int d \alpha d \beta\left(x_{\perp}\left|\frac{i \delta_{I J}}{i\left(\alpha \beta s-p_{\perp}^{2}+i \epsilon\right)}\right| y_{\perp}\right) \\
& +\delta_{I J} \int_{0}^{\infty} d \alpha \frac{e^{-i \alpha(x-y)}}{2 \alpha}\left[\theta ( x _ { * } ) \theta ( - y _ { * } ) \left(x_{\perp} \mid e^{-i \frac{p_{\perp}^{2}}{\alpha s} x_{*}} U^{a b} e^{\left.i \frac{p_{\perp}^{2}}{\alpha s} y_{*} \mid y_{\perp}\right)}\right.\right. \\
& \left.+\theta\left(-x_{*}\right) \theta\left(y_{*}\right)\left(x_{\perp}\left|e^{i \frac{p_{\perp}^{2}}{\alpha s} x_{*}} U^{\dagger a b} e^{-i \frac{p_{\perp}^{2}}{\alpha s} y_{*}}\right| y_{\perp}\right)\right] .
\end{aligned}
$$

We start with the calculation of the Fig. 3a diagram. Multiplying two propagators (22), two scalar-gluon vertices and two bare gluon propagators we get

$$
\begin{aligned}
\left\langle\hat{U}_{x}^{\eta} \hat{U}_{y}^{\dagger \eta}\right\rangle_{\text {Fig. 3a }} & g^{2} \int_{0}^{\infty} d u \int_{-\infty}^{0} d v\left\langle\hat{A}_{\bullet}^{a}\left(u p_{1}+z_{1}\right) \hat{A}_{\bullet}^{b}\left(v p_{1}+z_{2}\right)\right\rangle \\
= & -6 g^{4} \frac{s^{2}}{8} f^{a n l} f^{b n^{\prime} l^{\prime}} \int d \alpha d \alpha_{1} d \beta d \beta^{\prime} d \beta_{1} d \beta_{1}^{\prime} d \beta_{2} d \beta_{2}^{\prime} \int d^{2} z d^{2} z_{4} \\
& \times \int d^{2} q_{1} d^{2} q_{2} d^{2} k_{1} d^{2} k_{2} e^{i\left(q_{1}+q_{2}, z_{1}\right)_{\perp}-i\left(k_{1}+k_{2}, z_{2}\right)_{\perp}} \\
& \times \frac{4 \alpha_{1}\left(\alpha-\alpha_{1}\right) U_{z_{3}}^{n n^{\prime}} U_{z_{4}}^{l l^{\prime}} e^{-i\left(q_{1}-k_{1}, z_{3}\right)_{\perp}-i\left(q_{2}-k_{2}, z_{4}\right)_{\perp}}}{\left(\beta-\beta_{1}-\beta_{2}+i \epsilon\right)\left(\beta^{\prime}-\beta_{1}^{\prime}-\beta_{2}^{\prime}+i \epsilon\right)(\beta-i \epsilon)\left(\beta^{\prime}-i \epsilon\right)} \\
& \times \frac{d_{\bullet \lambda}\left(\alpha p_{1}+\beta p_{2}+q_{1 \perp}+k_{1 \perp}\right)}{\alpha \beta s-\left(q_{1}+q_{2}\right)_{\perp}^{2}+i \epsilon} \frac{d_{\lambda^{\prime}}\left(\alpha p_{1}+\beta^{\prime} p_{2}+q_{2 \perp}+k_{2 \perp}\right)}{\alpha \beta^{\prime} s-\left(k_{1}+k_{2}\right)_{\perp}^{2}+i \epsilon} \\
& \times \frac{\left[\left(2 \alpha_{1}-\alpha\right) p_{1}+\left(q_{1}-q_{2}\right) \perp\right]^{\lambda}\left[\left(2 \alpha_{1}-\alpha\right) p_{1}+\left(k_{1}-k_{2}\right)_{\perp}\right]^{\lambda^{\prime}}}{\left(\alpha_{1} \beta_{1} s-q_{1 \perp}^{2}+i \epsilon\right)\left(\alpha_{1} \beta_{1}^{\prime} s-k_{1 \perp}^{2}+i \epsilon\right)\left[\left(\alpha-\alpha_{1}\right) \beta_{2} s-q_{2 \perp}^{2}+i \epsilon\right]\left[\left(\alpha-\alpha_{1}\right) \beta_{2}^{\prime} s-k_{2 \perp}^{2}+i \epsilon\right]} .
\end{aligned}
$$

Taking residues at $\beta=\beta^{\prime}=0$ and $\beta_{2}=-\beta_{1}, \beta_{2}^{\prime}=-\beta_{1}^{\prime}$ we obtain

$$
\begin{aligned}
& g^{2} \int_{0}^{\infty} d u \int_{-\infty}^{0} d v\left\langle\hat{A}_{\bullet}^{a}\left(u p_{1}+z_{1}\right) \hat{A}_{\bullet}^{b}\left(v p_{1}+z_{2}\right)\right\rangle \\
& \quad=-6 g^{4} \frac{s^{2}}{8} f^{a n l} f^{b n^{\prime} l^{\prime}} \int d \alpha d \alpha_{1} d \beta_{1} d \beta_{1}^{\prime} \int d^{2} z d^{2} z_{4}
\end{aligned}
$$




$$
\begin{aligned}
& \times \int d^{2} q_{1} d^{2} q_{2} d^{2} k_{1} d^{2} k_{2} e^{i\left(q_{1}+q_{2}, z_{1}\right) \perp-i\left(k_{1}+k_{2}, z_{2}\right)_{\perp}} \\
& \times 4 \frac{\alpha_{1}\left(\alpha-\alpha_{1}\right)}{\alpha^{2}} U_{z_{3}}^{n n^{\prime}} U_{z_{4}}^{l l^{\prime}} e^{-i\left(q_{1}-k_{1}\right) z-i\left(q_{2}-k_{2}\right) z_{4}} \frac{q_{1 \perp}^{2}-q_{2 \perp}^{2}}{\left(q_{1}+q_{2}\right)_{\perp}^{2}} \frac{k_{1 \perp}^{2}-k_{2 \perp}^{2}}{\left(k_{1}+k_{2}\right)_{\perp}^{2}} \\
& \times \frac{1}{\left(\alpha_{1} \beta_{1} s-q_{1 \perp}^{2}+i \epsilon\right)\left(\alpha_{1} \beta_{1}^{\prime} s-k_{1 \perp}^{2}+i \epsilon\right)\left[-\left(\alpha-\alpha_{1}\right) \beta_{1} s-q_{2 \perp}^{2}+i \epsilon\right]\left[-\left(\alpha-\alpha_{1}\right) \beta_{1}^{\prime} s-k_{2 \perp}^{2}+i \epsilon\right]} .
\end{aligned}
$$

Finally, we can take residues at $\beta_{1}=\frac{q_{1}^{2}}{\alpha_{1} s}$ and $\beta_{1}^{\prime}=\frac{k_{1}^{2}}{\alpha_{1} s}$ :

$$
\begin{aligned}
\left\langle\hat{U}_{x}^{\eta} \hat{U}_{y}^{\dagger \eta}\right\rangle_{\text {Fig. 3a }} & \\
= & -6 \frac{g^{4}}{8 \pi^{2}} f^{a n l} f^{b n^{\prime} l^{\prime}} \int_{0}^{\sigma} \frac{d \alpha}{\alpha} \int_{0}^{1} d u \bar{u} u \int d^{2} z d^{2} z_{4} \\
& \times \int d^{2} q_{1} d^{2} q_{2} d^{2} k_{1} d^{2} k_{2} e^{i\left(q_{1}+q_{2}, z_{1}\right)_{\perp}-i\left(k_{1}+k_{2}, z_{2}\right)_{\perp}} e^{-i\left(q_{1}-k_{1}, z_{3}\right)_{\perp}-i\left(q_{2}-k_{2}, z_{4}\right)_{\perp}} \\
& \times \frac{\left(q_{1 \perp}^{2}-q_{2 \perp}^{2}\right)\left(k_{1 \perp}^{2}-k_{2 \perp}^{2}\right)}{\left(q_{1}+q_{2}\right)_{\perp}^{2}\left(k_{1}+k_{2}\right)_{\perp}^{2}} \frac{1}{\left(q_{1 \perp}^{2} \bar{u}+q_{2 \perp}^{2} u\right)\left(k_{1 \perp}^{2} \bar{u}+k_{2 \perp}^{2} u\right)} U_{z_{3}}^{n n^{\prime}} U_{z_{4}}^{l l^{\prime}},
\end{aligned}
$$

where $u=\alpha_{1} / \alpha$ and $\bar{u} \equiv 1-u$. The contribution of the diagram in Fig. $3 \mathrm{~b}$ is obtained by replacing $e^{i\left(q_{1}+q_{2}, z_{1}\right) \perp}$ by $-e^{i\left(q_{1}+q_{2}, z_{2}\right) \perp}$ and the two remaining diagrams in Fig. $3 \mathrm{c}$ and Fig. $3 \mathrm{~d}$ are obtained by change $x \leftrightarrow y$. We get

$$
\begin{aligned}
\left\langle\operatorname{Tr}\left\{\hat{U}_{z_{1}}^{\eta} \hat{U}_{z_{2}}^{\dagger}\right\}\right\rangle_{\text {Fig. } 3} \\
=6 \frac{g^{4}}{8 \pi^{2}} f^{a n l} f^{b n^{\prime} l^{\prime}} \int_{0}^{\sigma} \frac{d \alpha}{\alpha} \int_{0}^{1} d u \bar{u} u \int d^{2} z d^{2} z_{4} \\
\quad \times \int d^{2} q_{1} d^{2} q_{2} d^{2} k_{1} d^{2} k_{2}\left[e^{i\left(q_{1}+q_{2}, z_{1}\right)_{\perp}}-e^{i\left(q_{1}+q_{2}, z_{2}\right)_{\perp}}\right] \\
\quad \times\left[e^{i\left(k_{1}+k_{2}, z_{1}\right)_{\perp}}-e^{-i\left(k_{1}+k_{2}, z_{2}\right) \perp}\right] \\
\quad \times \frac{\left(q_{1 \perp}^{2}-q_{2 \perp}^{2}\right)\left(k_{1 \perp}^{2}-k_{2 \perp}^{2}\right)}{\left(q_{1}+q_{2}\right)_{\perp}^{2}\left(k_{1}+k_{2}\right)_{\perp}^{2}} \frac{e^{-i\left(q_{1}-k_{1}, z_{3}\right)-i\left(q_{2}-k_{2}, z_{4}\right)}}{\left(q_{1 \perp}^{2} \bar{u}+q_{2 \perp}^{2} u\right)\left(k_{1 \perp}^{2} \bar{u}+k_{2 \perp}^{2} u\right)} U_{z_{3}}^{n n^{\prime}} U_{z_{4}}^{l l^{\prime}} .
\end{aligned}
$$

Performing the Fourier transformation

$$
\begin{aligned}
& \int d^{2} q_{1} d^{2} q_{2} e^{i\left(q_{1}, x_{1}\right)+i\left(q_{2}, x_{2}\right)} \frac{q_{1}^{2}-q_{2}^{2}}{\left(q_{1}+q_{2}\right)^{2}\left(q_{1}^{2} \bar{u}+q_{2}^{2} u\right)} \\
& =-\frac{x_{1}^{2}-x_{2}^{2}}{4 \pi^{2}\left(x_{1}-x_{2}\right)^{2}\left(u x_{1}^{2}+\bar{u} x_{2}^{2}\right)}
\end{aligned}
$$

we obtain

$$
\begin{aligned}
& \left\langle\operatorname{Tr}\left\{\hat{U}_{z_{1}}^{\eta} \hat{U}_{z_{2}}^{\dagger \eta}\right\}\right\rangle_{\text {Fig. } 3} \\
& \quad=6 \frac{g^{4}}{128 \pi^{6}} f^{a n l} f^{b n^{\prime} l^{\prime}} \int_{0}^{\sigma} \frac{d \alpha}{\alpha} \int_{0}^{1} d u \int d^{2} z d^{2} z_{4} U^{n n^{\prime}}(z) U^{l l^{\prime}}\left(z_{4}\right) \frac{\bar{u} u}{z_{34}^{4}}
\end{aligned}
$$




$$
\begin{aligned}
& \times\left[\frac{z_{13}^{2}-z_{14}^{2}}{z_{13}^{2} u+z_{14}^{2} \bar{u}}-\frac{z_{23}^{2}-z_{24}^{2}}{z_{23}^{2} u+z_{24}^{2} \bar{u}}\right]\left[\frac{z_{13}^{2}-z_{14}^{2}}{z_{13}^{2} u+z_{14}^{2} \bar{u}}-\frac{z_{23}^{2}-z_{24}^{2}}{z_{23}^{2} u+z_{24}^{2} \bar{u}}\right] \\
& \times \operatorname{Tr}\left\{T^{a} U_{z_{1}} T^{b} U_{z_{2}}^{\dagger}\right\},
\end{aligned}
$$

and therefore

$$
\begin{aligned}
\sigma \frac{d}{d \sigma} & \left.\operatorname{Tr}\left\{\hat{U}_{z_{1}}^{\eta} \hat{U}_{z_{2}}^{\dagger \eta}\right\}\right\rangle_{\text {Fig. } 3} \\
= & -\frac{3 \alpha^{2}}{4 \pi^{4}} \int \frac{d^{2} z_{3} d^{2} z_{4}}{z_{34}^{4}}\left[-2+\frac{z_{13}^{2} z_{24}^{2}+z_{14}^{2} z_{23}^{2}}{z_{13}^{2} z_{24}^{2}-z_{14}^{2} z_{23}^{2}} \ln \frac{z_{13}^{2} z_{24}^{2}}{z_{14}^{2} z_{23}^{2}}\right] \\
& \times U_{z_{3}}^{a a^{\prime}} U_{z_{4}}^{b b^{\prime}} \operatorname{Tr}\left\{\left[T^{a}, T^{b}\right] U_{z_{1}}\left[T^{a^{\prime}}, T^{b^{\prime}}\right] U_{z_{2}}^{\dagger}\right\} .
\end{aligned}
$$

Following the method suggested in Refs. [11,17,18] we separate the UV-divergent part by adding and subtracting $z_{4} \rightarrow z_{3}$ contribution: $U_{z_{3}}^{a a^{\prime}} U_{z_{4}}^{b b^{\prime}}=\left(U_{z_{3}}^{a a^{\prime}} U_{z_{4}}^{b b^{\prime}}-z_{4} \rightarrow z_{3}\right)+U_{z_{3}}^{a a^{\prime}} U_{z_{3}}^{b b^{\prime}}$. We get

$$
\begin{aligned}
\sigma \frac{d}{d \sigma}\left\langle\operatorname{Tr}\left\{\hat{U}_{z_{1}}^{\eta} \hat{U}_{z_{2}}^{\dagger \eta}\right\}\right\rangle_{\text {Fig. } 3}= & -\frac{3 \alpha^{2}}{4 \pi^{4}} \int \frac{d^{2} z_{3} d^{2} z_{4}}{z_{34}^{4}}\left[-2+\frac{z_{13}^{2} z_{24}^{2}+z_{14}^{2} z_{23}^{2}}{z_{13}^{2} z_{24}^{2}-z_{14}^{2} z_{23}^{2}} \ln \frac{z_{13}^{2} z_{24}^{2}}{z_{14}^{2} z_{23}^{2}}\right] \\
& \times\left(U_{z_{3}}^{a a^{\prime}} U_{z_{4}}^{b b^{\prime}}-z_{4} \rightarrow z_{3}\right) \operatorname{Tr}\left\{\left[T^{a}, T^{b}\right] U_{z_{1}}\left[T^{a^{\prime}}, T^{b^{\prime}}\right] U_{z_{2}}^{\dagger}\right\} \\
& +\frac{3 \alpha^{2} N_{c}}{4 \pi^{4}} \int d^{2} z \operatorname{Tr}\left\{T^{a} U_{z_{1}} U_{z_{3}}^{\dagger} T^{a} U_{z_{3}} U_{z_{2}}^{\dagger}\right\} \\
& \times \int \frac{d^{2} z_{4}}{z_{34}^{4}}\left[-2+\frac{z_{13}^{2} z_{24}^{2}+z_{14}^{2} z_{23}^{2}}{z_{13}^{2} z_{24}^{2}-z_{14}^{2} z_{23}^{2}} \ln \frac{z_{13}^{2} z_{24}^{2}}{z_{14}^{2} z_{23}^{2}}\right] .
\end{aligned}
$$

The second (UV-divergent) part should be calculated at $d_{\perp} \neq 2$. As in the case of gluon loop, the Fourier transform (27) at $d_{\perp} \neq 2$ is complicated so it is convenient to return to Eq. (26) in the momentum representation. After replacing $U_{z_{3}}^{m m^{\prime}} U_{z_{4}}^{n n^{\prime}}$ by $U_{z_{3}}^{m m^{\prime}} U_{z_{3}}^{n n^{\prime}}$, integrating over $u$ and changing variables to $k_{2}=q_{2}=k^{\prime}, p=q_{1}+q_{2}, l=q_{1}-k_{1}$ (so that $q_{1}=p-k^{\prime}, k_{1}=p-l-k^{\prime}$ and $k_{1}+k_{2}=p-l$ ) Eq. (26) turns into

$$
\begin{aligned}
\left\langle\operatorname{Tr}\left\{\hat{U}_{z_{1}}^{\eta} \hat{U}_{z_{2}}^{\dagger \eta}\right\}\right\rangle_{\mathrm{Fig} .3 z_{4} \rightarrow z_{3}} \\
=\frac{3 g^{4} N_{c}}{4 \pi^{2}} \int_{0}^{\sigma} \frac{d \alpha}{\alpha} \int d^{2} z_{3} \operatorname{Tr}\left\{T^{a} U_{z_{1}} U_{z_{3}}^{\dagger} T^{a} U_{z_{3}} U_{z_{2}}^{\dagger}\right\} \\
\quad \times \int d^{2-\varepsilon} p{d^{2-\varepsilon}}^{2-}\left(e^{i\left(p, z_{13}\right)}-e^{i\left(p, z_{23}\right)}\right)\left(e^{-i\left(p-l, z_{13}\right)}-e^{-i\left(p-l, z_{23}\right)}\right) \Phi(p, l),
\end{aligned}
$$

where

$$
\begin{aligned}
\Phi(p, l)= & \mu^{2 \epsilon} \int d^{2-\varepsilon} k^{\prime} \frac{1}{p^{2}(p-l)^{2}}\left[-2-\frac{\left(p-k^{\prime}\right)^{2}+\left(p-k^{\prime}-l\right)^{2}}{\left(p-k^{\prime}\right)^{2}-\left(p-k^{\prime}-l\right)^{2}} \ln \frac{\left(p-k^{\prime}\right)^{2}}{\left(p-k^{\prime}-l\right)^{2}}\right. \\
& \left.+\frac{k^{\prime 2}+\left(p-k^{\prime}\right)^{2}}{\left(p-k^{\prime}\right)^{2}-k^{\prime 2}} \ln \frac{\left(p-k^{\prime}\right)^{2}}{k^{\prime 2}}+\frac{\left(p-k^{\prime}-l\right)^{2}+k^{\prime 2}}{\left(p-k^{\prime}-l\right)^{2}-k^{\prime 2}} \ln \frac{\left(p-k^{\prime}-l\right)^{2}}{k^{\prime 2}}\right] \\
= & \frac{\Gamma^{2}\left(1-\frac{\epsilon}{2}\right) \Gamma\left(\frac{\epsilon}{2}\right)}{4 \pi(3-\epsilon) \Gamma(2-\epsilon)} \frac{1}{p^{2}(p-l)^{2}}\left(p^{2-\epsilon}+|p-l|^{2-\epsilon}-l^{2-\epsilon}\right)
\end{aligned}
$$




$$
\begin{aligned}
= & \frac{(p, p-l)}{2 \pi p^{2}(p-l)^{2}}\left(\frac{2}{\epsilon}-\ln \frac{l^{2}}{\mu^{2}}+\frac{8}{3}\right)-\frac{\ln p^{2} / l^{2}}{3(p-l)^{2}}-\ln (p-l)^{2} / l^{2} 3 p^{2} \\
& +O(\epsilon) .
\end{aligned}
$$

Subtracting the pole in $\epsilon$ corresponding to counterterm (see the discussion in Refs. $[11,17]$ ) we obtain

$$
\begin{aligned}
\sigma \frac{d}{d \sigma} & \left\langle\operatorname{Tr}\left\{\hat{U}_{z_{1}}^{\eta} \hat{U}_{z_{2}}^{\dagger \dagger}\right\}\right\rangle_{\mathrm{Fig} .3 z_{4} \rightarrow z_{3}} \\
= & 3 \frac{\alpha_{s}^{2}}{\pi} N_{c} \int d^{2} z \operatorname{Tr}\left\{T^{a} U_{z_{1}} U_{z_{3}}^{\dagger} T^{b} U_{z_{3}} U_{z_{2}}^{\dagger}\right\} \\
& \times \int d^{2-\varepsilon} p d^{2-\varepsilon} l\left(e^{i\left(p, z_{13}\right)}-e^{i\left(p, z_{23}\right)}\right)\left(e^{-i\left(p-l, z_{13}\right)}-e^{-i\left(p-l, z_{23}\right)}\right) \\
& \times\left[\frac{2(p, p-l)}{3 p^{2}(p-l)^{2}}\left(-\ln \frac{l^{2}}{\mu^{2}}+\frac{8}{3}\right)-\frac{\ln p^{2} / l^{2}}{3(p-l)^{2}}-\frac{\ln (p-l)^{2} / l^{2}}{3 p^{2}}\right] .
\end{aligned}
$$

Using the Fourier integral from Appendix A to [11] we get

$$
\begin{aligned}
\int d^{2} p d^{2} l e^{i\left(p, z_{13}\right)-i\left(p-l, z_{23}\right)}\left[\frac{2(p, p-l)}{3 p^{2}(p-l)^{2}}\left(-\ln \frac{l^{2}}{\mu^{2}}+\frac{8}{3}\right)\right. \\
\left.\quad-\frac{\ln p^{2} / l^{2}}{3(p-l)^{2}}-\frac{\ln (p-l)^{2} / l^{2}}{3 p^{2}}\right] \\
=\frac{\left(z_{13}, z_{23}\right)}{12 \pi^{2} z_{13}^{2} z_{23}^{2}}\left[2 \ln \frac{z_{13}^{2} z_{23}^{2} \mu^{2}}{z_{12}^{2}}+\frac{16}{3}\right]-\frac{1}{12 \pi^{2} z_{23}^{2}} \ln \frac{z_{13}^{2}}{z_{12}^{2}}-\frac{1}{12 \pi^{2} z_{13}^{2}} \ln \frac{z_{23}^{2}}{z_{12}^{2}} \\
=-\frac{z_{12}^{2}}{12 \pi^{2} z_{13}^{2} z_{23}^{2}}\left[\ln \frac{z_{13}^{2} z_{23}^{2} \mu^{2}}{z_{12}^{2}}+\frac{8}{3}\right]+\frac{1}{12 \pi^{2} z_{13}^{2}} \ln z_{13}^{2} \mu^{2}+\frac{1}{12 \pi^{2} z_{23}^{2}} \ln z_{23}^{2} \mu^{2},
\end{aligned}
$$

and therefore

$$
\begin{aligned}
& \sigma \frac{d}{d \sigma}\left\langle\operatorname{Tr}\left\{\hat{U}_{z_{1}}^{\eta} \hat{U}_{z_{2}}^{\dagger \eta}\right\}\right\rangle_{\text {Fig. } 3 z_{4} \rightarrow z_{3}} \\
& \quad=\frac{\alpha_{s}^{2} N_{c}}{2 \pi^{3}} \int d^{2} z_{3} \frac{z_{12}^{2}}{z_{13}^{2} z_{23}^{2}}\left[\ln \frac{z_{13}^{2} z_{23}^{2} \mu^{2}}{z_{12}^{2}}+\frac{8}{3}\right] \operatorname{Tr}\left\{T^{a} U_{z_{1}} U_{z_{3}}^{\dagger} T^{b} U_{z_{3}} U_{z_{2}}^{\dagger}\right\} .
\end{aligned}
$$

Combining Eqs. (30) and (35) we obtain the full contribution of diagrams in Fig. 3 to the NLO kernel in the form

$$
\begin{aligned}
\frac{d}{d \eta}( & \left.\operatorname{Tr}\left\{\hat{U}_{z_{1}}^{\eta} \hat{U}_{z_{2}}^{\dagger \eta}\right\}\right)_{\text {scalars }} \\
= & -\frac{3 \alpha^{2}}{4 \pi^{4}} \int \frac{d^{2} z_{3} d^{2} z_{4}}{z_{34}^{4}}\left[-2+\frac{z_{13}^{2} z_{24}^{2}+z_{14}^{2} z_{23}^{2}}{z_{13}^{2} z_{24}^{2}-z_{14}^{2} z_{23}^{2}} \ln \frac{z_{13}^{2} z_{24}^{2}}{z_{14}^{2} z_{23}^{2}}\right] \\
& \times\left(\hat{U}_{z_{3}}^{a a^{\prime}} \hat{U}_{z_{4}}^{b b^{\prime}}-z_{4} \rightarrow z_{3}\right) \operatorname{Tr}\left\{\left[T^{a}, T^{b}\right] \hat{U}_{z_{1}}^{\eta}\left[T^{a^{\prime}}, T^{b^{\prime}}\right] \hat{U}_{z_{2}}\right\} \\
& +\frac{\alpha_{s}^{2} N_{c}}{2 \pi^{3}} \int d^{2} z \frac{z_{12}^{2}}{z_{13}^{2} z_{23}^{2}}\left[\ln \frac{z_{13}^{2} z_{23}^{2} \mu^{2}}{z_{12}^{2}}+\frac{8}{3}\right] \operatorname{Tr}\left\{T^{a} \hat{U}_{z_{1}}^{\eta} \hat{U}_{z_{3}}^{\dagger \eta} T^{a} \hat{U}_{z_{3}} \hat{U}_{z_{2}}^{\dagger \eta}\right\}
\end{aligned}
$$

where we have promoted the shock-wave Wilson lines to operators. 

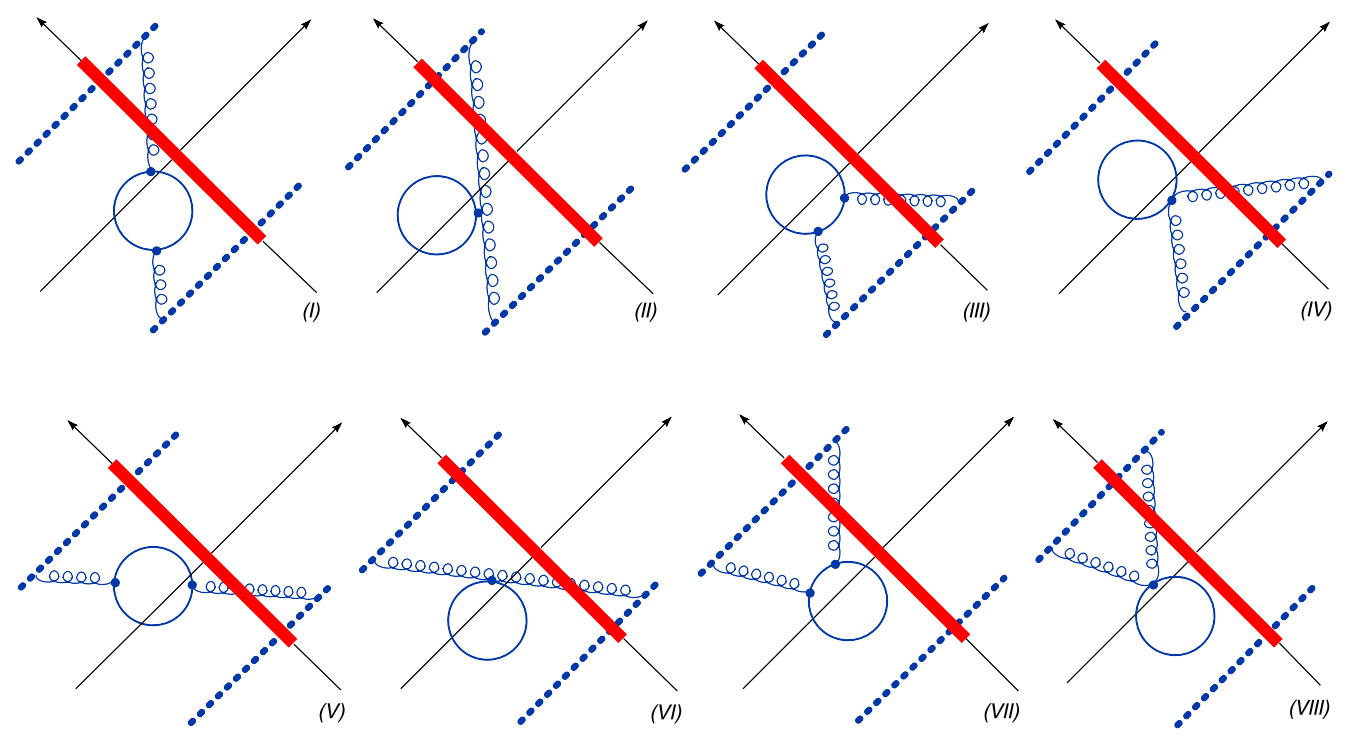

Fig. 4. Diagrams with bare scalar loop.

\subsubsection{Scalar loop}

Besides diagrams with the scalar loop bisected by the shock wave calculated above, there are diagrams with the ordinary scalar loop shown in Fig. 4.

The integral for the scalar loop has the form

$$
\begin{aligned}
\int d^{4-\epsilon} k^{\prime} \frac{\left(2 k^{\prime}-k\right)_{\mu}\left(2 k^{\prime}-k\right)_{\nu}}{\left(k^{\prime 2}+i \epsilon\right)\left[\left(k-k^{\prime}\right)^{2}+i \epsilon\right]} & =\frac{2 i \Gamma\left(\frac{\epsilon}{2}\right) \Gamma\left(1-\frac{\epsilon}{2}\right) \Gamma\left(2-\frac{\epsilon}{2}\right)}{(4 \pi)^{2-\frac{\epsilon}{2}} \Gamma(4-\epsilon)\left(-k^{2}\right)^{\frac{\epsilon}{2}}}\left(k_{\mu} k_{\nu}-g_{\mu \nu}\right) \\
& \simeq \frac{i}{48 \pi^{2}}\left[\frac{2}{\epsilon}+\ln \frac{1}{-k^{2}}+\frac{8}{3}\right]\left(k_{\mu} k_{\nu}-\delta_{\mu \nu}\right)
\end{aligned}
$$

and therefore the contribution of the diagram shown in Fig. 4 takes the form

$$
\begin{aligned}
\frac{d}{d \eta}\left\langle\operatorname{Tr}\left\{\hat{U}_{z_{1}}^{\eta} \hat{U}_{z_{2}}^{\dagger \eta}\right\}\right\rangle_{\text {Fig. } 4} \\
=-\frac{\alpha_{s}^{2} N_{c}}{\pi} \int d^{2} z_{3}\left[\operatorname{Tr}\left\{T^{a} U_{z_{1}} U_{z_{3}}^{\dagger} T^{a} U_{z_{3}} U_{z_{2}}^{\dagger}\right\}-\frac{1}{N_{c}} \operatorname{Tr}\left\{U_{z_{1}} U_{z_{2}}^{\dagger}\right\}\right] \\
\quad \times \int d^{2} k d^{2} k^{\prime} d^{2} q\left[e^{i\left(q, z_{13}\right)}-e^{i\left(q, z_{23}\right)}\right]\left[e^{-i\left(k, z_{13}\right)}-e^{-i\left(k, z_{23}\right)}\right] \\
\quad \times \frac{(q, k)}{k^{2} q^{2}}\left\{\ln \frac{\mu^{2}}{k^{2}}+\frac{8}{3}\right\} .
\end{aligned}
$$

As usual, we should add diagrams obtained by the reflection of diagrams shown in Fig. 4 with respect to the shock-wave line. Their contribution is obtained from Eq. (38) by the replacement $q \leftrightarrow k$ in the logarithm so the final result for the sum of all diagrams of Fig. 4 type has the form 

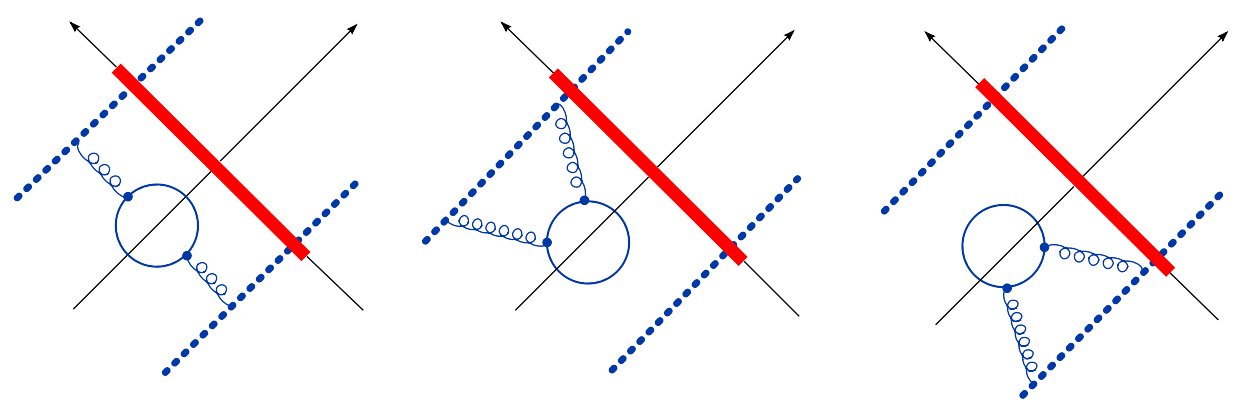

Fig. 5. Diagrams without scalar-shockwave intersection.

$$
\begin{aligned}
\frac{d}{d \eta}\langle\operatorname{Tr} & \left.\left\{\hat{U}_{z_{1}}^{\eta} \hat{U}_{z_{2}}^{\dagger \eta}\right\}\right\rangle_{\text {Fig. } 4+\text { refl. }} \\
= & -\frac{\alpha_{s}^{2} N_{c}}{\pi} \int d^{2} z_{3} \operatorname{Tr}\left\{T^{a} U_{z_{1}} U_{z_{3}}^{\dagger} T^{a} U_{z_{3}} U_{z_{2}}^{\dagger}\right\} \\
& \times \int d^{2} k d^{2} k^{\prime} d^{2} q\left[e^{i\left(q, z_{13}\right)}-e^{i\left(q, z_{23}\right)}\right]\left[e^{-i\left(k, z_{13}\right)}-e^{i\left(k, z_{23}\right)}\right] \\
& \times \frac{(q, k)}{k^{2} q^{2}}\left\{\ln \frac{\mu^{4}}{q^{2} k^{2}}+\frac{16}{3}\right\} \\
= & -\frac{\alpha_{s}^{2} N_{c}}{4 \pi^{3}} \int d^{2} z_{3} \operatorname{Tr}\left\{T^{a} \hat{U}_{z_{1}}^{\eta} \hat{U}_{z_{3}}^{\dagger \eta} T^{a} \hat{U}_{z_{3}} \hat{U}_{z_{2}}^{\dagger \eta}\right\} \\
& \times\left\{\frac{z_{12}^{2}}{z_{13}^{2} z_{23}^{2}}\left[\ln \frac{z_{13}^{2} z_{23}^{2}}{\mu^{-4}}+\frac{16}{3}\right]+\left[\frac{1}{z_{13}^{2}}-\frac{1}{z_{23}^{2}}\right] \ln \frac{z_{13}^{2}}{z_{23}^{2}}\right\} .
\end{aligned}
$$

The total contribution of scalar particles to the NLO kernel from the diagrams in Figs. 3 and 4 is a sum of Eqs. (39) and (36)

$$
\begin{aligned}
\frac{d}{d \eta}\langle\operatorname{Tr} & \left.\left\{\hat{U}_{z_{1}}^{\eta} \hat{U}_{z_{2}}^{\dagger \eta}\right\}\right\rangle_{\text {scalars }} \\
= & -\frac{3 \alpha^{2}}{4 \pi^{4}} \int \frac{d^{2} z_{3} d^{2} z_{4}}{z_{34}^{4}}\left[-2+\frac{z_{13}^{2} z_{24}^{2}+z_{14}^{2} z_{23}^{2}}{z_{13}^{2} z_{24}^{2}-z_{14}^{2} z_{23}^{2}} \ln \frac{z_{13}^{2} z_{24}^{2}}{z_{14}^{2} z_{23}^{2}}\right] \\
& \times\left(U_{z_{3}}^{a a^{\prime}} U_{z_{4}}^{b b^{\prime}}-z_{4} \rightarrow z_{3}\right) \operatorname{Tr}\left\{\left[T^{a}, T^{b}\right] U_{z_{1}}^{\eta}\left[T^{a^{\prime}}, T^{b^{\prime}}\right] U_{z_{2}}^{\dagger \eta}\right\} \\
& -\frac{\alpha_{s}^{2} N_{c}}{4 \pi^{3}} \int d^{2} z_{3} \operatorname{Tr}\left\{T^{a} U_{z_{1}} U_{z_{3}}^{\dagger} T^{a} U_{z_{3}} U_{z_{2}}^{\dagger}\right\} \\
& \times\left\{\frac{z_{12}^{2}}{z_{13}^{2} z_{23}^{2}}\left[\ln z_{12}^{2} \mu^{2}+\frac{8}{3}\right]+\left[\frac{1}{z_{13}^{2}}-\frac{1}{z_{23}^{2}}\right] \ln \frac{z_{13}^{2}}{z_{23}^{2}}\right\} .
\end{aligned}
$$

Finally, one needs to add the contribution of diagrams without scalar-shockwave intersection shown in Fig. 5. They are proportional to the "parent dipole" $\operatorname{Tr}\left\{U_{z_{1}} U_{z_{2}}^{\dagger}\right\}$, and their contribution can be found from Eq. (40) using the requirement that the r.h.s. of the evolution equation must vanish as $z_{1} \rightarrow z_{2}$ (since $\lim _{z_{1} \rightarrow z_{2}} \hat{U}_{z_{1}}^{\eta} \hat{U}^{\eta} \dagger_{z_{2}}=1$, see the discussion below Eq. (8)). It is easy to see that the following formula for the total contribution of scalar particle fulfills this requirement: 


$$
\begin{aligned}
\frac{d}{d \eta}( & \left.\operatorname{Tr}\left\{\hat{U}_{z_{1}}^{\eta} \hat{U}_{z_{2}}^{\dagger \eta}\right\}\right)_{\text {scalars }} \\
= & -\frac{3 \alpha^{2}}{4 \pi^{4}} \int \frac{d^{2} z_{3} d^{2} z_{4}}{z_{34}^{4}}\left[-2+\frac{z_{13}^{2} z_{24}^{2}+z_{14}^{2} z_{23}^{2}}{z_{13}^{2} z_{24}^{2}-z_{14}^{2} z_{23}^{2}} \ln \frac{z_{13}^{2} z_{24}^{2}}{z_{14}^{2} z_{23}^{2}}\right] \\
& \times\left(\hat{U}_{z_{3}}^{\eta, a a^{\prime}} \hat{U}_{z_{4}}^{\eta, b b^{\prime}}-z_{4} \rightarrow z_{3}\right) \operatorname{Tr}\left\{\left[T^{a}, T^{b}\right] \hat{U}_{z_{1}}^{\eta}\left[T^{a^{\prime}}, T^{b^{\prime}}\right] \hat{U}_{z_{2}}^{\dagger \eta}\right\} \\
& -\frac{\alpha_{s}^{2} N_{c}}{4 \pi^{3}} \int d^{2} z_{3}\left[\operatorname{Tr}\left\{T^{a} \hat{U}_{z_{1}}^{\eta} \hat{U}_{z_{3}}^{\dagger \eta} T^{a} \hat{U}_{z_{3}} \hat{U}_{z_{2}}^{\dagger \eta}\right\}-N_{c} \operatorname{Tr}\left\{\hat{U}_{z_{1}}^{\eta} \hat{U}_{z_{2}}^{\dagger \eta}\right\}\right] \\
& \times\left\{\frac{z_{12}^{2}}{z_{13}^{2} z_{23}^{2}}\left[\ln z_{12}^{2} \mu^{2}+\frac{8}{3}\right]+\left[\frac{1}{z_{13}^{2}}-\frac{1}{z_{23}^{2}}\right] \ln \frac{z_{13}^{2}}{z_{23}^{2}}\right\} .
\end{aligned}
$$

Note that we have written this equation in the operator form by promoting the shock-wave fields in the r.h.s. of Eq. (40) to operators.

\subsection{Gluino contribution}

The diagrams for the gluino contribution to the NLO kernel are shown in Fig. 6. The gluino propagator in the shock-wave background has the form

$$
\begin{aligned}
\left\langle\lambda_{\alpha}^{a I}(x) \bar{\lambda}_{\dot{\alpha}}^{b J}(y)\right\rangle & \\
= & \theta\left(x_{*} y_{*}\right) \int d^{4} k e^{-i k \cdot(x-y)} \frac{i k_{\mu} \bar{\sigma}_{\alpha \dot{\alpha}}^{\mu}}{k^{2}+i \epsilon} \delta^{a b} \delta^{I J} \\
& +\delta_{I J} \theta\left(x_{*}\right) \theta\left(-y_{*}\right) \\
& \times \int_{0}^{\infty} d \alpha \frac{e^{-i \alpha(x-y)}}{2 \alpha^{2} s}\left(x_{\perp}\left|\left(\alpha \bar{p}_{1}+\bar{p}_{\perp}\right) e^{-i \frac{p_{\perp}^{2}}{\alpha s} x_{*}} p_{2} U^{a b} e^{i \frac{p_{\perp}^{2}}{\alpha s} y_{*}}\left(\alpha \bar{p}_{1}+\bar{p}_{\perp}\right)\right| y_{\perp}\right)_{\alpha \dot{\alpha}} \\
& +\delta_{I J} \theta\left(-x_{*}\right) \theta\left(y_{*}\right) \\
& \times \int_{0}^{\infty} d \alpha \frac{e^{i \alpha(x-y)}}{2 \alpha^{2} s}\left(x_{\perp} \mid\left(\alpha \bar{p}_{1}+\bar{p}_{\perp}\right) e^{i \frac{p_{\perp}^{2}}{\alpha s} x_{*}} p_{2} U^{\dagger a b} e^{\left.-i \frac{p_{\perp}^{2}}{\alpha s} y_{*}\left(\alpha \bar{p}_{1}+\bar{p}_{\perp}\right) \mid y_{\perp}\right)_{\alpha \dot{\alpha}},}\right.
\end{aligned}
$$

where $\bar{p}_{\alpha \dot{\alpha}} \equiv p_{\mu} \bar{\sigma}_{\alpha \dot{\alpha}}^{\mu}$ and $p^{\dot{\alpha} \alpha} \equiv p^{\mu} \bar{\sigma}_{\mu}^{\dot{\alpha} \alpha}$.

This propagator has the same form as the quark propagator in the shock-wave background in QCD so one can use the result for the quark part of the NLO BK kernel in QCD calculated in Refs. [17,18]. We replace $\operatorname{tr}\left\{t^{a} U_{z_{3}} t^{b} U_{z_{4}}^{\dagger}\right\}$ for quarks in the fundamental representation by $2 \operatorname{Tr}\left\{T^{a} U_{z_{3}} T^{b} U_{z_{4}}^{\dagger}\right\}$ for gluinos in the adjoint representation (and $\operatorname{tr}\left\{t^{a} U_{z_{1}} t^{b} U_{z_{2}}^{\dagger}\right\}$ by $\operatorname{Tr}\left\{T^{a} U_{z_{1}} T^{b} U_{z_{2}}^{\dagger}\right\}$ for Wilson loop as usual) and obtain

$$
\begin{aligned}
\frac{d}{d \eta} \operatorname{Tr} & \left\{\hat{U}_{z_{1}}^{\eta} \hat{U}_{z_{2}}^{\dagger \eta}\right\} \text { gluino } \\
= & \frac{2 \alpha_{s}^{2} N_{c}}{3 \pi^{3}} \int d^{2} z_{3}\left[\operatorname{Tr}\left\{T^{a} \hat{U}_{z_{1}}^{\eta} \hat{U}_{z_{3}}^{\dagger \eta} T^{a} \hat{U}_{z_{3}}^{\eta} \hat{U}_{z_{2}}^{\dagger \eta}\right\}-N_{c} \operatorname{Tr}\left\{\hat{U}_{z_{1}}^{\eta} \hat{U}_{z_{2}}^{\dagger \eta}\right\}\right] \\
& \times\left[-\frac{z_{12}^{2}}{z_{13}^{2} z_{23}^{2}}\left(\ln z_{12}^{2} \mu^{2}+\frac{5}{3}\right)+\frac{z_{13}^{2}-z_{23}^{2}}{z_{13}^{2} z_{23}^{2}} \ln \frac{z_{13}^{2}}{z_{23}^{2}}\right]
\end{aligned}
$$



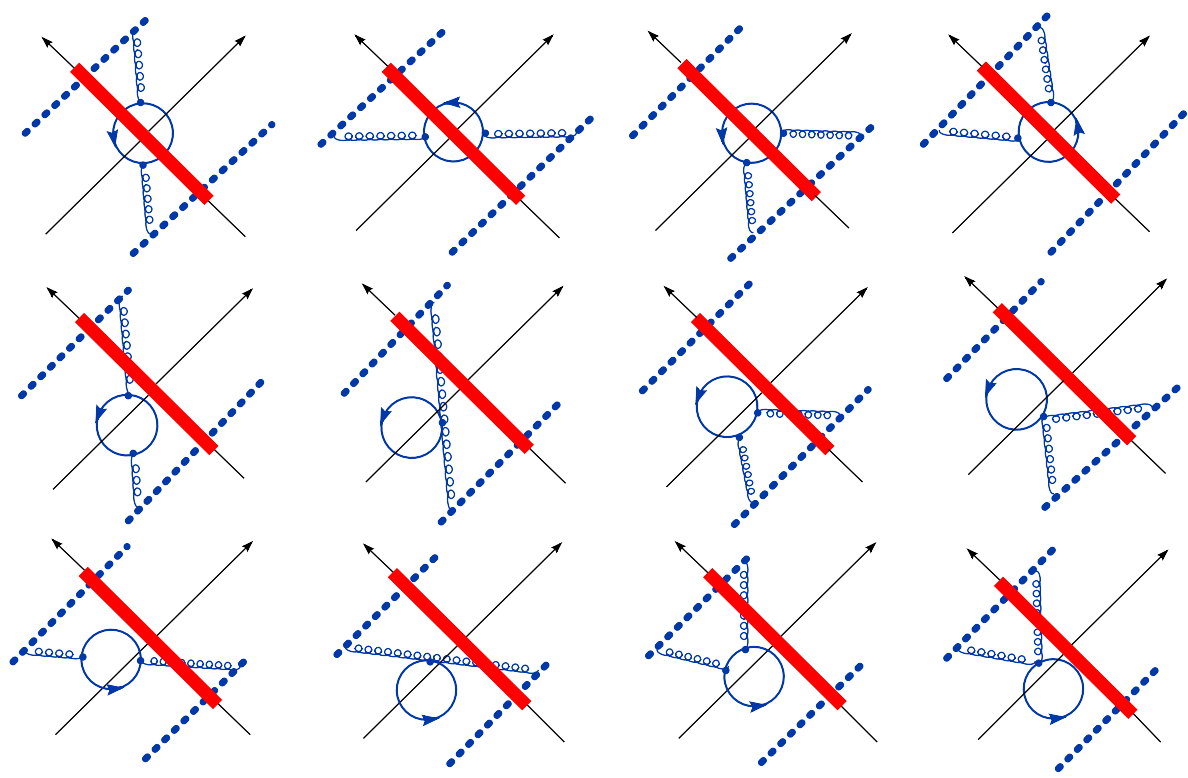

Fig. 6. Diagrams with the gluino loop.

$$
\begin{aligned}
& -\frac{2 \alpha_{s}^{2}}{\pi^{4}} \int \frac{d^{2} z_{3} d^{2} z_{4}}{z_{34}^{4}}\left\{1-\frac{z_{14}^{2} z_{23}^{2}+z_{24}^{2} z_{13}^{2}-z_{12}^{2} z_{34}^{2}}{2\left(z_{13}^{2} z_{24}^{2}-z_{14}^{2} z_{23}^{2}\right)} \ln \frac{z_{13}^{2} z_{24}^{2}}{z_{14}^{2} z_{23}^{2}}\right\}\left(\hat{U}_{z_{3}}^{\eta}\right)^{a a^{\prime}} \\
& \times\left(\hat{U}_{z_{4}}^{\eta}-\hat{U}_{z_{3}}^{\eta}\right)^{b b^{\prime}} \operatorname{Tr}\left\{\left[T^{a}, T^{b}\right] \hat{U}_{z_{1}}^{\eta}\left[T^{a^{\prime}}, T^{b^{\prime}}\right] \hat{U}_{z_{2}}^{\dagger \eta}\right\} .
\end{aligned}
$$

\subsection{The $N=4$ kernel}

Now we are in a position to assemble the NLO BK kernel in $\mathcal{N}=4 \mathrm{SYM}$. Adding the gluon contribution (21), the scalar part (41), and the gluino term (42) we obtain

$$
\begin{aligned}
\frac{d}{d \eta} \operatorname{Tr} & \left\{\hat{U}_{z_{1}}^{\eta} \hat{U}_{z_{2}}^{\dagger \eta}\right\} \\
= & \frac{\alpha_{s}}{\pi^{2}} \int d^{2} z_{3} \frac{z_{12}^{2}}{z_{13}^{2} z_{23}^{2}}\left[1-\frac{\alpha_{s} N_{c}}{4 \pi}\left(\frac{\pi^{2}}{3}+2 \ln \frac{z_{12}^{2}}{z_{13}^{2}} \ln \frac{z_{12}^{2}}{z_{23}^{2}}\right)\right] \\
& \times\left[\operatorname{Tr}\left\{T^{a} \hat{U}_{z_{1}}^{\eta} \hat{U}_{z_{3}}^{\dagger \eta} T^{a} \hat{U}_{z_{3}} \hat{U}_{z_{2}}^{\dagger \eta}\right\}-N_{c} \operatorname{Tr}\left\{\hat{U}_{z_{1}}^{\eta} \hat{U}_{z_{2}}^{\dagger \eta}\right\}\right] \\
& -\frac{\alpha_{s}^{2}}{8 \pi^{4}} \int \frac{d^{2} z_{3} d^{2} z_{4}}{z_{34}^{4}}\left(\hat{U}_{z_{3}}^{\eta}\right)^{a a^{\prime}}\left\{\left[\frac{z_{12}^{2} z_{34}^{2}}{z_{13}^{2} z_{24}^{2}}\left(1+\frac{z_{12}^{2} z_{34}^{2}}{z_{13}^{2} z_{24}^{2}-z_{23}^{2} z_{14}^{2}}\right) \ln \frac{z_{13}^{2} z_{24}^{2}}{z_{14}^{2} z_{23}^{2}}+z_{3} \leftrightarrow z_{4}\right]\right. \\
& \times \operatorname{Tr}\left\{\left[T^{a}, T^{b}\right] \hat{U}_{z_{1}}^{\eta} T^{a^{\prime}} T^{b^{\prime}} \hat{U}_{z_{2}}^{\dagger \eta}+T^{b} T^{a} \hat{U}_{z_{1}}^{\eta}\left[T^{b^{\prime}}, T^{a^{\prime}}\right] \hat{U}_{z_{2}}^{\dagger \eta}\right\}\left(\hat{U}_{z_{4}}^{\eta}-\hat{U}_{z_{3}}^{\eta}\right)^{b b^{\prime}} \\
& +\left[\frac{z_{12}^{2} z_{34}^{2}}{z_{13}^{2} z_{24}^{2}}\left(1+\frac{z_{12}^{2} z_{34}^{2}}{z_{13}^{2} z_{24}^{2}-z_{23}^{2} z_{14}^{2}}\right) \ln \frac{z_{13}^{2} z_{24}^{2}}{z_{14}^{2} z_{23}^{2}}-z_{3} \leftrightarrow z_{4}\right] \\
& \left.\times \operatorname{Tr}\left\{\left[T^{a}, T^{b}\right] \hat{U}_{z_{1}}^{\eta} T^{a^{\prime}} T^{b^{\prime}} \hat{U}_{z_{2}}^{\dagger \eta}+T^{b} T^{a} \hat{U}_{z_{1}}^{\eta}\left[T^{b^{\prime}}, T^{a^{\prime}}\right] \hat{U}_{z_{2}}^{\dagger \eta}\right\}\left(\hat{U}_{z_{4}}^{\eta}\right)^{b b^{\prime}}\right\} .
\end{aligned}
$$


Using Eq. (114) from Appendix E one can rewrite this equation as follows:

$$
\begin{aligned}
\frac{d}{d \eta} \operatorname{Tr} & \left\{\hat{U}_{z_{1}}^{\eta} \hat{U}_{z_{2}}^{\dagger \eta}\right\} \\
= & \frac{\alpha_{s}}{\pi^{2}} \int d^{2} z_{3} \frac{z_{12}^{2}}{z_{13}^{2} z_{23}^{2}}\left\{1-\frac{\alpha_{s} N_{c}}{4 \pi}\left[\frac{\pi^{2}}{3}+2 \ln \frac{z_{13}^{2}}{z_{12}^{2}} \ln \frac{z_{23}^{2}}{z_{12}^{2}}\right]\right\} \\
& \times\left[\operatorname{Tr}\left\{T^{a} \hat{U}_{z_{1}}^{\eta} \hat{U}_{z_{3}}^{\dagger \eta} T^{a} \hat{U}_{z_{3}}^{\eta} \hat{U}_{z_{2}}^{\dagger \eta}\right\}-N_{c} \operatorname{Tr}\left\{\hat{U}_{z_{1}}^{\eta} \hat{U}_{z_{2}}^{\dagger \eta}\right\}\right] \\
& -\frac{\alpha_{s}^{2}}{4 \pi^{4}} \int \frac{d^{2} z_{3} d^{2} z_{4}}{z_{34}^{4}} \frac{z_{12}^{2} z_{34}^{2}}{z_{13}^{2} z_{24}^{2}}\left[1+\frac{z_{12}^{2} z_{34}^{2}}{z_{13}^{2} z_{24}^{2}-z_{23}^{2} z_{14}^{2}}\right] \ln \frac{z_{13}^{2} z_{24}^{2}}{z_{14}^{2} z_{23}^{2}} \\
& \times \operatorname{Tr}\left\{\left[T^{a}, T^{b}\right] \hat{U}_{z_{1}}^{\eta} T^{a^{\prime}} T^{b^{\prime}} \hat{U}_{z_{2}}^{\dagger \eta}+T^{b} T^{a} \hat{U}_{z_{1}}^{\eta}\left[T^{b^{\prime}}, T^{a^{\prime}}\right] \hat{U}_{z_{2}}^{\dagger \eta}\right\}\left(\hat{U}_{z_{3}}^{\eta}\right)^{a a^{\prime}}\left(\hat{U}_{z_{4}}^{\eta}-\hat{U}_{z_{3}}^{\eta}\right)^{b b^{\prime}} .
\end{aligned}
$$

All terms in the r.h.s. of this equation are Möbius-invariant except the double-log term proportional to $\ln \frac{z_{13}^{2}}{z_{12}^{2}} \ln \frac{z_{23}^{2}}{z_{12}^{2}}$. As we discussed in the Introduction, the reason for this non-invariance is the cutoff in the longitudinal direction which violates the formal invariance of the non-cut Wilson lines.

It is worth noting that conformal and non-conformal terms come from graphs with different topology: the conformal terms come from $1 \rightarrow 3$ dipoles diagrams (see Fig. 6 in Ref. [11]) which describe the dipole creation while the non-conformal double-log term comes from the $1 \rightarrow 2$ dipole transitions (Fig. 9 in Ref. [11]) which can be regarded as a combination of dipole creation and dipole recombination.

\section{Conformal dipole and conformal NLO kernel}

A we discussed in the Introduction, it is possible to define the composite conformal dipole operator order by order in perturbation theory in such a way that the evolution equation for this operator would be Möbius-invariant. The form of this operator can be guessed from the expression (99) (see the discussion in Appendix B)

$$
\begin{aligned}
{\left[\operatorname{Tr}\left\{\hat{U}_{z_{1}}^{\eta} \hat{U}_{z_{2}}^{\dagger \eta}\right\}\right]^{\mathrm{conf}} } & \\
= & \operatorname{Tr}\left\{\hat{U}_{z_{1}}^{\eta} \hat{U}_{z_{2}}^{\dagger \eta}\right\}+\frac{\alpha_{s}}{2 \pi^{2}} \int d^{2} z_{3} \frac{z_{12}^{2}}{z_{13}^{2} z_{23}^{2}}\left[\operatorname{Tr}\left\{T^{n} \hat{U}_{z_{1}}^{\eta} \hat{U}_{z_{3}}^{\dagger \eta} T^{n} \hat{U}_{z_{3}}^{\eta} \hat{U}_{z_{2}}^{\dagger \eta}\right\}\right. \\
& \left.-N_{c} \operatorname{Tr}\left\{\hat{U}_{z_{1}}^{\eta} \hat{U}_{z_{2}}^{\dagger \eta}\right\}\right] \ln \frac{a z_{12}^{2}}{z_{13}^{2} z_{23}^{2}} .
\end{aligned}
$$

Let us find the NLO evolution kernel for this operator and demonstrate that it is conformal.

For the evolution of the composite operator (45) we get

$$
\begin{aligned}
\frac{d}{d \eta}\left[\operatorname{Tr}\left\{\hat{U}_{z_{1}}^{\eta} \hat{U}_{z_{2}}^{\dagger \eta}\right\}\right]^{\mathrm{conf}} \\
=\frac{d}{d \eta} \operatorname{Tr}\left\{\hat{U}_{z_{1}}^{\eta} \hat{U}_{z_{2}}^{\dagger \eta}\right\}+\frac{\alpha_{s}}{2 \pi^{2}} \int d^{2} z_{3} \frac{z_{12}^{2}}{z_{13}^{2} z_{23}^{2}}\left[\frac{d}{d \eta} \operatorname{Tr}\left\{T^{a} \hat{U}_{z_{1}}^{\eta} \hat{U}_{z_{3}}^{\dagger \eta} T^{a} \hat{U}_{z_{3}}^{\eta} \hat{U}_{z_{2}}^{\dagger \eta}\right\}\right. \\
\left.\quad-N_{c} \frac{d}{d \eta} \operatorname{Tr}\left\{\hat{U}_{z_{1}}^{\eta} \hat{U}_{z_{2}}^{\dagger \eta}\right\}\right] \ln \frac{a z_{12}^{2}}{z_{13}^{2} z_{23}^{2}} .
\end{aligned}
$$


Writing down the evolution of the four-Wilson-line operator (105) calculated in Appendix C we obtain

$$
\begin{aligned}
& \frac{d}{d \eta}\left[\operatorname{Tr}\left\{\hat{U}_{z_{1}}^{\eta} \hat{U}_{z_{2}}^{\dagger \eta}\right\}\right]^{\operatorname{conf}} \\
&=\frac{\alpha_{s}}{\pi^{2}} \int d^{2} z_{3} \frac{z_{12}^{2}}{z_{13}^{2} z_{23}^{2}}\left\{1+\frac{\alpha_{s} N_{c}}{4 \pi}\left[-\frac{\pi^{2}}{3}-2 \ln \frac{z_{13}^{2}}{z_{12}^{2}} \ln \frac{z_{23}^{2}}{z_{12}^{2}}\right]\right\} \\
& \times\left[\operatorname{Tr}\left\{T^{a} \hat{U}_{z_{1}}^{\eta} \hat{U}_{z_{3}}^{\dagger \eta} T^{a} \hat{U}_{z_{3}}^{\eta} \hat{U}_{z_{2}}^{\dagger \eta}\right\}-N_{c} \operatorname{Tr}\left\{\hat{U}_{z_{1}}^{\eta} \hat{U}_{z_{2}}^{\dagger \eta}\right\}\right] \\
&+\frac{\alpha_{s}^{2}}{8 \pi^{4}} \int d^{2} z_{3} d^{2} z_{4}\left(\hat{U}_{z_{3}}^{\eta}\right)^{a a^{\prime}}\left[\frac { z _ { 1 2 } ^ { 2 } } { z _ { 1 4 } ^ { 2 } z _ { 2 4 } ^ { 2 } } \operatorname { T r } \left\{T^{a} T^{b} \hat{U}_{z_{1}}^{\eta} T^{a^{\prime}} T^{b^{\prime}} \hat{U}_{z_{2}}^{\dagger \eta}\right.\right. \\
&\left.+T^{b} T^{a} \hat{U}_{z_{1}}^{\eta} T^{b^{\prime}} T^{a^{\prime}} \hat{U}_{z_{2}}^{\dagger \eta}\right\}\left(2 \hat{U}_{z_{4}}^{\eta}-\hat{U}_{z_{1}}^{\eta}-\hat{U}_{z_{2}}^{\eta}\right)^{b b^{\prime}} \\
&-\frac{z_{13}^{2}}{z_{14}^{2} z_{34}^{2}} \operatorname{Tr}\left\{T^{a} T^{b} \hat{U}_{z_{1}}^{\eta}\left[T^{a^{\prime}}, T^{b^{\prime}}\right] \hat{U}_{z_{2}}^{\dagger \eta}\right. \\
&\left.+\left[T^{b}, T^{a}\right] \hat{U}_{z_{1}}^{\eta} T^{b^{\prime}} T^{a^{\prime}} \hat{U}_{z_{2}}^{\dagger \eta}\right\}\left(2 \hat{U}_{z_{4}}^{\eta}-\hat{U}_{z_{1}}^{\eta}-\hat{U}_{z_{3}}^{\eta}\right)^{b b^{\prime}} \\
&-\frac{z_{23}^{2}}{z_{24}^{2} z_{34}^{2}} \operatorname{Tr}\left\{\left[T^{a}, T^{b}\right] \hat{U}_{z_{1}}^{\eta} T^{a^{\prime}} T^{b^{\prime}} \hat{U}_{z_{2}}^{\dagger \eta}\right. \\
&\left.\left.+T^{b} T^{a} \hat{U}_{z_{1}}^{\eta}\left[T^{b^{\prime}}, T^{a^{\prime}}\right] \hat{U}_{z_{2}}^{\dagger \eta}\right\}\left(2 \hat{U}_{z_{4}}^{\eta}-\hat{U}_{z_{2}}^{\eta}-\hat{U}_{z_{3}}^{\eta}\right)^{b b^{\prime}}\right] \frac{z_{12}^{2}}{z_{13}^{2} z_{23}^{2}} \ln \frac{a z_{12}^{2}}{z_{13}^{2} z_{23}^{2}} \\
&-\frac{\alpha_{s}^{2} N_{c}}{2 \pi^{4}} \int d^{2} z_{3} d^{2} z_{4}\left(\operatorname{Tr}\left\{T^{a} \hat{U}_{z_{1}}^{\eta} \hat{U}_{z_{4}}^{\dagger \eta} T^{a} \hat{U}_{z_{4}}^{\eta} \hat{U}_{z_{2}}^{\dagger \eta}\right\}-N_{c} \operatorname{Tr}\left\{U_{z_{1}} U_{z_{2}}^{\dagger}\right\}\right) \\
& \times \frac{z_{12}^{2}}{z_{13}^{2} z_{23}^{2}} \frac{z_{12}^{2}}{z_{14}^{2} z_{24}^{2}} \ln \frac{a z_{12}^{2}}{z_{13}^{2} z_{23}^{2}}+\cdots,
\end{aligned}
$$

where the dots stand for the last (conformal) term in the r.h.s. of Eq. (44).

Next, we need the "counterterms" converting the four-Wilson-line operator $\operatorname{Tr}\left\{T^{a} \hat{U}_{z_{1}}^{\eta} \hat{U}_{z 3}^{\dagger \eta} T^{a} \hat{U}_{z_{3}}^{\eta} \hat{U}_{z_{2}}^{\dagger \eta}\right\}$ into the conformal operator. In principle, this should be done similarly to obtaining the "conformal dipole" (7) in Appendix C: one should expand the T-product of conformal operators in the next $\left(\alpha_{s}^{2}\right)$ order in perturbation theory and rearrange the 6-Wilson-line operators in such a way that the coefficient in front of the combination $\left[\operatorname{Tr}\left\{T^{a} \hat{U}_{z_{1}}^{\eta} \hat{U}_{z_{3}}^{\dagger \eta} T^{a} \hat{U}_{z_{3}}^{\eta} \hat{U}_{z_{2}}^{\dagger \eta}\right\}\right]^{\text {conf }}$ is conformal. Since it means the calculation of the NNLO impact factor which is a formidable task, we will use another method to get the four-Wilson-line conformal operator. We will make a guess

$$
\begin{aligned}
& {\left[\operatorname{Tr}\left\{T^{a} \hat{U}_{z_{1}}^{\eta} \hat{U}_{z_{3}}^{\dagger \eta} T^{a} \hat{U}_{z_{3}}^{\eta} \hat{U}_{z_{2}}^{\dagger \eta}\right\}-N_{c} \operatorname{Tr}\left\{\hat{U}_{z_{1}}^{\eta} \hat{U}_{z_{2}}^{\dagger \eta}\right\}\right]^{\mathrm{conf}}} \\
& =\operatorname{Tr}\left\{T^{a} \hat{U}_{z_{1}}^{\eta} \hat{U}_{z_{3}}^{\dagger \eta} T^{a} \hat{U}_{z_{3}}^{\eta} \hat{U}_{z_{2}}^{\dagger \eta}\right\}-N_{c} \operatorname{Tr}\left\{\hat{U}_{z_{1}}^{\eta} \hat{U}_{z_{2}}^{\dagger \eta}\right\} \\
& \quad+\frac{\alpha_{s}}{8 \pi^{2}} \int d^{2} z_{4}\left(\hat{U}_{z_{3}}^{\eta}\right)^{a a^{\prime}}\left\{\operatorname{Tr}\left\{T^{a} T^{b} \hat{U}_{z_{1}}^{\eta} T^{a^{\prime}} T^{b^{\prime}} \hat{U}_{z_{2}}^{\dagger \eta}+T^{b} T^{a} \hat{U}_{z_{1}}^{\eta} T^{b^{\prime}} T^{a^{\prime}} \hat{U}_{z_{2}}^{\dagger \eta}\right\}\right. \\
& \quad \times\left(2 \hat{U}_{z_{4}}^{\eta}-\hat{U}_{z_{1}}^{\eta}-\hat{U}_{z_{2}}^{\eta}\right)^{b b^{\prime}} \frac{z_{12}^{2}}{z_{14}^{2} z_{24}^{2}} \ln \left(\frac{a z_{12}^{2}}{z_{14}^{2} z_{24}^{2}}\right) \\
& \quad-\operatorname{Tr}\left\{T^{a} T^{b} \hat{U}_{z_{1}}^{\eta}\left[T^{a^{\prime}}, T^{b^{\prime}}\right] \hat{U}_{z_{2}}^{\dagger \eta}+\left[T^{b}, T^{a}\right] \hat{U}_{z_{1}}^{\eta} T^{b^{\prime}} T^{a^{\prime}} \hat{U}_{z_{2}}^{\dagger \eta}\right\}
\end{aligned}
$$




$$
\begin{aligned}
& \times\left(2 \hat{U}_{z_{4}}^{\eta}-\hat{U}_{z_{1}}^{\eta}-\hat{U}_{z_{3}}^{\eta}\right)^{b b^{\prime}} \frac{z_{13}^{2}}{z_{14}^{2} z_{34}^{2}} \ln \left(\frac{a z_{13}^{2}}{z_{14}^{2} z_{34}^{2}}\right) \\
& -\operatorname{Tr}\left\{\left[T^{a}, T^{b}\right] \hat{U}_{z_{1}}^{\eta} T^{a^{\prime}} T^{b^{\prime}} \hat{U}_{z_{2}}^{\dagger \eta}+T^{b} T^{a} \hat{U}_{z_{1}}^{\eta}\left[T^{b^{\prime}}, T^{a^{\prime}}\right] \hat{U}_{z_{2}}^{\dagger \eta}\right\} \\
& \left.\times\left(2 \hat{U}_{z_{4}}^{\eta}-\hat{U}_{z_{2}}^{\eta}-\hat{U}_{z_{3}}^{\eta}\right)^{b b^{\prime}} \frac{z_{23}^{2}}{z_{24}^{2} z_{34}^{2}} \ln \left(\frac{a z_{23}^{2}}{z_{24}^{2} z_{34}^{2}}\right)\right\} \\
& -\frac{\alpha_{s} N_{c}}{2 \pi^{2}} \int d^{2} z_{4} \frac{z_{12}^{2}}{z_{14}^{2} z_{24}^{2}}\left(\operatorname{Tr}\left\{T^{a} U_{z_{1}} U_{z_{4}}^{\dagger} T^{a} U_{z_{4}} U_{z_{2}}^{\dagger}\right\}-N_{c} \operatorname{Tr}\left\{U_{z_{1}} U_{z_{2}}^{\dagger}\right\}\right) \ln \frac{a z_{12}^{2}}{z_{14}^{2} z_{24}^{2}},
\end{aligned}
$$

and check that it leads to the conformal evolution equation (6).

Rewriting the evolution equation (47) in terms of conformal operators (45) and (48) we obtain

$$
\begin{aligned}
& \frac{d}{d \eta}\left[\operatorname{Tr}\left\{\hat{U}_{z_{1}}^{\eta} \hat{U}_{z_{2}}^{\dagger \eta}\right\}\right]^{\mathrm{conf}} \\
& =\frac{\alpha_{s}}{\pi^{2}} \int d^{2} z_{3} \frac{z_{12}^{2}}{z_{13}^{2} z_{23}^{2}}\left\{1+\frac{\alpha_{s} N_{c}}{4 \pi}\left[-\frac{\pi^{2}}{3}-2 \ln \frac{z_{12}^{2}}{z_{13}^{2}} \ln \frac{z_{12}^{2}}{z_{23}^{2}}\right]\right\} \\
& \times\left[\operatorname{Tr}\left\{T^{a} \hat{U}_{z_{1}}^{\eta} \hat{U}_{z_{3}}^{\dagger \eta} T^{a} \hat{U}_{z_{3}}^{\eta} \hat{U}_{z_{2}}^{\dagger \eta}\right\}-N_{c} \operatorname{Tr}\left\{\hat{U}_{z_{1}}^{\eta} \hat{U}_{z_{2}}^{\dagger \eta}\right\}\right]^{\mathrm{conf}} \\
& +\frac{\alpha_{s}^{2}}{8 \pi^{4}} \int d^{2} z_{3} d^{2} z_{4} \frac{z_{12}^{2}}{z_{13}^{2} z_{23}^{2}}\left\{( \hat { U } _ { z _ { 3 } } ^ { \eta } ) ^ { a a ^ { \prime } } \operatorname { T r } \left\{T^{a} T^{b} \hat{U}_{z_{1}}^{\eta} T^{a^{\prime}} T^{b^{\prime}} \hat{U}_{z_{2}}^{\dagger \eta}\right.\right. \\
& \left.+T^{b} T^{a} \hat{U}_{z_{1}}^{\eta} T^{b^{\prime}} T^{a^{\prime}} \hat{U}_{z_{2}}^{\dagger \eta}\right\} \\
& \times\left(\hat{U}_{z_{1}}^{\eta}+\hat{U}_{z_{2}}^{\eta}\right)^{b b^{\prime}} \frac{z_{12}^{2}}{z_{14}^{2} z_{24}^{2}} \ln \left(\frac{z_{13}^{2} z_{23}^{2}}{z_{14}^{2} z_{24}^{2}}\right) \\
& +4\left(\hat{U}_{z_{3}}^{\eta}\right)^{a a^{\prime}}\left(\hat{U}_{z_{4}}^{\eta}-\hat{U}_{z_{3}}^{\eta}\right)^{b b^{\prime}} \operatorname{Tr}\left\{\left[T^{a}, T^{b}\right] \hat{U}_{z_{1}}^{\eta} T^{a^{\prime}} T^{b^{\prime}} \hat{U}_{z_{2}}^{\dagger \eta}+T^{b} T^{a} \hat{U}_{z_{1}}^{\eta}\left[T^{b^{\prime}}, T^{a^{\prime}}\right] \hat{U}_{z_{2}}^{\dagger \eta}\right\} \\
& \times \frac{z_{23}^{2}}{z_{24}^{2} z_{34}^{2}} \ln \left(\frac{z_{14}^{2} z_{23}^{2}}{z_{12}^{2} z_{34}^{2}}\right) \\
& +4\left(\hat{U}_{z_{3}}^{\eta}\right)^{a a^{\prime}}\left(\hat{U}_{z_{3}}^{\eta}\right)^{b b^{\prime}} \operatorname{Tr}\left\{\left[T^{a}, T^{b}\right] \hat{U}_{z_{1}}^{\eta} T^{a^{\prime}} T^{b^{\prime}} \hat{U}_{z_{2}}^{\dagger \eta}+T^{b} T^{a} \hat{U}_{z_{1}}^{\eta}\left[T^{b^{\prime}}, T^{a^{\prime}}\right] \hat{U}_{z_{2}}^{\dagger \eta}\right\} \\
& \times \frac{z_{23}^{2}}{z_{24}^{2} z_{34}^{2}} \ln \left(\frac{z_{14}^{2} z_{23}^{2}}{z_{12}^{2} z_{34}^{2}}\right) \\
& -\left(\hat{U}_{z_{1}}^{\eta}+\hat{U}_{z_{3}}^{\eta}\right)^{a a^{\prime}}\left(\hat{U}_{z_{3}}^{\eta}\right)^{b b^{\prime}} \operatorname{Tr}\left\{\left[T^{a}, T^{b}\right] \hat{U}_{z_{1}}^{\eta} T^{a^{\prime}} T^{b^{\prime}} \hat{U}_{z_{2}}^{\dagger \eta}+T^{b} T^{a} \hat{U}_{z_{1}}^{\eta}\left[T^{b^{\prime}}, T^{a^{\prime}}\right] \hat{U}_{z_{2}}^{\dagger \eta}\right\} \\
& \times \frac{z_{13}^{2}}{z_{14}^{2} z_{34}^{2}} \ln \left(\frac{z_{13}^{4} z_{23}^{2}}{z_{12}^{2} z_{14}^{2} z_{34}^{2}}\right) \\
& -\left(\hat{U}_{z_{3}}^{\eta}\right)^{a a^{\prime}}\left(\hat{U}_{z_{2}}^{\eta}+\hat{U}_{z_{3}}^{\eta}\right)^{b b^{\prime}} \operatorname{Tr}\left\{\left[T^{a}, T^{b}\right] \hat{U}_{z_{1}}^{\eta} T^{a^{\prime}} T^{b^{\prime}} \hat{U}_{z_{2}}^{\dagger \eta}+T^{b} T^{a} \hat{U}_{z_{1}}^{\eta}\left[T^{b^{\prime}}, T^{a^{\prime}}\right] \hat{U}_{z_{2}}^{\dagger \eta}\right\} \\
& \left.\times \frac{z_{23}^{2}}{z_{24}^{2} z_{34}^{2}} \ln \left(\frac{z_{23}^{4} z_{13}^{2}}{z_{12}^{2} z_{24}^{2} z_{34}^{2}}\right)\right\} \\
& +\frac{\alpha_{s}^{2} N_{c}}{2 \pi^{4}} \int d^{2} z_{3} d^{2} z_{4} \frac{z_{12}^{2}}{z_{13}^{2} z_{23}^{2}} \frac{z_{12}^{2}}{z_{14}^{2} z_{24}^{2}}\left[\operatorname{Tr}\left\{T^{a} \hat{U}_{z_{1}} \hat{U}_{z_{3}}^{\dagger} T^{a} \hat{U}_{z_{3}} \hat{U}_{z_{2}}^{\dagger}\right\}\right. \\
& \left.-N_{c} \operatorname{Tr}\left\{\hat{U}_{z_{1}} \hat{U}_{z_{2}}^{\dagger}\right\}\right] \ln \frac{z_{14}^{2} z_{24}^{2}}{z_{13}^{2} z_{23}^{2}}+\cdots \text {. }
\end{aligned}
$$


Note that with our accuracy we do not need to specify the form of "counterterms" for the conformal composite operators in the $\alpha_{s}^{2}$ term in the r.h.s. of this equation.

Using Eq. (107) and the integral (116) from Appendix E we get

$$
\begin{aligned}
\frac{d}{d \eta}[ & \left.\operatorname{Tr}\left\{\hat{U}_{z_{1}}^{\eta} \hat{U}_{z_{2}}^{\dagger \eta}\right\}\right]^{\operatorname{conf}} \\
= & \frac{\alpha_{s}}{\pi^{2}} \int d^{2} z_{3} \frac{z_{12}^{2}}{z_{13}^{2} z_{23}^{2}}\left\{1+\frac{\alpha_{s} N_{c}}{4 \pi}\left[-\frac{\pi^{2}}{3}-2 \ln \frac{z_{12}^{2}}{z_{13}^{2}} \ln \frac{z_{12}^{2}}{z_{23}^{2}}\right]\right\} \\
& \times\left[\operatorname{Tr}\left\{T^{a} \hat{U}_{z_{1}}^{\eta} \hat{U}_{z_{3}}^{\dagger \eta} T^{a} \hat{U}_{z_{3}}^{\eta} \hat{U}_{z_{2}}^{\dagger \eta}\right\}-N_{c} \operatorname{Tr}\left\{\hat{U}_{z_{1}}^{\eta} \hat{U}_{z_{2}}^{\dagger \eta}\right\}\right]^{\mathrm{conf}} \\
& +\frac{\alpha_{s}}{2 \pi^{4}} \int d^{2} z_{3} d^{2} z_{4} \frac{z_{12}^{2}}{z_{13}^{2} z_{24}^{2} z_{34}^{2}}\left(\hat{U}_{z_{3}}^{\eta}\right)^{a a^{\prime}}\left(\hat{U}_{z_{4}}^{\eta}-\hat{U}_{z_{3}}^{\eta}\right)^{b b^{\prime}} \\
& \times \operatorname{Tr}\left\{\left[T^{a}, T^{b}\right] \hat{U}_{z_{1}}^{\eta} T^{a^{\prime}} T^{b^{\prime}} \hat{U}_{z_{2}}^{\dagger \eta}+T^{b} T^{a} \hat{U}_{z_{1}}^{\eta}\left[T^{b^{\prime}}, T^{a^{\prime}}\right] \hat{U}_{z_{2}}^{\dagger \eta}\right\} \ln \frac{z_{14}^{2} z_{23}^{2}}{z_{12}^{2} z_{34}^{2}} \\
& +\frac{\alpha_{s} N_{c}}{2 \pi^{3}} \int d^{2} z_{3} \frac{z_{12}^{2}}{z_{13}^{2} z_{23}^{2}} \ln \frac{z_{12}^{2}}{z_{13}^{2}} \ln \frac{z_{12}^{2}}{z_{23}^{2}} \operatorname{Tr}\left\{T^{a} \hat{U}_{z_{1}}^{\eta} \hat{U}_{z_{3}}^{\dagger \eta} T^{a} \hat{U}_{z_{3}}^{\eta} \hat{U}_{z_{2}}^{\dagger \eta}\right\} \\
& -\frac{\alpha_{s} N_{c}^{2}}{\pi^{3}} 2 \zeta(3) \operatorname{Tr}\left\{\hat{U}_{z_{1}}^{\eta} \hat{U}_{z_{2}}^{\dagger \eta}\right\}+\cdots \\
= & \frac{\alpha_{s}}{\pi^{2}} \int d^{2} z_{3} \frac{z_{12}^{2}}{z_{13}^{2} z_{23}^{2}}\left\{1-\frac{\alpha_{s} N_{c}}{4 \pi} \frac{\pi^{2}}{3}\right\}\left[\operatorname{Tr}\left\{T^{a} \hat{U}_{z_{1}}^{\eta} \hat{U}_{z_{3}}^{\dagger \eta} T^{a} \hat{U}_{z_{3}}^{\eta} \hat{U}_{z_{2}}^{\dagger \eta}\right\}-N_{c} \operatorname{Tr}\left\{\hat{U}_{z_{1}}^{\eta} \hat{U}_{z_{2}}^{\dagger \eta}\right\}\right]^{\mathrm{conf}} \\
& +\frac{\alpha_{s}}{2 \pi^{4}} \int d^{2} z_{3} d^{2} z_{4} \frac{z_{12}^{2}}{z_{13}^{2} z_{24}^{2} z_{34}^{2}}\left(\hat{U}_{z_{3}}^{\eta}\right)^{a a^{\prime}}\left(\hat{U}_{z_{4}}^{\eta}-\hat{U}_{z_{3}}^{\eta}\right)^{b b^{\prime}} \\
& \times \operatorname{Tr}\left\{\left[T^{a}, T^{b}\right] \hat{U}_{z_{1}}^{\eta} T^{a^{\prime}} T^{b^{\prime}} \hat{U}_{z_{2}}^{\dagger \eta}+T^{b} T^{a} \hat{U}_{z_{1}}^{\eta}\left[T^{b^{\prime}}, T^{a^{\prime}}\right] \hat{U}_{z_{2}}^{\dagger \eta}\right\} \ln \frac{z_{14}^{2} z_{23}^{2}}{z_{12}^{2} z_{34}^{2}}+\cdots
\end{aligned}
$$

We see that the non-conformal term double-log term $\sim \ln \frac{z_{12}^{2}}{z_{13}^{2}} \ln \frac{z_{12}^{2}}{z_{23}^{2}}$ is canceled with the correction coming from the substitution of the dipole by the composite operator (7). This confirms our expression (7) for the conformal dipole and justifies our guess for the conformal composite 4Wilson-line operator (48).

Substituting the dots in the r.h.s. of this equation for the last (conformal) term in Eq. (44) we get the final evolution equation for the conformal composite operator cited in the Introduction (6):

$$
\begin{aligned}
& \frac{d}{d \eta}\left[\operatorname{Tr}\left\{\hat{U}_{z_{1}}^{\eta} \hat{U}_{z_{2}}^{\dagger \eta}\right\}\right]^{\mathrm{conf}} \\
&=\frac{\alpha_{s}}{\pi^{2}} \int d^{2} z_{3} \frac{z_{12}^{2}}{z_{13}^{2} z_{23}^{2}}\left[1-\frac{\alpha_{s} N_{c}}{4 \pi} \frac{\pi^{2}}{3}\right]\left[\operatorname{Tr}\left\{T^{a} \hat{U}_{z_{1}}^{\eta} \hat{U}_{z_{3}}^{\dagger \eta} T^{a} \hat{U}_{z_{3}} \hat{U}_{z_{2}}^{\dagger \eta}\right\}-N_{c} \operatorname{Tr}\left\{\hat{U}_{z_{1}}^{\eta} \hat{U}_{z_{2}}^{\dagger \eta}\right\}\right]^{\mathrm{conf}} \\
& \\
& \quad-\frac{\alpha_{s}^{2}}{4 \pi^{4}} \int d^{2} z_{3} d^{2} z_{4} \frac{z_{12}^{2}}{z_{13}^{2} z_{24}^{2} z_{34}^{2}}\left\{2 \ln \frac{z_{12}^{2} z_{34}^{2}}{z_{14}^{2} z_{23}^{2}}+\left[1+\frac{z_{12}^{2} z_{34}^{2}}{z_{13}^{2} z_{24}^{2}-z_{14}^{2} z_{23}^{2}}\right] \ln \frac{z_{13}^{2} z_{24}^{2}}{z_{14}^{2} z_{23}^{2}}\right\} \\
& \times \operatorname{Tr}\left\{\left[T^{a}, T^{b}\right] \hat{U}_{z_{1}}^{\eta} T^{a^{\prime}} T^{b^{\prime}} \hat{U}_{z_{2}}^{\dagger \eta}+T^{b} T^{a} \hat{U}_{z_{1}}^{\eta}\left[T^{b^{\prime}}, T^{a^{\prime}}\right] \hat{U}_{z_{2}}^{\dagger \eta}\right\}\left[\left(\hat{U}_{z_{3}}^{\eta}\right)^{a a^{\prime}}\left(\hat{U}_{z_{4}}^{\eta}\right)^{b b^{\prime}}\right. \\
&\left.-\left(z_{4} \rightarrow z_{3}\right)\right] .
\end{aligned}
$$


At this point we would like to discuss the gauge invariance of our evolution equation (51). The Wilson-line operator is gauge-invariant up to gauge rotations at $\pm \infty$. As it was discussed in Refs. [2,5], the evolution equation should be reformulated in terms of gauge-invariant Wilson loops. In particular, $\operatorname{Tr}\left\{\hat{U}_{z_{1}}^{\eta} \hat{U}_{z_{2}}^{\dagger \eta}\right\}$ in the 1.h.s. of this equation should be promoted to

$$
\begin{aligned}
\operatorname{Tr}\left\{\hat{U}_{z_{1}}^{\eta} \hat{U}_{z_{2}}^{\dagger \eta}\right\}= & \operatorname{Tr}\left\{\left[\infty p_{1}+z_{1},-\infty p_{1}+z_{1}\right]\left[z_{1}, z_{2}\right]_{-\infty}\right. \\
& \left.\times\left[-\infty p_{1}+z_{2}, \infty p_{1}+z_{2}\right]\left[z_{2}, z_{1}\right]_{\infty}\right\}
\end{aligned}
$$

where we use the notation $\left[z_{1}, z_{2}\right]_{ \pm \infty} \equiv\left[z_{1} \pm \infty p_{1}, z_{2} \pm \infty p_{1}\right]$ and the precise form of contours connecting these points does not matter since the fields at infinity are pure gauges. We do not have a simple way to introduce these gauge links at infinity to the r.h.s. of Eq. (51) in the adjoint representation, but it can be easily done if one rewrites the adjoint traces in terms of traces in the fundamental representation using Eq. (106) from Appendix D. For example, $\operatorname{tr}\left\{U_{z_{4}} U_{z_{1}}^{\dagger}\right\} \operatorname{tr}\left\{U_{z_{2}} U_{z_{3}}^{\dagger} U_{z_{1}} U_{z_{2}}^{\dagger} U_{z_{3}} U_{z_{4}}^{\dagger}\right\}$ should be replaced by

$$
\begin{aligned}
\operatorname{tr} & \left\{\left[p_{1}+z_{4},-\infty p_{1}+z_{4}\right]\left[z_{4}, z_{1}\right]_{-\infty}\left[-\infty p_{1}+z_{1}, \infty p_{1}+z_{1}\right]\left[z_{1}, z_{4}\right]_{\infty}\right\} \\
& \times \operatorname{tr}\left\{\left[\infty p_{1}+z_{2},-\infty p_{1}+z_{2}\right]\left[z_{2}, z_{3}\right]_{-\infty}\right\} \\
& \times\left[-\infty p_{1}+z_{3}, \infty p_{1}+z_{3}\right]\left[z_{3}, z_{1}\right]_{\infty}\left[z_{1}, z_{2}\right]_{-\infty}\left[-\infty p_{1}+z_{2}, \infty p_{1}+z_{2}\right] \\
& \times\left[z_{2}, z_{3}\right]_{\infty}\left[\infty p_{1}+z_{3},-\infty p_{1}+z_{3}\right]\left[z_{3}, z_{4}\right]_{-\infty}\left[-\infty p_{1}+z_{4}, \infty p_{1}+z_{4}\right]\left[z_{4}, z_{2}\right]_{\infty},
\end{aligned}
$$

and similarly for other traces in the r.h.s. of Eq. (107). With this replacement, the evolution equation (51) is gauge-invariant.

\section{Comparison to NLO BFKL}

In this section we compare our kernel with the forward NLO BFKL results for $\mathcal{N}=4 \mathrm{SYM}$ [1]. To compare to the BFKL amplitude of gluon-gluon scattering at high energies we need to expand our Wilson lines up to two-gluon accuracy. We define the analog of Eq. (1) in the adjoint approximation:

$$
\begin{aligned}
\hat{\mathcal{V}}^{\eta}(x, y) & =1-\frac{1}{N_{c}^{2}-1} \operatorname{Tr}\left\{\hat{U}_{x}^{\eta} \hat{U}_{y}^{\dagger \eta}\right\} \\
& =\frac{N_{c}^{2}}{N_{c}^{2}-1}[\hat{\mathcal{U}}(x, y)+\hat{\mathcal{U}}(y, x)-\hat{\mathcal{U}}(x, y) \hat{\mathcal{U}}(x, y)] \\
& \simeq \frac{N_{c}^{2}}{N_{c}^{2}-1}[\hat{\mathcal{U}}(x, y)+\hat{\mathcal{U}}(y, x)] .
\end{aligned}
$$

The corresponding conformal dipole operator in the BFKL approximation has the form

$$
\begin{aligned}
\hat{\mathcal{V}}_{\text {conf }}^{\eta}\left(z_{1}, z_{2}\right)= & \hat{\mathcal{V}}^{\eta}\left(z_{1}, z_{2}\right) \\
& +\frac{\alpha_{s} N_{c}}{4 \pi^{2}} \int d^{2} z \frac{z_{12}^{2}}{z_{13}^{2} z_{23}^{2}} \ln \frac{a z_{12}^{2}}{z_{13}^{2} z_{23}^{2}}\left[\hat{\mathcal{V}}^{\eta}\left(z_{1}, z_{3}\right)+\hat{\mathcal{V}}^{\eta}\left(z_{2}, z_{3}\right)-\hat{\mathcal{V}}^{\eta}\left(z_{1}, z_{2}\right)\right] .
\end{aligned}
$$

Using color traces (110), (111), and (109) from Appendix D it is possible to demonstrate that the conformal 4-Wilson-line operator (48) reduces to the sum of three conformal dipoles:

$$
\left[\operatorname{Tr}\left\{T^{a} \hat{U}_{z_{1}}^{\eta} \hat{U}_{z_{3}}^{\dagger \eta} T^{a} \hat{U}_{z_{3}}^{\eta} \hat{U}_{z_{2}}^{\dagger \eta}\right\}-N_{c} \operatorname{Tr}\left\{\hat{U}_{z_{1}}^{\eta} \hat{U}_{z_{2}}^{\dagger \eta}\right\}\right]^{\mathrm{conf}}
$$




$$
=-\frac{N_{c}}{2}\left(N_{c}^{2}-1\right)\left[\hat{\mathcal{V}}_{\mathrm{conf}}^{\eta}\left(z_{1}, z_{3}\right)+\hat{\mathcal{V}}_{\mathrm{conf}}^{\eta}\left(z_{2}, z_{3}\right)-\hat{\mathcal{V}}_{\mathrm{conf}}^{\eta}\left(z_{1}, z_{2}\right)\right],
$$

and therefore evolution equation (51) turns into

$$
\begin{aligned}
\frac{d}{d \eta} \hat{\mathcal{V}}_{\text {conf }}^{\eta}\left(z_{1}, z_{2}\right)= & \frac{\alpha_{s} N_{c}}{2 \pi^{2}} \int d^{2} z_{3} \frac{z_{12}^{2}}{z_{13}^{2} z_{23}^{2}}\left[1-\frac{\alpha_{s} N_{c}}{4 \pi} \frac{\pi^{2}}{3}\right] \\
& \times\left[\hat{\mathcal{V}}_{\text {conf }}^{\eta}\left(z_{1}, z_{3}\right)+\hat{\mathcal{V}}_{\text {conf }}^{\eta}\left(z_{2}, z_{3}\right)-\hat{\mathcal{V}}_{\text {conf }}^{\eta}\left(z_{1}, z_{2}\right)\right] \\
& +\frac{\alpha_{s}^{2} N_{c}^{2}}{8 \pi^{4}} \int \frac{d^{2} z_{3} d^{2} z_{4}}{z_{34}^{4}} \frac{z_{12}^{2} z_{34}^{2}}{z_{13}^{2} z_{24}^{2}} \\
& \times\left[\hat{\mathcal{V}}_{\text {conf }}^{\eta}\left(z_{3}, z_{4}\right)+\hat{\mathcal{V}}_{\text {conf }}^{\eta}\left(z_{2}, z_{4}\right)-\hat{\mathcal{V}}_{\text {conf }}^{\eta}\left(z_{2}, z_{3}\right)\right] \\
& \times\left\{2 \ln \frac{z_{12}^{2} z_{34}^{2}}{z_{14}^{2} z_{23}^{2}}+\left[1+\frac{z_{12}^{2} z_{34}^{2}}{z_{13}^{2} z_{24}^{2}-z_{14}^{2} z_{23}^{2}}\right] \ln \frac{z_{13}^{2} z_{24}^{2}}{z_{14}^{2} z_{23}^{2}}\right\} .
\end{aligned}
$$

It is convenient to change $z_{4} \leftrightarrow z_{3}$ in the second term in square brackets and to perform the integral over $z_{4}$ for the second and the third terms. One obtains:

$$
\begin{aligned}
\int \frac{d^{2} z_{3} d^{2} z_{4}}{z_{34}^{4}} \frac{z_{12}^{2} z_{34}^{2}}{z_{13}^{2} z_{24}^{2}}\left[\hat{\mathcal{V}}_{\mathrm{conf}}^{\eta}\left(z_{2}, z_{4}\right)-\hat{\mathcal{V}}_{\mathrm{conf}}^{\eta}\left(z_{2}, z_{3}\right)\right] \\
\quad \times\left\{2 \ln \frac{z_{12}^{2} z_{34}^{2}}{z_{14}^{2} z_{23}^{2}}+\left[1+\frac{z_{12}^{2} z_{34}^{2}}{z_{13}^{2} z_{24}^{2}-z_{14}^{2} z_{23}^{2}}\right] \ln \frac{z_{13}^{2} z_{24}^{2}}{z_{14}^{2} z_{23}^{2}}\right\} \\
=\int d^{2} z_{3} \hat{\mathcal{V}}_{\mathrm{coff}}^{\eta}\left(z_{2}, z_{3}\right) \frac{z_{12}^{2}}{z_{13}^{2} z_{23}^{2}} \int d^{2} z_{4}\left\{2\left[\frac{z_{13}^{2}}{z_{14}^{2} z_{34}^{2}} \ln \frac{z_{12}^{2} z_{34}^{2}}{z_{13}^{2} z_{24}^{2}}-\frac{z_{23}^{2}}{z_{24}^{2} z_{34}^{2}} \ln \frac{z_{12}^{2} z_{34}^{2}}{z_{14}^{2} z_{23}^{2}}\right]\right. \\
\left.\quad-\left[\frac{z_{13}^{2}}{z_{14}^{2} z_{34}^{2}}+\frac{z_{23}^{2}}{z_{24}^{2} z_{34}^{2}}-\frac{z_{12}^{2}}{z_{14}^{2} z_{24}^{2}}\right] \ln \frac{z_{13}^{2} z_{24}^{2}}{z_{14}^{2} z_{23}^{2}}\right\}=12 \pi \zeta(3) \hat{\mathcal{V}}_{\mathrm{conf}}^{\eta}\left(z_{1}, z_{2}\right)
\end{aligned}
$$

because

$$
\begin{aligned}
& \frac{z_{12}^{2}}{z_{13}^{2} z_{23}^{2}} \int d^{2} z_{4}\left\{2\left[\frac{z_{13}^{2}}{z_{14}^{2} z_{34}^{2}} \ln \frac{z_{12}^{2} z_{34}^{2}}{z_{13}^{2} z_{24}^{2}}-\frac{z_{23}^{2}}{z_{24}^{2} z_{34}^{2}} \ln \frac{z_{12}^{2} z_{34}^{2}}{z_{14}^{2} z_{23}^{2}}\right]\right. \\
& \left.-\left[\frac{z_{13}^{2}}{z_{14}^{2} z_{34}^{2}}+\frac{z_{23}^{2}}{z_{24}^{2} z_{34}^{2}}-\frac{z_{12}^{2}}{z_{14}^{2} z_{24}^{2}}\right] \ln \frac{z_{13}^{2} z_{24}^{2}}{z_{14}^{2} z_{23}^{2}}\right\}=12 \pi \zeta(3)\left[\delta\left(z_{13}-\delta\left(z_{23}\right)\right],\right.
\end{aligned}
$$

as it is easily seen from Eqs. (114) and (125) from Appendix E.

Using Eq. (57) one can rewrite the linearized equation (56) in the following way:

$$
\begin{aligned}
\frac{d}{d \eta} \hat{\mathcal{V}}_{\text {conf }}^{\eta}\left(z_{1}, z_{2}\right) & \\
= & \frac{\alpha_{s} N_{c}}{2 \pi^{2}} \int d^{2} z_{3} \frac{z_{12}^{2}}{z_{13}^{2} z_{23}^{2}}\left[1-\frac{\alpha_{s} N_{c}}{4 \pi} \frac{\pi^{2}}{3}\right]\left[\hat{\mathcal{V}}_{\text {conf }}^{\eta}\left(z_{1}, z_{3}\right)+\hat{\mathcal{V}}_{\text {conf }}^{\eta}\left(z_{2}, z_{3}\right)-\hat{\mathcal{V}}_{\text {conf }}^{\eta}\left(z_{1}, z_{2}\right)\right] \\
& +\frac{\alpha_{s}^{2} N_{c}^{2}}{8 \pi^{4}} \int \frac{d^{2} z_{3} d^{2} z_{4}}{z_{34}^{4}} \frac{z_{12}^{2} z_{34}^{2}}{z_{13}^{2} z_{24}^{2}}\left\{2 \ln \frac{z_{12}^{2} z_{34}^{2}}{z_{14}^{2} z_{23}^{2}}\right. \\
& \left.+\left[1+\frac{z_{12}^{2} z_{34}^{2}}{z_{13}^{2} z_{24}^{2}-z_{14}^{2} z_{23}^{2}}\right] \ln \frac{z_{13}^{2} z_{24}^{2}}{z_{14}^{2} z_{23}^{2}}\right\} \hat{\mathcal{V}}_{\text {conf }}^{\eta}\left(z_{3}, z_{4}\right)+\frac{3 \alpha_{s}^{2} N_{c}^{2}}{2 \pi^{3}} \zeta(3) \hat{\mathcal{V}}_{\text {conf }}^{\eta}\left(z_{1}, z_{2}\right)
\end{aligned}
$$


In this form the evolution equation (58) coincides with the conformal NLO BFKL equation restored in Ref. [19] from the eigenvalues calculated in Ref. [1].

\section{Evolution equation in the fundamental representation}

\subsection{In $\mathcal{N}=4 S Y M$}

For comparison with QCD let us calculate the evolution equation for color dipoles in the fundamental representation. The gluon part can be taken from Ref. [11]

$$
\begin{aligned}
\left(\frac{d}{d \eta} \operatorname{tr}\left\{\hat{U}_{z_{1}}^{\eta} \hat{U}_{z_{2}}^{\dagger \eta}\right\}\right)_{\text {gluon }} & \frac{\alpha_{s}}{2 \pi^{2}} \int d^{2} z_{3} \frac{z_{12}^{2}}{z_{13}^{2} z_{23}^{2}}\left\{1+\frac{\alpha_{s} N_{c}}{4 \pi}\left[\frac{11}{3} \ln z_{12}^{2} \mu^{2}-\frac{11}{3} \frac{z_{13}^{2}-z_{23}^{2}}{z_{12}^{2}} \ln \frac{z_{13}^{2}}{z_{23}^{2}}+\frac{64}{9}-\frac{\pi^{2}}{3}\right.\right. \\
& \left.\left.-2 \ln \frac{z_{13}^{2}}{z_{12}^{2}} \ln \frac{z_{23}^{2}}{z_{12}^{2}}\right]\right\}\left[\operatorname{tr}\left\{\hat{U}_{z_{1}}^{\eta} \hat{U}_{z_{3}}^{\dagger \eta}\right\} \operatorname{tr}\left\{\hat{U}_{z_{3}} \hat{U}_{z_{2}}^{\dagger \eta}\right\}-N_{c} \operatorname{tr}\left\{\hat{U}_{z_{1}}^{\eta} \hat{U}_{z_{2}}^{\dagger \eta}\right\}\right] \\
& +\frac{\alpha_{s}^{2}}{16 \pi^{4}} \int d^{2} z_{3} d^{2} z_{4}\left[\left\{-\frac{4}{z_{34}^{4}}+2 \frac{z_{13}^{2} z_{24}^{2}+z_{14}^{2} z_{23}^{2}-4 z_{12}^{2} z_{34}^{2}}{z_{34}^{4}\left[z_{13}^{2} z_{24}^{2}-z_{14}^{2} z_{23}^{2}\right]} \ln \frac{z_{13}^{2} z_{24}^{2}}{z_{14}^{2} z_{23}^{2}}\right.\right. \\
& \left.+\left[\left(1+\frac{z_{12}^{2} z_{34}^{2}}{z_{13}^{2} z_{24}^{2}-z_{14}^{2} z_{23}^{2}}\right) \frac{z_{12}^{2}}{z_{13}^{2} z_{24}^{2} z_{34}^{2}} \ln \frac{z_{13}^{2} z_{24}^{2}}{z_{14}^{2} z_{23}^{2}}+z_{3} \leftrightarrow z_{4}\right]\right\} \\
& \times\left[\operatorname{tr}\left\{\hat{U}_{z_{1}}^{\eta} \hat{U}_{z_{3}}^{\dagger \eta}\right\} \operatorname{tr}\left\{\hat{U}_{z_{3}} \hat{U}_{z 4}^{\dagger \eta}\right\} \operatorname{tr}\left\{\hat{U}_{z_{4}} \hat{U}_{z_{2}}^{\dagger \eta}\right\}-\operatorname{tr}\left\{\hat{U}_{z_{1}}^{\eta} \hat{U}_{z_{3}}^{\dagger \eta} \hat{U}_{z_{4}} U_{z_{2}}^{\dagger} \hat{U}_{z_{3}} \hat{U}_{z_{4}}^{\dagger \eta}\right\}-\left(z_{4} \rightarrow z_{3}\right)\right] \\
& +\left\{\frac{z_{12}^{2}}{z_{34}^{2}}\left[\frac{1}{z_{13}^{2} z_{24}^{2}}+\frac{1}{z_{23}^{2} z_{14}^{2}}\right]-\frac{z_{12}^{4}}{z_{13}^{2} z_{24}^{2} z_{14}^{2} z_{23}^{2}}\right\} \ln \frac{z_{13}^{2} z_{24}^{2}}{z_{14}^{2} z_{23}^{2}} \operatorname{tr}\left\{\hat{U}_{z_{1}}^{\eta} \hat{U}_{z_{3}}^{\dagger \eta}\right\} \\
& \left.\times \operatorname{tr}\left\{\hat{U}_{z_{3}} \hat{U}_{z_{4}}^{\dagger \eta}\right\} \operatorname{tr}\left\{\hat{U}_{z_{4}} \hat{U}_{z_{2}}^{\dagger \eta}\right\}\right] .
\end{aligned}
$$

The scalar part can be obtained from Eq. (41) by the replacement $T^{a} \rightarrow t^{a}$ and $\operatorname{Tr} \rightarrow \operatorname{tr}:$

$$
\begin{aligned}
\left(\frac{d}{d \eta}\right. & \left.\operatorname{tr}\left\{\hat{U}_{z_{1}}^{\eta} \hat{U}_{z_{2}}^{\dagger \eta}\right\}\right)_{\text {scalars }} \\
= & \frac{6 \alpha^{2}}{16 \pi^{4}} \int \frac{d^{2} z_{3} d^{2} z_{4}}{z_{34}^{4}}\left[-2+\frac{z_{13}^{2} z_{24}^{2}+z_{14}^{2} z_{23}^{2}}{z_{13}^{2} z_{24}^{2}-z_{14}^{2} z_{23}^{2}} \ln \frac{z_{13}^{2} z_{24}^{2}}{z_{14}^{2} z_{23}^{2}}\right] \\
& \times\left[\operatorname{tr}\left\{\hat{U}_{z_{1}}^{\eta} \hat{U}_{z 3}^{\dagger \eta}\right\} \operatorname{tr}\left\{\hat{U}_{z_{3}}^{\eta} \hat{U}_{z_{4}}^{\dagger \eta}\right\} \operatorname{tr}\left\{\hat{U}_{z_{4}}^{\eta} \hat{U}_{z_{2}}^{\dagger \eta}\right\}-\operatorname{tr}\left\{\hat{U}_{z_{1}}^{\eta} \hat{U}_{z_{3}}^{\dagger \eta} \hat{U}_{z_{4}}^{\eta} \hat{U}_{z_{2}}^{\dagger \eta} \hat{U}_{z_{3}}^{\eta} \hat{U}_{z_{4}}^{\dagger \eta}\right\}-\left(z_{4} \rightarrow z_{3}\right)\right] \\
& -\frac{\alpha_{s}^{2} N_{c}}{8 \pi^{3}} \int d^{2} z_{3}\left[\operatorname{tr}\left\{\hat{U}_{z_{1}}^{\eta} \hat{U}_{z 3}^{\dagger \eta}\right\} \operatorname{tr}\left\{\hat{U}_{z_{3}} \hat{U}_{z_{2}}^{\dagger \eta}\right\}-\frac{1}{N_{c}} \operatorname{tr}\left\{\hat{U}_{z_{1}}^{\eta} \hat{U}_{z_{2}}^{\dagger \eta}\right\}\right] \\
& \times\left\{\frac{z_{12}^{2}}{z_{13}^{2} z_{23}^{2}}\left[\ln z_{12}^{2} \mu^{2}+\frac{8}{3}\right]+\left[\frac{1}{z_{13}^{2}}-\frac{1}{z_{23}^{2}}\right] \ln \frac{z_{13}^{2}}{z_{23}^{2}}\right\} .
\end{aligned}
$$

Similarly, for gluino contribution one gets from Eq. (42) 


$$
\begin{aligned}
\left(\frac{d}{d \eta} \operatorname{tr}\left\{\hat{U}_{z_{1}}^{\eta} \hat{U}_{z_{2}}^{\dagger \eta}\right\}\right)_{\text {gluino }} \\
=\frac{\alpha_{s}^{2} N_{c}}{3 \pi^{3}} \int d^{2} z_{3}\left[\operatorname{tr}\left\{\hat{U}_{z_{1}}^{\eta} \hat{U}_{z_{3}}^{\dagger \eta}\right\} \operatorname{tr}\left\{\hat{U}_{z_{3}}^{\eta} \hat{U}_{z_{2}}^{\dagger \eta}\right\}-\frac{1}{N_{c}} \operatorname{tr}\left\{\hat{U}_{z_{1}}^{\eta} \hat{U}_{z_{2}}^{\dagger \eta}\right\}\right] \\
\quad \times\left[-\frac{z_{12}^{2}}{z_{13}^{2} z_{23}^{2}}\left[\ln z_{12}^{2} \mu^{2}+\frac{5}{3}\right]+\frac{z_{13}^{2}-z_{23}^{2}}{z_{13}^{2} z_{23}^{2}} \ln \frac{z_{13}^{2}}{z_{23}^{2}}\right] \\
+\frac{\alpha_{s}^{2}}{\pi^{4}} \int d^{2} z_{3} d^{2} z_{4} \frac{1}{z_{34}^{4}}\left\{1+\frac{z_{14}^{2} z_{23}^{2}+z_{24}^{2} z_{13}^{2}-z_{12}^{2} z_{34}^{2}}{2\left(z_{14}^{2} z_{23}^{2}-z_{24}^{2} z_{13}^{2}\right)} \ln \frac{z_{24}^{2} z_{13}^{2}}{z_{14}^{2} z_{23}^{2}}\right\} \\
\quad \times\left[\operatorname{tr}\left\{\hat{U}_{z_{1}}^{\eta} \hat{U}_{z_{3}}^{\dagger \eta}\right\} \operatorname{tr}\left\{\hat{U}_{z_{3}}^{\eta} \hat{U}_{z_{4}}^{\dagger \eta}\right\} \operatorname{tr}\left\{\hat{U}_{z_{4}}^{\eta} \hat{U}_{z_{2}}^{\dagger \eta}\right\}-\operatorname{tr}\left\{\hat{U}_{z_{1}}^{\eta} \hat{U}_{z_{3}}^{\dagger \eta} \hat{U}_{z_{4}}^{\eta} \hat{U}_{z_{2}}^{\dagger \eta} \hat{U}_{z_{3}}^{\eta} \hat{U}_{z_{4}}^{\dagger \eta}\right\}\right. \\
\left.\quad-\left(z_{4} \rightarrow z_{3}\right)\right] .
\end{aligned}
$$

Adding together Eqs. (59), (60) and (61) we obtain the evolution equation for the color dipole with longitudinal cutoff (8) in the fundamental representation:

$$
\begin{aligned}
\frac{d}{d \eta} \operatorname{tr} & \left\{\hat{U}_{z_{1}}^{\eta} \hat{U}_{z_{2}}^{\dagger \eta}\right\} \\
= & \frac{\alpha_{s}}{2 \pi^{2}} \int d^{2} z_{3} \frac{z_{12}^{2}}{z_{13}^{2} z_{23}^{2}}\left\{1+\frac{\alpha_{s} N_{c}}{4 \pi}\left[-\frac{\pi^{2}}{3}-2 \ln \frac{z_{13}^{2}}{z_{12}^{2}} \ln \frac{z_{23}^{2}}{z_{12}^{2}}\right]\right\} \\
& \times\left[\operatorname{tr}\left\{\hat{U}_{z_{1}}^{\eta} \hat{U}_{z_{3}}^{\dagger \eta}\right\} \operatorname{tr}\left\{\hat{U}_{z_{3}} \hat{U}_{z_{2}}^{\dagger \eta}\right\}-N_{c} \operatorname{tr}\left\{\hat{U}_{z_{1}}^{\eta} \hat{U}_{z_{2}}^{\dagger \eta}\right\}\right] \\
& +\frac{\alpha_{s}^{2}}{16 \pi^{4}} \int d^{2} z_{3} d^{2} z_{4}\left[\left\{\frac{z_{12}^{2}}{z_{13}^{2} z_{24}^{2} z_{34}^{2}}\left(1+\frac{z_{12}^{2} z_{34}^{2}}{z_{13}^{2} z_{24}^{2}-z_{14}^{2} z_{23}^{2}}\right) \ln \frac{z_{13}^{2} z_{24}^{2}}{z_{14}^{2} z_{23}^{2}}+z_{3} \leftrightarrow z_{4}\right\}\right. \\
& \times\left[\operatorname{tr}\left\{\hat{U}_{z_{1}}^{\eta} \hat{U}_{z_{3}}^{\dagger \eta}\right\} \operatorname{tr}\left\{\hat{U}_{z_{3}} \hat{U}_{z_{4}}^{\dagger \eta}\right\} \operatorname{tr}\left\{\hat{U}_{z_{4}} \hat{U}_{z_{2}}^{\dagger \eta}\right\}-\operatorname{tr}\left\{\hat{U}_{z_{1}}^{\eta} \hat{U}_{z_{3}}^{\dagger \eta} \hat{U}_{z_{4}} U_{z_{2}}^{\dagger} \hat{U}_{z_{3}} \hat{U}_{z_{4}}^{\dagger \eta}\right\}-\left(z_{4} \rightarrow z_{3}\right)\right] \\
& +\left\{\frac{z_{12}^{2}}{z_{34}^{2}}\left[\frac{1}{z_{13}^{2} z_{24}^{2}}+\frac{1}{z_{23}^{2} z_{14}^{2}}\right]-\frac{z_{12}^{4}}{z_{13}^{2} z_{24}^{2} z_{14}^{2} z_{23}^{2}}\right\} \ln \frac{z_{13}^{2} z_{24}^{2}}{z_{14}^{2} z_{23}^{2}} \operatorname{tr}\left\{\hat{U}_{z_{1}}^{\eta} \hat{U}_{z_{3}}^{\dagger \eta}\right\} \\
& \left.\times \operatorname{tr}\left\{\hat{U}_{z_{3}} \hat{U}_{z_{4}}^{\dagger \eta}\right\} \operatorname{tr}\left\{\hat{U}_{z_{4}} \hat{U}_{z_{2}}^{\dagger \eta}\right\}\right],
\end{aligned}
$$

which can be rewritten as

$$
\begin{aligned}
\frac{d}{d \eta} \operatorname{tr} & \left\{\hat{U}_{z_{1}}^{\eta} \hat{U}_{z_{2}}^{\dagger \eta}\right\} \\
= & \frac{\alpha_{s}}{2 \pi^{2}} \int d^{2} z_{3} \frac{z_{12}^{2}}{z_{13}^{2} z_{23}^{2}}\left\{1-\frac{\alpha_{s} N_{c}}{4 \pi}\left[\frac{\pi^{2}}{3}+2 \ln \frac{z_{13}^{2}}{z_{12}^{2}} \ln \frac{z_{23}^{2}}{z_{12}^{2}}\right]\right\} \\
& \times\left[\operatorname{tr}\left\{\hat{U}_{z_{1}}^{\eta} \hat{U}_{z_{3}}^{\dagger \eta}\right\} \operatorname{tr}\left\{\hat{U}_{z_{3}} \hat{U}_{z_{2}}^{\dagger \eta}\right\}-N_{c} \operatorname{tr}\left\{\hat{U}_{z_{1}}^{\eta} \hat{U}_{z_{2}}^{\dagger \eta}\right\}\right] \\
& +\frac{\alpha_{s}^{2}}{16 \pi^{4}} \int d^{2} z_{3} d^{2} z_{4} \frac{z_{12}^{2}}{z_{13}^{2} z_{24}^{2} z_{34}^{2}}\left(1+\frac{z_{12}^{2} z_{34}^{2}}{z_{13}^{2} z_{24}^{2}-z_{14}^{2} z_{23}^{2}}\right) \ln \frac{z_{13}^{2} z_{24}^{2}}{z_{14}^{2} z_{23}^{2}} \\
& \times\left[2 \operatorname{tr}\left\{\hat{U}_{z_{1}}^{\eta} \hat{U}_{z_{3}}^{\dagger \eta}\right\} \operatorname{tr}\left\{\hat{U}_{z_{3}} \hat{U}_{z_{4}}^{\dagger \eta}\right\} \operatorname{tr}\left\{\hat{U}_{z_{4}} \hat{U}_{z_{2}}^{\dagger \eta}\right\}-\operatorname{tr}\left\{\hat{U}_{z_{1}}^{\eta} \hat{U}_{z_{3}}^{\dagger \eta} \hat{U}_{z_{4}} U_{z_{2}}^{\dagger} \hat{U}_{z_{3}} \hat{U}_{z_{4}}^{\dagger \eta}\right\}\right. \\
& \left.-\operatorname{tr}\left\{\hat{U}_{z_{1}}^{\eta} \hat{U}_{z_{4}}^{\dagger \eta} \hat{U}_{z_{3}} U_{z_{2}}^{\dagger} \hat{U}_{z_{4}} \hat{U}_{z_{3}}^{\dagger \eta}\right\}-\left(z_{4} \rightarrow z_{3}\right)\right],
\end{aligned}
$$

due to Eq. (114) from Appendix D. 
The composite conformal dipole in the fundamental representation can be obtained from Eq. (7) by the usual substitution $T^{a} \rightarrow t^{a}$ and $\mathrm{Tr} \rightarrow \mathrm{tr}:$

$$
\begin{aligned}
{\left[\operatorname{tr}\left\{\hat{U}_{z_{1}}^{\eta} \hat{U}_{z_{2}}^{\dagger \eta}\right\}\right]^{\mathrm{conf}}=} & \operatorname{tr}\left\{\hat{U}_{z_{1}}^{\eta} \hat{U}_{z_{2}}^{\dagger \eta}\right\} \\
& -\frac{\alpha_{s}}{4 \pi^{2}} \int d^{2} z_{3} \frac{z_{12}^{2}}{z_{13}^{2} z_{23}^{2}} \ln \frac{a z_{12}^{2}}{z_{13}^{2} z_{23}^{2}}\left[\operatorname{tr}\left\{\hat{U}_{z_{1}}^{\eta} \hat{U}_{z_{3}}^{\dagger \eta}\right\} \operatorname{tr}\left\{\hat{U}_{z_{3}} \hat{U}_{z_{2}}^{\dagger \eta}\right\}\right. \\
& \left.-N_{c} \operatorname{tr}\left\{\hat{U}_{z_{1}}^{\eta} \hat{U}_{z_{2}}^{\dagger \eta}\right\}\right] .
\end{aligned}
$$

Similarly, the conformal 4-Wilson-line operator (48) turns to

$$
\begin{aligned}
{\left[\operatorname{tr}\left\{\hat{U}_{z_{1}}^{\eta} \hat{U}_{z_{3}}^{\dagger \eta}\right\} \operatorname{tr}\left\{U_{z_{3}} \hat{U}_{z_{2}}^{\dagger \eta}\right\}-N_{c} \operatorname{tr}\left\{\hat{U}_{z_{1}}^{\eta} \hat{U}_{z_{2}}^{\dagger \eta}\right\}\right]^{\operatorname{conf}} } \\
=\left[\operatorname{tr}\left\{\hat{U}_{z_{1}}^{\eta} \hat{U}_{z_{3}}^{\dagger \eta}\right\} \operatorname{tr}\left\{\hat{U}_{z_{3}}^{\eta} \hat{U}_{z_{2}}^{\dagger \eta}\right\}-N_{c} \operatorname{tr}\left\{\hat{U}_{z_{1}}^{\eta} \hat{U}_{z_{2}}^{\dagger \eta}\right\}\right] \\
\quad+\frac{\alpha_{s}^{2}}{4 \pi^{2}} \int d^{2} z_{4}\left\{\frac { z _ { 1 3 } ^ { 2 } } { z _ { 1 4 } ^ { 2 } z _ { 3 4 } ^ { 2 } } \left[\operatorname{tr}\left\{\hat{U}_{z_{1}}^{\eta} \hat{U}_{z_{4}}^{\dagger \eta}\right\} \operatorname{tr}\left\{\hat{U}_{z_{4}}^{\eta} \hat{U}_{z_{3}}^{\dagger \eta}\right\}\right.\right. \\
\left.\quad-N_{c} \operatorname{tr}\left\{\hat{U}_{z_{1}}^{\eta} \hat{U}_{z_{3}}^{\dagger \eta}\right\}\right] \operatorname{tr}\left\{\hat{U}_{z_{3}}^{\eta} \hat{U}_{z_{2}}^{\dagger \eta}\right\} \ln \frac{a z_{13}^{2}}{z_{14}^{2} z_{34}^{2}} \\
+\frac{z_{23}^{2}}{z_{34}^{2} z_{24}^{2}} \operatorname{tr}\left\{\hat{U}_{z_{1}}^{\eta} \hat{U}_{z_{3}}^{\dagger \eta}\right\}\left[\operatorname{tr}\left\{\hat{U}_{z_{3}}^{\eta} \hat{U}_{z_{4}}^{\dagger \eta}\right\} \operatorname{tr}\left\{\hat{U}_{z_{4}}^{\eta} \hat{U}_{z_{2}}^{\dagger \eta}\right\}-N_{c} \operatorname{tr}\left\{\hat{U}_{z_{3}}^{\eta} \hat{U}_{z_{2}}^{\dagger \eta}\right\}\right] \ln \frac{a z_{23}^{2}}{z_{24}^{2} z_{34}^{2}} \\
\quad-\frac{z_{12}^{2} N_{c}}{z_{14}^{2} z_{24}^{2}}\left[\operatorname{tr}\left\{\hat{U}_{z_{1}}^{\eta} \hat{U}_{z_{4}}^{\dagger \eta}\right\} \operatorname{tr}\left\{\hat{U}_{z_{4}}^{\eta} \hat{U}_{z_{2}}^{\dagger \eta}\right\}-N_{c} \operatorname{tr}\left\{\hat{U}_{z_{1}}^{\eta} \hat{U}_{z_{2}}^{\dagger \eta}\right\}\right] \ln \frac{a z_{12}^{2}}{z_{14}^{2} z_{24}^{2}} \\
+\frac{1}{2}\left[\frac{z_{13}^{2}}{z_{14}^{2} z_{34}^{2}} \ln \frac{a z_{13}^{2}}{z_{14}^{2} z_{34}^{2}}+\frac{z_{23}^{2}}{z_{24}^{2} z_{34}^{2}} \ln \frac{a z_{23}^{2}}{z_{34}^{2} z_{24}^{2}}-\frac{z_{12}^{2}}{z_{14}^{2} z_{24}^{2}} \ln \frac{a z_{12}^{2}}{z_{14}^{2} z_{24}^{2}}\right] \\
\left.\quad \times\left[\operatorname{tr}\left\{\hat{U}_{z_{1}}^{\eta} \hat{U}_{z_{3}}^{\dagger \eta} \hat{U}_{z_{4}}^{\eta} \hat{U}_{z_{2}}^{\dagger \eta} \hat{U}_{z_{3}}^{\eta} \hat{U}_{z_{4}}^{\dagger \eta}\right\}+\left(z_{3} \leftrightarrow z_{4}\right)-2 \operatorname{tr}\left\{\hat{U}_{z_{1}}^{\eta} \hat{U}_{z_{2}}^{\dagger \eta}\right\}\right]\right\} .
\end{aligned}
$$

Repeating the steps which lead us to Eq. (51) in Section 4 we obtain the conformal evolution equation in the fundamental representation

$$
\begin{aligned}
& \frac{d}{d \eta}\left[\operatorname{tr}\left\{\hat{U}_{z_{1}}^{\eta} \hat{U}_{z_{2}}^{\dagger \eta}\right\}\right]^{\operatorname{conf}} \\
&=\frac{\alpha_{s}}{2 \pi^{2}} \int d^{2} z_{3} \frac{z_{12}^{2}}{z_{13}^{2} z_{23}^{2}}\left[1-\frac{\alpha_{s} N_{c}}{4 \pi} \frac{\pi^{2}}{3}\right]\left[\operatorname{tr}\left\{\hat{U}_{z_{1}}^{\eta} \hat{U}_{z_{3}}^{\dagger \eta}\right\} \operatorname{tr}\left\{\hat{U}_{z_{3}}^{\eta} \hat{U}_{z_{2}}^{\dagger \eta}\right\}-N_{c} \operatorname{tr}\left\{\hat{U}_{z_{1}}^{\eta} \hat{U}_{z_{2}}^{\dagger \eta}\right\}\right]^{\operatorname{conf}} \\
&+\frac{\alpha_{s}^{2}}{16 \pi^{4}} \int d^{2} z_{3} d^{2} z_{4} \frac{z_{12}^{2}}{z_{13}^{2} z_{34}^{2} z_{24}^{2}}\left\{2 \ln \frac{z_{12}^{2} z_{34}^{2}}{z_{14}^{2} z_{23}^{2}}+\left[1+\frac{z_{12}^{2} z_{34}^{2}}{z_{13}^{2} z_{24}^{2}-z_{14}^{2} z_{23}^{2}}\right] \ln \frac{z_{13}^{2} z_{24}^{2}}{z_{14}^{2} z_{23}^{2}}\right\} \\
& \times\left[2 \operatorname{tr}\left\{\hat{U}_{z_{1}}^{\eta} \hat{U}_{z_{3}}^{\dagger \eta}\right\} \operatorname{tr}\left\{\hat{U}_{z_{3}}^{\eta} \hat{U}_{z_{4}}^{\dagger \eta}\right\} \operatorname{tr}\left\{\hat{U}_{z_{4}}^{\eta} \hat{U}_{z_{2}}^{\dagger \eta}\right\}\right. \\
&\left.-\operatorname{tr}\left\{\hat{U}_{z_{1}}^{\eta} \hat{U}_{z_{3}}^{\dagger \eta} \hat{U}_{z_{4}}^{\eta} U_{z_{2}}^{\dagger \eta} \hat{U}_{z_{3}}^{\eta} \hat{U}_{z_{4}}^{\dagger \eta}\right\}-\operatorname{tr}\left\{\hat{U}_{z_{1}}^{\eta} \hat{U}_{z_{4}}^{\dagger \eta} \hat{U}_{z_{3}}^{\eta} U_{z_{2}}^{\dagger \eta} \hat{U}_{z_{4}}^{\eta} \hat{U}_{z_{3}}^{\dagger \eta}\right\}-\left(z_{4} \rightarrow z_{3}\right)\right] .
\end{aligned}
$$

Note that it can be obtained from the equation in the adjoint representation (51) by same replacement $T^{a} \rightarrow t^{a}, \operatorname{Tr} \rightarrow \operatorname{tr}$. 


\subsection{In $Q C D$}

It is instructive also to present the evolution equation for composite operator (64) in QCD. The resulting equation will not be Möbius-invariant because of the running coupling constant so composite operators (64) and (65) are not strictly speaking conformal. We will, however, keep the notation $[\cdots]^{\text {conf }}$ as a reminder that these operators were conformal in $\mathcal{N}=4$ SYM.

To get the evolution equation for "conformal" composite operators (64) in QCD we subtract the scalar (60) and gluino (61) contributions from Eq. (66) and add the quark contribution calculated in Refs. [17,18]. We obtain

$$
\begin{aligned}
& \frac{d}{d \eta}\left[\operatorname{tr}\left\{\hat{U}_{z_{1}}^{\eta} \hat{U}_{z_{2}}^{\dagger \eta}\right\}\right]^{\mathrm{conf}} \\
& =\frac{\alpha_{s}}{\pi^{2}} \int d^{2} z_{3} \frac{z_{12}^{2}}{z_{13}^{2} z_{23}^{2}}\left[1+\frac{\alpha_{s}}{\pi}\left[b\left(\ln \frac{z_{12}^{2} \mu^{2}}{4}+2 C\right)-b \frac{z_{13}^{2}-z_{23}^{2}}{z_{12}^{2}} \ln \frac{z_{13}^{2}}{z_{23}^{2}}\right.\right. \\
& \left.\left.+\left(\frac{67}{9}-\frac{\pi^{2}}{3}\right) N_{c}-\frac{10}{9} n_{f}\right]\left[\operatorname{tr}\left\{\hat{U}_{z_{1}}^{\eta} \hat{U}_{z_{3}}^{\dagger \eta}\right\} \operatorname{tr}\left\{\hat{U}_{z_{3}}^{\eta} \hat{U}_{z_{2}}^{\dagger \eta}\right\}-N_{c} \operatorname{tr}\left\{\hat{U}_{z_{1}}^{\eta} \hat{U}_{z_{2}}^{\dagger \eta}\right\}\right]^{\mathrm{conf}}\right] \\
& +\frac{\alpha_{s}^{2}}{16 \pi^{4}} \int \frac{d^{2} z_{3} d^{2} z_{4}}{z_{34}^{4}}\left[\left\{\left(-2+2 \frac{z_{12}^{2} z_{34}^{2}}{z_{13}^{2} z_{24}^{2}} \ln \frac{z_{12}^{2} z_{34}^{2}}{z_{14}^{2} z_{23}^{2}}\right.\right.\right. \\
& \left.\left.+\left[\frac{z_{12}^{2} z_{34}^{2}}{z_{13}^{2} z_{24}^{2}}\left(1+\frac{z_{12}^{2} z_{34}^{2}}{z_{13}^{2} z_{24}^{2}-z_{14}^{2} z_{23}^{2}}\right)+\frac{2 z_{13}^{2} z_{24}^{2}-4 z_{12}^{2} z_{34}^{2}}{z_{13}^{2} z_{24}^{2}-z_{14}^{2} z_{23}^{2}}\right] \ln \frac{z_{13}^{2} z_{24}^{2}}{z_{14}^{2} z_{23}^{2}}\right)+\left(z_{3} \leftrightarrow z_{4}\right)\right\} \\
& \times\left[\left(\operatorname{tr}\left\{\hat{U}_{z_{1}}^{\eta} \hat{U}_{z_{3}}^{\dagger \eta}\right\} \operatorname{tr}\left\{\hat{U}_{z_{3}}^{\eta} \hat{U}_{z_{4}}^{\dagger \eta}\right\} \operatorname{tr}\left\{\hat{U}_{z_{4}}^{\eta} \hat{U}_{z_{2}}^{\dagger \eta}\right\}-\operatorname{tr}\left\{\hat{U}_{z_{1}}^{\eta} \hat{U}_{z_{3}}^{\dagger \eta} \hat{U}_{z_{4}}^{\eta} \hat{U}_{z_{2}}^{\dagger \eta} \hat{U}_{z_{3}}^{\eta} \hat{U}_{z_{4}}^{\dagger \eta}\right\}\right)-\left(z_{4} \rightarrow z_{3}\right)\right] \\
& +\frac{z_{12}^{2} z_{34}^{2}}{z_{13}^{2} z_{24}^{2}}\left\{2 \ln \frac{z_{12}^{2} z_{34}^{2}}{z_{14}^{2} z_{23}^{2}}+\left[1+\frac{z_{12}^{2} z_{34}^{2}}{z_{13}^{2} z_{24}^{2}-z_{14}^{2} z_{23}^{2}}\right] \ln \frac{z_{13}^{2} z_{24}^{2}}{z_{14}^{2} z_{23}^{2}}\right\} \\
& \left.\times\left(\operatorname{tr}\left\{\hat{U}_{z_{1}}^{\eta} \hat{U}_{z_{3}}^{\dagger \eta}\right\} \operatorname{tr}\left\{\hat{U}_{z_{3}}^{\eta} \hat{U}_{z_{4}}^{\dagger \eta}\right\} \operatorname{tr}\left\{\hat{U}_{z_{4}}^{\eta} \hat{U}_{z_{2}}^{\dagger \eta}\right\}-z_{3} \leftrightarrow z_{4}\right)\right] \\
& +\frac{\alpha_{s}^{2} n_{f}}{2 \pi^{4}} \int \frac{d^{2} z_{3} d^{2} z_{4}}{z_{34}^{4}}\left\{2-\frac{z_{13}^{2} z_{24}^{2}+z_{23}^{2} z_{14}^{2}-z_{12}^{2} z_{34}^{2}}{z_{13}^{2} z_{24}^{2}-z_{14}^{2} z_{23}^{2}} \ln \frac{z_{13}^{2} z_{24}^{2}}{z_{14}^{2} z_{23}^{2}}\right\} \\
& \times \operatorname{tr}\left\{t^{a} \hat{U}_{z_{1}}^{\eta} t^{b} \hat{U}_{z_{2}}^{\dagger \eta}\right\} \operatorname{tr}\left\{t^{a} \hat{U}_{z_{3}}^{\eta} t^{b}\left(\hat{U}_{z_{4}}^{\dagger \eta}-\hat{U}_{z_{3}}^{\eta}\right)\right\}
\end{aligned}
$$

where $b=\frac{11}{3} N_{c}-\frac{2}{3} n_{f}$ and we have $\frac{67}{9}$ instead of $\frac{64}{9}$ because in QCD we use dimensional regularization rather than dimensional reduction scheme. Following the analysis of Ref. [11] we will outline how the above kernel reproduces the NLO BFKL eigenvalues [20].

In the two-gluon approximation the conformal dipoles (64) reduces to

$$
\begin{aligned}
\hat{\mathcal{U}}_{\text {conf }}^{\eta}\left(z_{1}, z_{2}\right)= & \hat{\mathcal{U}}^{\eta}\left(z_{1}, z_{2}\right) \\
& -\frac{\alpha_{s} N_{c}}{4 \pi^{2}} \int d^{2} z_{3} \frac{z_{12}^{2}}{z_{13}^{2} z_{23}^{2}} \ln \frac{a z_{12}^{2}}{z_{13}^{2} z_{23}^{2}}\left[\hat{\mathcal{U}}^{\eta}\left(z_{1}, z_{3}\right)+\hat{\mathcal{U}}^{\eta}\left(z_{2}, z_{3}\right)-\hat{\mathcal{U}}^{\eta}\left(z_{1}, z_{2}\right)\right] .
\end{aligned}
$$

Using Eq. (108) it is easy to demonstrate that (cf. Eq. (55))

$$
\begin{aligned}
& {\left[\operatorname{tr}\left\{\hat{U}_{z_{1}}^{\eta} \hat{U}_{z_{3}}^{\dagger \eta}\right\} \operatorname{tr}\left\{\hat{U}_{z_{3}}^{\eta} \hat{U}_{z_{2}}^{\dagger \eta}\right\}-N_{c} \operatorname{tr}\left\{\hat{U}_{z_{1}}^{\eta} \hat{U}_{z_{2}}^{\dagger \eta}\right\}\right]^{\operatorname{conf}}} \\
& \quad=-N_{c}\left[\hat{\mathcal{U}}_{\text {conf }}^{\eta}\left(z_{1}, z_{3}\right)+\hat{\mathcal{U}}_{\text {conf }}^{\eta}\left(z_{2}, z_{3}\right)-\hat{\mathcal{U}}_{\text {conf }}^{\eta}\left(z_{1}, z_{1}\right)\right],
\end{aligned}
$$


and therefore the evolution equation (67) turns into

$$
\begin{aligned}
\frac{d}{d \eta} \hat{\mathcal{U}}_{\mathrm{conf}}^{\eta}\left(z_{1}, z_{2}\right) & \\
= & \frac{\alpha_{s} N_{c}}{2 \pi^{2}} \int d^{2} z_{3} \frac{z_{12}^{2}}{z_{13}^{2} z_{23}^{2}}\left[1+\frac{\alpha_{s}}{4 \pi}\left[b\left(\frac{\ln z_{12}^{2} \mu^{2}}{4}+2 C\right)-b \frac{z_{13}^{2}-z_{23}^{2}}{z_{12}^{2}} \ln \frac{z_{13}^{2}}{z_{23}^{2}}\right.\right. \\
& \left.\left.+\left(\frac{67}{9}-\frac{\pi^{2}}{3}\right) N_{c}-\frac{10}{9} n_{f}\right]\left[\hat{\mathcal{U}}_{\mathrm{conf}}^{\eta}\left(z_{1}, z_{3}\right)+\hat{\mathcal{U}}_{\mathrm{conf}}^{\eta}\left(z_{2}, z_{3}\right)-\hat{\mathcal{U}}_{\mathrm{conf}}^{\eta}\left(z_{1}, z_{2}\right)\right]\right] \\
& +\frac{\alpha_{s}^{2} N_{c}^{2}}{8 \pi^{4}} \int \frac{d^{2} z_{3} d^{2} z_{4}}{z_{34}^{2}}\left\{2 \frac{z_{12}^{2} z_{34}^{2}}{z_{13}^{2} z_{24}^{2}} \ln \frac{z_{12}^{2} z_{34}^{2}}{z_{14}^{2} z_{23}^{2}}+\frac{z_{12}^{2} z_{34}^{2}}{z_{13}^{2} z_{24}^{2}}\left(1+\frac{z_{12}^{2} z_{34}^{2}}{z_{13}^{2} z_{24}^{2}-z_{14}^{2} z_{23}^{2}}\right) \ln \frac{z_{13}^{2} z_{24}^{2}}{z_{14}^{2} z_{23}^{2}}\right. \\
& -\frac{3 z_{12}^{2} z_{34}^{2}}{z_{13}^{2} z_{24}^{2}-z_{14}^{2} z_{23}^{2}} \ln \frac{z_{13}^{2} z_{24}^{2}}{z_{14}^{2} z_{23}^{2}} \\
& \left.+\left(1+\frac{n_{f}}{N_{c}^{3}}\right)\left(\frac{z_{13}^{2} z_{24}^{2}+z_{14}^{2} z_{23}^{2}-z_{12}^{2} z_{34}^{2}}{z_{13}^{2} z_{24}^{2}-z_{14}^{2} z_{23}^{2}} \ln \frac{z_{13}^{2} z_{24}^{2}}{z_{14}^{2} z_{23}^{2}}-2\right)\right\} \hat{\mathcal{U}}_{\mathrm{conf}}^{\eta}\left(z_{3}, z_{4}\right) \\
& +\frac{3 \alpha_{s}^{2} N_{c}^{2}}{2 \pi^{2}} \zeta(3) \hat{\mathcal{U}}^{\eta}\left(z_{1}, z_{2}\right),
\end{aligned}
$$

where we used formula

$$
\begin{aligned}
& \int d^{2} z_{4}\left\{\frac{z_{12}^{2}}{z_{13}^{2} z_{24}^{2} z_{34}^{2}}\left(2 \ln \frac{z_{12}^{2} z_{34}^{2}}{z_{14}^{2} z_{23}^{2}}+\left[1+\frac{z_{12}^{2} z_{34}^{2}}{z_{13}^{2} z_{24}^{2}-z_{14}^{2} z_{23}^{2}}\right] \ln \frac{z_{13}^{2} z_{24}^{2}}{z_{14}^{2} z_{23}^{2}}\right)-z_{3} \leftrightarrow z_{4}\right\} \\
& =12 \pi \zeta(3)\left[\delta\left(z_{23}\right)-\delta\left(z_{13}\right)\right],
\end{aligned}
$$

following from integrals (114) and (125) from Appendix E.

For the case of forward scattering $\langle\hat{\mathcal{U}}(x, y)\rangle=\mathcal{U}(x-y)$ and the linearized equation (70) can be reduced to an integral equation with respect to one variable $z \equiv z_{12}$. Using integrals (104)(106) from Ref. [11] and the integral

$$
\int d \tilde{z} \frac{1}{\tilde{z}^{2}\left(z-z^{\prime}-\tilde{z}\right)^{2}} \ln \frac{z^{2} z^{\prime 2}}{\left(z-\tilde{z}^{2}\right)\left(z^{\prime}-\tilde{z}^{2}\right)}=-\frac{\pi}{\left(z-z^{\prime}\right)^{2}} \ln ^{2} \frac{z^{2}}{z^{\prime 2}}
$$

we obtain

$$
\begin{aligned}
\frac{d}{d \eta} \hat{\mathcal{U}}(z)= & \frac{\alpha_{s} N_{c}}{2 \pi^{2}} \int d^{2} z \frac{z^{2}}{\left(z-z^{\prime}\right)^{2} z^{\prime 2}}\left\{1+\frac{\alpha_{s}}{4 \pi}\left[b \ln z^{2} \mu^{2}-b \frac{\left(z-z^{\prime}\right)^{2}-z^{\prime 2}}{z^{2}} \ln \frac{\left(z-z^{\prime}\right)^{2}}{z^{\prime 2}}\right.\right. \\
& \left.\left.+\left(\frac{67}{9}-\frac{\pi^{2}}{3}\right) N_{c}-\frac{10}{9} n_{f}\right]\left[\left\langle\hat{\mathcal{U}}\left(z-z^{\prime}\right)\right\rangle+\left\langle\hat{\mathcal{U}}\left(z^{\prime}\right)\right\rangle-\langle\hat{\mathcal{U}}(z)\rangle\right]\right\} \\
& +\frac{\alpha_{s}^{2} N_{c}^{2}}{4 \pi^{3}} \int d^{2} z^{\prime} \frac{z^{2}}{z^{\prime 2}}\left[-\frac{1}{\left(z-z^{\prime}\right)^{2}} \ln ^{2} \frac{z^{2}}{z^{\prime 2}}+F\left(z, z^{\prime}\right)+\Phi\left(z, z^{\prime}\right)\right] \mathcal{U}\left(z^{\prime}\right) \\
& +3 \frac{\alpha_{s}^{2} N_{c}^{2}}{2 \pi^{2}} \zeta(3) \mathcal{U}(z),
\end{aligned}
$$

where

$$
F\left(z, z^{\prime}\right)=\left(1+\frac{n_{f}}{N_{c}^{3}}\right) \frac{3\left(z, z^{\prime}\right)^{2}-2 z^{2} z^{\prime 2}}{16 z^{2} z^{\prime 2}}\left(\frac{2}{z^{2}}+\frac{2}{z^{\prime 2}}+\frac{z^{2}-z^{\prime 2}}{z^{2} z^{\prime 2}} \ln \frac{z^{2}}{z^{\prime 2}}\right)
$$




$$
\begin{aligned}
& -\left[3+\left(1+\frac{n_{f}}{N_{c}^{3}}\right)\left(1-\frac{\left(z^{2}+z^{\prime 2}\right)^{2}}{8 z^{2} z^{\prime 2}}+\frac{3 z^{4}+3 z^{\prime 4}-2 z^{2} z^{\prime 2}}{16 z^{4} z^{\prime 4}}\left(z, z^{\prime}\right)^{2}\right)\right] \\
& \times \int_{0}^{\infty} d t \frac{1}{z^{2}+t^{2} z^{\prime 2}} \ln \frac{1+t}{|1-t|}
\end{aligned}
$$

and

$$
\begin{aligned}
\Phi\left(z, z^{\prime}\right)= & \frac{\left(z^{2}-z^{\prime 2}\right)}{\left(z-z^{\prime}\right)^{2}\left(z+z^{\prime}\right)^{2}}\left[\ln \frac{z^{2}}{z^{\prime 2}} \ln \frac{z^{2} z^{\prime 2}\left(z-z^{\prime}\right)^{4}}{\left(z^{2}+z^{\prime 2}\right)^{4}}+2 \operatorname{Li}_{2}\left(-\frac{z^{\prime 2}}{z^{2}}\right)-2 \operatorname{Li}_{2}\left(-\frac{z^{2}}{z^{\prime 2}}\right)\right] \\
& -\left(1-\frac{\left(z^{2}-z^{\prime 2}\right)^{2}}{\left(z-z^{\prime}\right)^{2}\left(z+z^{\prime}\right)^{2}}\right)\left[\int_{0}^{1}-\int_{1}^{\infty}\right] \frac{d u}{\left(z-z^{\prime} u\right)^{2}} \ln \frac{u^{2} z^{\prime 2}}{z^{2}} .
\end{aligned}
$$

The function $-\frac{1}{\left(q-q^{\prime}\right)^{2}} \ln ^{2} \frac{q^{2}}{q^{\prime 2}}+F\left(q, q^{\prime}\right)+\Phi\left(q, q^{\prime}\right)$ enters the NLO BFKL equation in the momentum space [20] and since the eigenfunctions of the forward BFKL equation are powers both in the coordinate and momentum space, it is clear that the corresponding eigenvalues coincide. As to the first term in the r.h.s. of Eq. (72), one can demonstrate using the analysis carried out in Ref. [11] that this term also agrees with the eigenvalues of Ref. [20]. However, this analysis would lead us away from the main topic of this paper so we defer it until our next publication. It should be also mentioned that the statement in our previous paper [11] that our Eq. (59) disagrees with NLO BFKL was due to erroneous calculation of the integral (114) which was assumed to be zero. After taking into account the $\delta$-function contributions in the r.h.s. of Eq. (114) the disagreement disappears.

\section{Conclusion}

The amplitudes in $N=4$ SYM are conformally invariant and therefore the Regge limit (81) of these amplitudes must be invariant with respect to Möbius transformations of the transverse plane. If we want to use the operator expansion to find this amplitude, it is better to expand in operators which are Möbius-invariant. As we demonstrate in Appendix A, the light-like Wilson lines are formally invariant. However, they are divergent in the longitudinal direction, and at present the regularization of this rapidity divergence which respects the conformal invariance is not known. We manage to circumvent this problem by using the non-invariant "rigid cutoff" (8) and restoring the conformal invariance order by order in perturbation theory by subtracting the proper counterterms (made again of Wilson lines). The resulting NLO evolution equation for "composite conformal dipoles" is Möbius-invariant and agrees with forward NLO BFKL calculations in $\mathcal{N}=4 \mathrm{SYM}$.

Let us comment on the non-conformal result of the calculation of NLO BFKL kernel in $\mathcal{N}=4$ SYM carried out in Ref. [21]. We think that the difference between our kernel and that of Ref. [21] is due to different cutoffs for longitudinal integrations. The authors of Ref. [21] propose that the transformation of their kernel of the type $\hat{K}_{\mathrm{NLO}} \rightarrow \hat{K}_{\mathrm{NLO}}-\alpha_{s}\left[\hat{K}_{\mathrm{LO}}\right.$, $\left.\hat{\mathcal{O}}\right]$ with some suitable operator $\hat{\mathcal{O}}$ will restore conformal invariance. This is exactly what happens in our case of the kernel (44) with the "rigid cutoff" (8) of the rapidity divergence. Let us discuss the transformation proposed in Ref. [21] in our language. If we define $\tilde{\hat{\mathcal{V}}}(z)$ as

$$
\hat{\tilde{\mathcal{V}}}^{\eta}(z)=\hat{\mathcal{V}}^{\eta}(z)+\alpha_{s} \int d^{2} z^{\prime} f\left(z, z^{\prime}\right) \hat{\mathcal{V}}^{\eta}\left(z^{\prime}\right)
$$


we see that the (linear) evolution equations for the operators $\hat{\mathcal{V}}^{\eta}$ and $\hat{\tilde{\mathcal{V}}}^{\eta}$ are related by

$$
\hat{\tilde{K}}_{\mathrm{NLO}}=\hat{K}_{\mathrm{NLO}}-\alpha_{s}\left[\hat{K}_{\mathrm{LO}}, \hat{f}\right] .
$$

Indeed, differentiating the operator (73) with respect to $\eta$ we obtain

$$
\begin{aligned}
\frac{d}{d \eta} \hat{\tilde{V}}^{\eta}(z)= & \frac{d}{d \eta} \hat{\mathcal{V}}^{\eta}(z)+\alpha_{s} \int d^{2} z^{\prime} f\left(z, z^{\prime}\right) \frac{d}{d \eta} \hat{\mathcal{V}}^{\eta}\left(z^{\prime}\right) \\
= & \int d z^{\prime} K_{\mathrm{LO}}\left(z, z^{\prime}\right) \hat{\mathcal{V}}^{\eta}\left(z^{\prime}\right)+\alpha_{s} \int d z^{\prime} K_{\mathrm{NLO}}\left(z, z^{\prime}\right) \hat{\mathcal{V}}^{\eta}\left(z^{\prime}\right) \\
& +\alpha_{s} \int d z^{\prime} d z^{\prime \prime} f\left(z, z^{\prime}\right) K_{\mathrm{LO}}\left(z^{\prime}, z^{\prime \prime}\right) \hat{\mathcal{V}}^{\eta}\left(z^{\prime}\right) \\
= & \int d z^{\prime} K_{\mathrm{LO}}\left(z, z^{\prime}\right) \hat{\tilde{\mathcal{V}}}^{\eta}(z)+\alpha_{s} \int d z^{\prime} K_{\mathrm{NLO}}\left(z, z^{\prime}\right) \hat{\tilde{\mathcal{V}}}^{\eta}\left(z^{\prime}\right) \\
& +\alpha_{s} \int d z^{\prime} d z^{\prime \prime}\left[f\left(z, z^{\prime}\right) K_{\mathrm{LO}}\left(z^{\prime}, z^{\prime \prime}\right)-K_{\mathrm{LO}}\left(z, z^{\prime}\right) F\left(z^{\prime}, z^{\prime \prime}\right)\right] \hat{\tilde{\mathcal{V}}}^{\eta}(z) \\
& +O\left(\alpha_{s}^{3}\right),
\end{aligned}
$$

which corresponds to Eq. (74). Our transition between $\hat{\tilde{\mathcal{V}}}^{\eta}$ and $\hat{\tilde{\mathcal{V}}}_{\text {conf }}^{\eta}$ is of the type (73) so it is not surprising that the kernels (44) and (6) are different. We think that one can recover the conformal kernel (6) from the kernel of Ref. [21] as long as one finds the appropriate $f\left(z, z^{\prime}\right)$. It should be also mentioned that the transformation (73) with both $\hat{\mathcal{V}}^{\eta}$ and $f\left(z, z^{\prime}\right)$ conformally invariant does not change $K_{\mathrm{NLO}}$ as can be easily seen from Eq. (75). Thus, the form of the conformal composite dipole is not unique (our Eq. (7) is one particular choice) but the conformal kernel $\hat{K}_{\text {NLO }}$ is unique.

In conclusion let us discuss possible generalizations of our method. The operator expansion of the type (82) is relevant for processes like deep inelastic scattering where the strong gluon fields come from the nucleon (or nucleus) target and the spectator (virtual photon) is a weak source of the gluon field. For the processes like heavy-ion collisions, the projectile-target symmetric language of $(2+1)$-dimensional effective action seems more adequate (here 2 is the number of transverse dimensions and 1 stands for rapidity). There are many attempts in the literature to find comprehensive effective action [22] but the answer for the ultimate high-energy effective action eludes us so far. It is possible that considering this problem in $\mathcal{N}=4 \mathrm{SYM}$ where we have the additional requirement of conformal (Möbius) symmetry to restrict the effective action will help us to find the correct effective action at high energies.

\section{Acknowledgements}

The authors are grateful to L.N. Lipatov and J. Penedones for valuable discussions. This work was supported by contract DE-AC05-06OR23177 under which the Jefferson Science Associates, LLC operate the Thomas Jefferson National Accelerator Facility.

\section{Appendix A. Conformal properties of the light-like Wilson lines}

In this Section we demonstrate that the light-like Wilson lines are invariant under the conformal (Möbius) group $S L(2, C)$. It is easy to demonstrate that the set of transverse-space operators 


$$
\begin{array}{lll}
\hat{S}_{-} \equiv \frac{i}{2}\left(K^{1}+i K^{2}\right), & \hat{S}_{0} \equiv \frac{i}{2}\left(D+i M^{12}\right), & \hat{S}_{+} \equiv \frac{i}{2}\left(P^{1}-i P^{2}\right), \\
\overline{\hat{S}}_{-} \equiv \frac{i}{2}\left(K^{1}-i K^{2}\right), & \overline{\hat{S}}_{0} \equiv \frac{i}{2}\left(D-i M^{12}\right), & \overline{\hat{S}}_{+} \equiv \frac{i}{2}\left(P^{1}+i P^{2}\right),
\end{array}
$$

form an $\operatorname{SL}(2, C)$ algebra:

$$
\begin{array}{ll}
{\left[\hat{S}_{0}, \hat{S}_{ \pm}\right]= \pm \hat{S}_{ \pm},} & {\left[\hat{S}_{+}, \hat{S}_{-}\right]=2 \hat{S}_{0}} \\
{\left[\overline{\hat{S}}_{0}, \overline{\hat{S}}_{ \pm}\right]= \pm \overline{\hat{S}}_{ \pm},} & {\left[\overline{\hat{S}}_{+}, \overline{\hat{S}}_{-}\right]=2 \overline{\hat{S}}_{0}}
\end{array}
$$

Here we use standard textbook definitions of the momentum operator $\hat{P}$, angular momentum operator $\hat{M}$, dilatation operator $\hat{D}$, and special conformal generator $\hat{K}$. Using the conventional commutators of these generators with the gluon field

$$
\begin{aligned}
& i\left[\hat{P}^{\mu}, \hat{A}^{\alpha}\right]=\partial^{\mu} \hat{A}^{\alpha}, \quad i\left[\hat{D}, \hat{A}^{\alpha}\right]=\left(x_{\mu} \partial^{\mu}+1\right) \hat{A}^{\alpha}, \\
& i\left[\hat{M}^{\mu \nu}, \hat{A}^{\alpha}\right]=\left(x^{\mu} \partial^{\nu}-x^{\nu} \partial^{\mu}\right) \hat{A}^{\alpha}-\left(g^{\nu \alpha} \hat{A}^{\mu}-g^{\mu \alpha} \hat{A}^{\nu}\right), \\
& i\left[K^{\mu}, A^{\alpha}\right]=\left(2 x^{\mu} x_{\nu} \partial^{\nu}-x^{2} \partial^{\mu}+2 x^{\mu}\right) A^{\alpha}-2 x_{v}\left(g^{v \alpha} A^{\mu}-g^{\mu \alpha} A^{\nu}\right),
\end{aligned}
$$

one can easily obtain the action of these operators on the light-like Wilson lines. In complex notations

$$
\begin{aligned}
& z \equiv z^{1}+i z^{2}, \quad \bar{z} \equiv z^{1}-i z^{2}, \\
& \frac{\partial}{\partial z}=\frac{1}{2}\left(\frac{\partial}{\partial z^{1}}-i \frac{\partial}{\partial z^{2}}\right), \quad \frac{\partial}{\partial \bar{z}}=\frac{1}{2}\left(\frac{\partial}{\partial z^{1}}+i \frac{\partial}{\partial z^{2}}\right),
\end{aligned}
$$

these commutators take the form

$$
\begin{array}{ll}
{\left[\hat{S}_{-}, \hat{U}(z, \bar{z})\right]=z^{2} \partial_{z} \hat{U}(z, \bar{z}),} & {\left[\hat{S}_{0}, \hat{U}(z, \bar{z})\right]=z \partial_{z} \hat{U}(z, \bar{z}),} \\
{\left[\hat{S}_{+}, \hat{U}(z, \bar{z})\right]=-\partial_{z} \hat{U}(z, \bar{z}),} & {\left[\overline{\hat{S}}_{-}, \hat{U}(z, \bar{z})\right]=\bar{z}^{2} \partial_{\bar{z}} \hat{U}(z, \bar{z}),} \\
{\left[\overline{\hat{S}}_{0}, \hat{U}(z, \bar{z})\right]=\bar{z} \partial_{\bar{z}} \hat{U}(z, \bar{z}),} & {\left[\overline{\hat{S}}_{+}, \hat{U}(z, \bar{z})\right]=-\partial_{\bar{z}} \hat{U}(z, \bar{z}) .}
\end{array}
$$

These equations mean that the operators $U(z, \bar{z})$ lie in the standard representation of conformal group $\operatorname{SL}(2, C)$ with the conformal spin 0 .

\section{Appendix B. NLO impact factor}

As we demonstrated in Appendix A the light-like Wilson lines $U\left(x_{\perp}\right)$ are formally Möbiusinvariant and this is why the leading-order BK equation is conformal. However, because our cutoff of the rapidity divergence is not invariant, the NLO evolution kernel (44) has the non-invariant double-log term. To illustrate the non-invariance of the dipole with the cutoff (8) let us consider the high-energy operator expansion [5] of two BPS-protected currents $\mathcal{O} \equiv \frac{4 \pi^{2} \sqrt{2}}{\sqrt{N_{c}^{2}-1}} \operatorname{Tr}\left\{Z^{2}\right\}$ $\left(Z=\frac{1}{\sqrt{2}}\left(\phi_{1}+i \phi_{2}\right)\right)$ in color dipoles. The Regge limit of the amplitude

$$
A\left(x, y ; x^{\prime}, y^{\prime}\right)=(x-y)^{4}\left(x^{\prime}-y^{\prime}\right)^{4}\left\langle\mathcal{O}(x) \mathcal{O}(y) \mathcal{O}\left(x^{\prime}\right) \mathcal{O}\left(y^{\prime}\right)\right\rangle
$$

can be achieved by the rescaling [5]

$$
x \rightarrow \lambda x_{*} p_{1}+\frac{1}{\lambda} x_{\bullet} p_{2}+x_{\perp}, \quad y \rightarrow \lambda y_{*}+\frac{1}{\lambda} y_{\bullet} p_{2}+y_{\perp}
$$




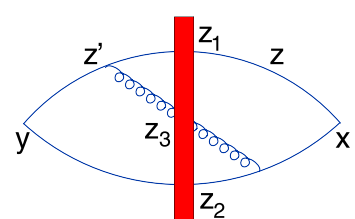

(a)

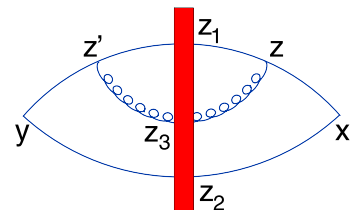

(b)

Fig. 7. Diagrams for the NLO impact factor.

with $\lambda \rightarrow \infty$. In this regime, the T-product of the currents $\mathcal{O}(x)$ and $\mathcal{O}(y)$ can be expanded in color dipoles as follows:

$$
\begin{aligned}
T\{\hat{\mathcal{O}}(x) \hat{\mathcal{O}}(y)\}= & \int d^{2} z_{1} d^{2} z_{2} I^{\mathrm{LO}}\left(z_{1}, z_{2}\right) \operatorname{Tr}\left\{\hat{U}_{z_{1}}^{\eta} \hat{U}_{z_{2}}^{\dagger \eta}\right\} \\
& +\int d^{2} z_{1} d^{2} z_{2} d^{2} z_{3} I^{\mathrm{NLO}}\left(z_{1}, z_{2}, z_{3}\right)\left[\operatorname{Tr}\left\{T^{n} \hat{U}_{z_{1}}^{\eta} \hat{U}_{z_{3}}^{\dagger \eta} T^{n} \hat{U}_{z_{3}}^{\eta} \hat{U}_{z_{2}}^{\dagger \eta}\right\}\right. \\
& \left.-N_{c} \operatorname{Tr}\left\{\hat{U}_{z_{1}}^{\eta} \hat{U}_{z_{2}}^{\dagger \eta}\right\}\right]
\end{aligned}
$$

(structure of the NLO contribution is clear from the topology of diagrams in the shock-wave background, see Fig. 7).

Let us calculate the impact factor taking $x_{\bullet}=y_{\bullet}=0$ for simplicity. The leading-order impact factor is proportional to the product of two propagators (85)

$$
\begin{aligned}
&\left\langle\left. T\{\hat{\mathcal{O}}(x) \hat{\mathcal{O}}(y)\}\right|_{A} ^{L O}\right. \\
&=\frac{16 \pi^{4}}{\left(N_{c}^{2}-1\right)} \int d^{4} z_{1} d^{4} z_{2} \delta\left(z_{1 *}\right) \delta\left(z_{2 *}\right) U_{z_{1}}^{a b} U_{z_{2}}^{a b} \\
& \quad \times\left(\frac{1}{4 \pi^{2}\left[\left(x-z_{1}\right)^{2}-i \epsilon\right]}(2 i) \partial_{*}^{\left(z_{1}\right)} \frac{1}{4 \pi^{2}\left[\left(y-z_{1}\right)^{2}-i \epsilon\right]}\right) \\
& \quad \times\left(\frac{1}{4 \pi^{2}\left[\left(x-z_{2}\right)^{2}-i \epsilon\right]}(2 i) \partial_{*}^{\left(z_{2}\right)} \frac{1}{4 \pi^{2}\left[\left(y-z_{2}\right)^{2}-i \epsilon\right]}\right) \\
&= \frac{1}{\pi^{2}\left(N_{c}^{2}-1\right)} \int d^{2} z_{1 \perp} d^{2} z_{2 \perp} \frac{\left(x_{*} y_{*}\right)^{-2}}{\mathcal{Z}_{1}^{2} z_{2}^{2}} \operatorname{Tr}\left\{U_{z_{1}} U_{z_{2}}^{\dagger}\right\},
\end{aligned}
$$

where $\mathcal{Z}_{i} \equiv \frac{\left(x-z_{i}\right)_{\perp}^{2}}{x_{*}}-\frac{\left(y-z_{i}\right)_{\perp}^{2}}{y_{*}}$ and the color trace is taken in the adjoint representation. This expressions coincides with the result of Ref. [23]. It is easy to see that under the inversion $x_{\perp} \rightarrow$ $x_{\perp} / x_{\perp}^{2}, x_{*} \rightarrow x_{*} / x_{\perp}^{2}$

$$
\begin{array}{ll}
x_{\perp} \rightarrow x_{\perp} / x_{\perp}^{2}, & x_{*} \rightarrow x_{*} / x_{\perp}^{2}, \\
y_{\perp} \rightarrow y_{\perp} / x_{\perp}^{2}, & y_{*} \rightarrow y_{*} / y_{\perp}^{2}, z_{i \perp} \rightarrow z_{i \perp} / z_{i \perp}^{2} .
\end{array}
$$

$\mathcal{Z}_{i}$ is transformed as $\mathcal{Z}_{i} \rightarrow z_{i}^{-4} \mathcal{Z}_{i}$ so the leading-order impact factor is invariant.

The NLO impact factor for two $Z^{2}$ currents is given by the two diagrams shown in Fig. 7. To calculate them we will use the following representation of scalar and gluon propagators in the shock-wave background (at $x_{*}>0>y_{*}$ ) 


$$
\begin{aligned}
\left\langle\hat{\Phi}_{I}(x) \hat{\Phi}_{J}(y)\right\rangle_{A} & =2 i \delta^{I J} \int d^{4} z \delta\left(z_{*}\right) \frac{1}{4 \pi^{2}\left[(x-z)^{2}-i \epsilon\right]} U_{z_{\perp}}^{a b} \partial_{*}^{(z)} \frac{1}{4 \pi^{2}\left[(z-y)^{2}-i \epsilon\right]} \\
& =-\frac{\delta_{I J}}{4 \pi^{3}} \int d^{2} z_{\perp} \frac{x_{*} y_{*} U_{z_{3}}^{a b}}{\left[\frac{4}{s}(x-y) \bullet x_{*} y_{*}-(x-z)_{\perp}^{2} y_{*}+(y-z)_{\perp}^{2} x_{*}+i \epsilon\right]^{2}},
\end{aligned}
$$

and

$$
\begin{aligned}
& \left\langle\hat{A}_{\mu}^{a}(x) \hat{A}_{v}^{b}(y)\right\rangle_{A} \\
& \quad=-\frac{i}{2} \int d^{4} z \delta\left(z_{*}\right) \frac{x_{*} g_{\mu \xi}^{\perp}-p_{2 \mu}(x-z)_{\frac{\perp}{\xi}}^{\perp}}{\pi^{2}\left[(x-z)^{2}-i \epsilon\right]^{2}} U_{z_{\perp}}^{a b} \frac{1}{\partial_{*}^{(z)}} \frac{y_{*} \delta_{v}^{\perp \xi}-p_{2 v}(y-z)_{\perp}^{\xi}}{\pi^{2}\left[(z-y)^{2}-i \epsilon\right]^{2}},
\end{aligned}
$$

where $\frac{1}{\partial_{*}}$ can be either $\frac{1}{\partial_{*}+i \epsilon}$ or $\frac{1}{\partial_{*}-i \epsilon}$ which leads to the same result after subtraction of the leading-order contribution (see below).

To calculate the next-to-leading impact factor we need the three-point scalar-scalar-gluon vertex Green function (vertex with tails):

$$
\begin{aligned}
& \int d^{4} z \theta\left(z_{*}\right)\left[\frac{1}{4 \pi^{2}\left[(x-z)^{2}-i \epsilon\right]} \stackrel{\leftrightarrow}{\partial} \frac{1}{\mu} \frac{1}{4 \pi^{2}\left[\left(z-z_{1}\right)^{2}-i \epsilon\right]}\right] \frac{z_{*} \delta_{\mu}^{\perp \xi}-p_{2 \mu}\left(z-z_{3}\right)_{\perp}^{\xi}}{2 \pi^{2}\left[\left(z-z_{3}\right)^{2}-i \epsilon\right]^{2}} \\
& =\frac{i x_{*} z_{13}^{\perp \xi}}{8 \pi^{4} z_{13}^{2}\left[\left(x-z_{1}\right)^{2}-i \epsilon\right]\left[\left(x-z_{3}\right)^{2}-i \epsilon\right]} .
\end{aligned}
$$

Since $z_{1 *}=z_{*}=0$ one can easily check that $\theta\left(z_{*}\right)$ in the 1.h.s. of this integral can be erased since the contribution from $z_{*}<0$ vanishes. After that, one easily gets Eq. (87) from the conformal integral

$$
\int d^{4} z\left[\frac{1}{(x-z)^{2}} \stackrel{\leftrightarrow}{\partial}_{\mu} \frac{1}{(z-y)^{2}}\right] \frac{z_{v}}{z^{4}}-\mu \leftrightarrow v=4 i \pi^{2} \frac{x_{\mu} y_{\nu}-x_{\nu} y_{\mu}}{x^{2} y^{2}(x-y)^{2}}
$$

which is easily calculated by inversion $z_{\mu} \rightarrow z_{\mu} / z^{2}$.

Now we are in position to calculate the contribution of the diagram in Fig. 7a:

$$
\begin{aligned}
\left\langle\left. T\{\hat{\mathcal{O}}(x) \hat{\mathcal{O}}(y)\}\right|_{A} ^{\text {Fig. 7a }}\right. & \\
= & g^{2} \frac{32 \pi^{4}}{N_{c}^{2}-1} \int d^{4} z_{1} d^{4} z_{2} d^{4} z_{3} \delta\left(z_{3 *}\right) \operatorname{Tr}\left\{T^{n} U_{z_{1}} T^{n^{\prime}} U_{z_{2}}^{\dagger}\right\} U_{z_{3}}^{n n^{\prime}} \\
& \times\left(2 i \partial_{1 *} \frac{\delta\left(z_{1 *}\right)}{4 \pi^{2}\left[\left(y-z_{1}\right)^{2}-i \epsilon\right]}\right)\left(2 i \partial_{2 *} \frac{\delta\left(z_{2} *\right)}{4 \pi^{2}\left[\left(x-z_{2}\right)^{2}-i \epsilon\right]}\right) \\
& \times \int d^{4} z\left(\frac{1}{4 \pi^{2}\left[(x-z)^{2}-i \epsilon\right]} \stackrel{\leftrightarrow}{\partial_{\mu}} \frac{\delta\left(z_{1} *\right)}{4 \pi^{2}\left[\left(z-z_{1}\right)^{2}-i \epsilon\right]}\right) \\
& \times \frac{z_{*} \theta\left(z_{*}\right)}{2 \pi^{2}\left[\left(z-z_{3}\right)^{2}-i \epsilon\right]^{2}}\left[g_{\mu \rho}^{\perp}-p_{2 \mu} \frac{\left(z-z_{3}\right)_{\perp}^{\rho}}{z_{*}}\right] \frac{2 i}{\partial_{3 *}} \\
& \times \int d^{4} z^{\prime}\left(\frac{\delta\left(z_{2} *\right)}{4 \pi^{2}\left[\left(z_{2}-z^{\prime}\right)^{2}-i \epsilon\right]} \stackrel{\leftrightarrow}{z_{\mu}^{\prime}} \frac{1}{4 \pi^{2}\left[\left(y-z^{\prime}\right)^{2}-i \epsilon\right]}\right) \\
& \times \frac{z_{*}^{\prime} \theta\left(-z_{*}^{\prime}\right)}{2 \pi^{2}\left[\left(z_{3}-z^{\prime}\right)^{2}-i \epsilon\right]^{2}}\left[g_{\nu}^{\perp \rho}-p_{2 v} \frac{\left(z^{\prime}-z_{3}\right)_{\perp}^{\rho}}{z_{*}^{\prime}}\right] .
\end{aligned}
$$


Using Eq. (87) one can reduce this equation to

$$
\begin{aligned}
\left\langle\left. T\{\hat{\mathcal{O}}(x) \hat{\mathcal{O}}(y)\}\right|_{A} ^{\text {Fig. 7a }}\right. & \\
= & -g^{2} \frac{32 i \pi^{4}}{N_{c}^{2}-1} \int d^{4} z_{1} d^{4} z_{2} d^{4} z_{3} \delta\left(z_{3 *}\right) \operatorname{Tr}\left\{T^{n} U_{z_{1}} T^{n^{\prime}} U_{z_{2}}^{\dagger}\right\} U_{z_{3}}^{n n^{\prime}} \\
& \times \frac{1}{4 \pi^{2}\left[\left(x-z_{1}\right)^{2}-i \epsilon\right]}\left(2 i \partial_{1 *} \frac{\delta\left(z_{1 *}\right)}{4 \pi^{2}\left[\left(y-z_{1}\right)^{2}-i \epsilon\right]}\right)\left(2 i \partial_{2 *} \frac{\delta\left(z_{2} *\right)}{4 \pi^{2}\left[\left(x-z_{2}\right)^{2}-i \epsilon\right]}\right) \\
& \times \frac{1}{4 \pi^{2}\left[\left(y-z_{2}\right)^{2}-i \epsilon\right]} \frac{\left(z_{13}, z_{23}\right)_{\perp}}{z_{13}^{2} z_{23}^{2}} \frac{x_{*}}{2 \pi^{2}\left[\left(x-z_{3}\right)^{2}-i \epsilon\right]} \frac{2}{\partial_{3 *}} \frac{y_{*}}{2 \pi^{2}\left[\left(y-z_{3}\right)^{2}-i \epsilon\right]} .
\end{aligned}
$$

Performing integration with respect to $z_{\bullet i}$ we get

$$
\begin{aligned}
\langle T\{\hat{\mathcal{O}}(x) \hat{\mathcal{O}}(y)\}\}_{A}^{\text {Fig. 7a }}= & \frac{2 \alpha_{s}}{\left(N_{c}^{2}-1\right) \pi^{4} x_{*}^{2} y_{*}^{2}} \int \frac{d^{2} z_{1} d^{2} z_{2}}{\mathcal{Z}_{1}^{2} \mathcal{Z}_{2}^{2}} \\
& \times \int d^{2} z_{3} \frac{\left(z_{13}, z_{23}\right)_{\perp}}{z_{13}^{2} z_{23}^{2}} \operatorname{Tr}\left\{T^{n} U_{z_{1}} U_{z_{3}}^{\dagger} T^{n} U_{z_{3}} U_{z_{2}}^{\dagger}\right\} \int_{0}^{\infty} \frac{d \alpha}{\alpha} e^{i \alpha \frac{s}{4} \mathcal{Z}_{3}}
\end{aligned}
$$

(recall that $\left.\mathcal{Z}_{i}=\frac{\left(x-z_{i}\right)_{\perp}^{2}}{x_{*}}-\frac{\left(y-z_{i}\right)_{\perp}^{2}}{y_{*}}\right)$. Similarly, one can demonstrate that the diagram shown in Fig. $7 \mathrm{~b}$ yields

$$
\begin{aligned}
& \left\langle\left. T\{\hat{\mathcal{O}}(x) \hat{\mathcal{O}}(y)\}\right|_{A} ^{\text {Fig. 7b }}\right. \\
& \quad=\frac{2 \alpha_{s}}{\left(N_{c}^{2}-1\right) \pi^{4} x_{*}^{2} y_{*}^{2}} \int \frac{d^{2} z_{1} d^{2} z_{2}}{\mathcal{Z}_{1}^{2} \mathcal{Z}_{2}^{2}} \int d^{2} z_{3} \frac{1}{z_{13}^{2}} \operatorname{Tr}\left\{T^{n} U_{z_{1}} U_{z_{3}}^{\dagger} T^{n} U_{z_{3}} U_{z_{2}}^{\dagger}\right\} \int_{0}^{\infty} \frac{d \alpha}{\alpha} e^{i \alpha \frac{s}{4} \mathcal{Z}_{3}} .
\end{aligned}
$$

Using the $z_{1} \leftrightarrow z_{2}$ symmetry in this equation one can write down the sum of Eq. (91) and Eq. (92) as

$$
\begin{aligned}
\left\langle\left. T\{\hat{\mathcal{O}}(x) \hat{\mathcal{O}}(y)\}\right|_{A} ^{\text {Fig. } 7}=\right. & \frac{\alpha_{s}}{\left(N_{c}^{2}-1\right) \pi^{4} x_{*}^{2} y_{*}^{2}} \int \frac{d^{2} z_{1} d^{2} z_{2}}{\mathcal{Z}_{1}^{2} \mathcal{Z}_{2}^{2}} \int d^{2} z_{3} \frac{z_{12}^{2}}{z_{13}^{2} z_{23}^{2}} \\
& \times \operatorname{Tr}\left\{T^{n} U_{z_{1}} U_{z_{3}}^{\dagger} T^{n} U_{z_{3}} U_{z_{2}}^{\dagger}\right\} \int_{0}^{\infty} \frac{d \alpha}{\alpha} e^{i \alpha \frac{s}{4} \mathcal{Z}_{3}} .
\end{aligned}
$$

It is convenient to rewrite this in the following form

$$
\begin{aligned}
& \left\langle\left. T\{\hat{\mathcal{O}}(x) \hat{\mathcal{O}}(y)\}\right|_{A} ^{\text {Fig. } 7}\right. \\
& =\frac{\alpha_{s}}{\left(N_{c}^{2}-1\right) \pi^{4} x_{*}^{2} y_{*}^{2}} \int \frac{d^{2} z_{1} d^{2} z_{2}}{\mathcal{Z}_{1}^{2} \mathcal{Z}_{2}^{2}} \int d^{2} z_{3} \frac{z_{12}^{2}}{z_{13}^{2} z_{23}^{2}} \\
& \quad \times\left[\operatorname{Tr}\left\{T^{n} U_{z_{1}} U_{z_{3}}^{\dagger} T^{n} U_{z_{3}} U_{z_{2}}^{\dagger}\right\}-N_{c} \operatorname{Tr}\left\{U_{z_{1}} U_{z_{2}}^{\dagger}\right\}\right] \int_{0}^{\infty} \frac{d \alpha}{\alpha} e^{i \alpha \frac{s}{4} \mathcal{Z}_{3}} \\
& \quad+\frac{2 \alpha_{s} N_{c}}{\left(N_{c}^{2}-1\right) \pi^{4} x_{*}^{2} y_{*}^{2}} \int \frac{d^{2} z_{1} d^{2} z_{2}}{\mathcal{Z}_{1}^{2} \mathcal{Z}_{2}^{2}} \int d^{2} z_{3} \frac{z_{12}^{2}}{z_{13}^{2} z_{23}^{2}} \operatorname{Tr}\left\{U_{z_{1}} U_{z_{2}}^{\dagger}\right\} \int_{0}^{\infty} \frac{d \alpha}{\alpha} e^{i \alpha \frac{s}{4} \mathcal{Z}_{3}} .
\end{aligned}
$$




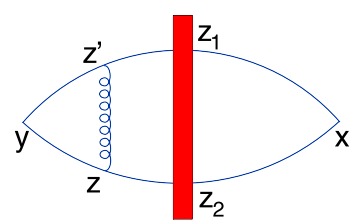

(a)

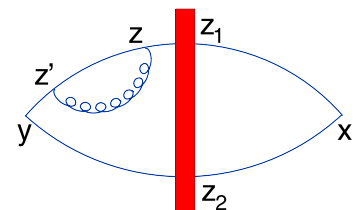

(b)

Fig. 8. Diagrams for the NLO impact factor without gluon-shockwave intersection.

Let us discuss now the contribution of Fig. 8 diagrams. Since this contribution is proportional to $\operatorname{Tr}\left\{U_{z_{1}} U_{z_{2}}^{\dagger}\right\}$ it can be restored from the comparison of Eq. (94) with the pure perturbative series for the correlator $\langle T\{\hat{\mathcal{O}}(x) \hat{\mathcal{O}}(y)\}\rangle$. Indeed, if we switch off the shock wave the contribution of Fig. 7 diagrams is given by the second term in Eq. (94) (with $U, U^{\dagger}$ replaced by 1). On the other hand, perturbative series for the correlator $\langle T\{\hat{\mathcal{O}}(x) \hat{\mathcal{O}}(y)\}\rangle$ vanishes [24] and therefore the contribution of Fig. 8 diagrams should be equal to the second term in the r.h.s. Eq. (94) with opposite sign. Thus, the first term in the r.h.s. Eq. (94) gives the total contribution to the impact factor:

$$
\begin{aligned}
& \langle T\{\hat{\mathcal{O}}(x) \hat{\mathcal{O}}(y)\}\}_{A}^{\text {Fig. 7+Fig. } 8} \\
& =\frac{\alpha_{s}}{\left(N_{c}^{2}-1\right) \pi^{4} x_{*}^{2} y_{*}^{2}} \int \frac{d^{2} z_{1} d^{2} z_{2}}{\mathcal{Z}_{1}^{2} \mathcal{Z}_{2}^{2}} \int d^{2} z_{3} \frac{z_{12}^{2}}{z_{13}^{2} z_{23}^{2}} \\
& \quad \times\left[\operatorname{Tr}\left\{T^{n} U_{z_{1}} U_{z_{3}}^{\dagger} T^{n} U_{z_{3}} U_{z_{2}}^{\dagger}\right\}-N_{c} \operatorname{Tr}\left\{U_{z_{1}} U_{z_{2}}^{\dagger}\right\}\right] \int_{0}^{\infty} \frac{d \alpha}{\alpha} e^{i \alpha \frac{s}{4} \mathcal{Z}_{3}} .
\end{aligned}
$$

The integral over $\alpha$ in the r.h.s. of Eq. (95) diverges. This divergence reflects the fact that the r.h.s. of Eq. (95) is not exactly the NLO impact factor since we must subtract the matrix element of the leading-order contribution. Indeed, the NLO impact factor is a coefficient function defined according to Eq. (82). To find the NLO impact factor, we consider the operator equation (82) in the shock-wave background (in the leading order $\left\langle\hat{U}_{z_{3}}\right\rangle_{A}=U_{z_{3}}$ ):

$$
\begin{aligned}
\langle T & \{\hat{\mathcal{O}}(x) \hat{\mathcal{O}}(y)\}\rangle_{A}-\int d^{2} z_{1} d^{2} z_{2} I^{\mathrm{LO}}\left(x, y ; z_{1}, z_{2}\right)\left\langle\operatorname{Tr}\left\{\hat{U}_{z_{1}}^{\eta} \hat{U}_{z_{2}}^{\dagger \eta}\right\}\right\rangle_{A} \\
= & \int d^{2} z_{1} d^{2} z_{2} d^{2} z_{3} I^{\mathrm{NLO}}\left(x, y ; z_{1}, z_{2}, z_{3} ; \eta\right) \\
& \times\left[\operatorname{Tr}\left\{T^{n} U_{z_{1}} U_{z_{3}}^{\dagger} T^{n} U_{z_{3}} U_{z_{2}}^{\dagger}\right\}-N_{c} \operatorname{Tr}\left\{U_{z_{1}} U_{z_{2}}^{\dagger}\right\}\right] .
\end{aligned}
$$

The NLO matrix element $\langle T\{\hat{\mathcal{O}}(x) \hat{\mathcal{O}}(y)\}\rangle_{A}$ is given by Eq. (95) while

$$
\begin{aligned}
& \int d^{2} z_{1} d^{2} z_{2} I^{\mathrm{LO}}\left(x, y ; z_{1}, z_{2}\right)\left\langle\operatorname{Tr}\left\{\hat{U}_{z_{1}}^{\eta} \hat{U}_{z_{2}}^{\dagger \eta}\right\}\right\rangle_{A} \\
& =\frac{\left(x_{*} y_{*}\right)^{-2}}{\pi^{2}\left(N_{c}^{2}-1\right)} \int d^{2} z_{1} d^{2} z_{2} \frac{1}{\mathcal{Z}_{1}^{2} \mathcal{Z}_{2}^{2}} \frac{\alpha_{s}}{\pi^{2}} \int_{0}^{\sigma} \frac{d \alpha}{\alpha} \\
& \quad \times \int d^{2} z_{3} \frac{z_{12}^{2}}{z_{13}^{2} z_{23}^{2}}\left[\operatorname{Tr}\left\{T^{n} U_{z_{1}} U_{z_{3}}^{\dagger} T^{n} U_{z_{3}} U_{z_{2}}^{\dagger}\right\}-N_{c} \operatorname{Tr}\left\{U_{z_{1}} U_{z_{2}}^{\dagger}\right\}\right],
\end{aligned}
$$


as follows from Eq. (16). The $\alpha$ integration is cut above by $\sigma=e^{\eta}$ in accordance with the definition of operators $\hat{U}^{\eta}$ (8). Subtracting (97) from Eq. (95) we get

$$
\begin{aligned}
I^{\mathrm{NLO}}\left(x, y ; z_{1}, z_{2}, z_{3} ; \eta\right) & =\frac{\alpha_{s}\left(x_{*} y_{*}\right)^{-2}}{\pi^{4}\left(N_{c}^{2}-1\right)} \frac{z_{13}^{2}}{z_{12}^{2} z_{23}^{2} \mathcal{Z}_{1}^{2} \mathcal{Z}_{2}^{2}}\left[\int_{0}^{\infty} \frac{d \alpha}{\alpha} e^{i \alpha \frac{s}{4} \mathcal{Z}_{3}}-\int_{0}^{\sigma} \frac{d \alpha}{\alpha}\right] \\
& =-\frac{\alpha_{s}\left(x_{*} y_{*}\right)^{-2}}{\pi^{4}\left(N_{c}^{2}-1\right)} \frac{z_{13}^{2}}{z_{12}^{2} z_{23}^{2} \mathcal{Z}_{1}^{2} \mathcal{Z}_{2}^{2}}\left[\ln \frac{\sigma s}{4} \mathcal{Z}_{3}-\frac{i \pi}{2}+C\right] .
\end{aligned}
$$

Let us rewrite the operator expansion (82) in the explicit form

$$
\begin{aligned}
& (x-y)^{4} T\{\hat{\mathcal{O}}(x) \hat{\mathcal{O}}(y)\} \\
& =\frac{(x-y)^{4}}{\pi^{2}\left(N_{c}^{2}-1\right)} \int d^{2} z_{1 \perp} d^{2} z_{2} \perp \frac{\left(x_{*} y_{*}\right)^{-2}}{\mathcal{Z}_{1}^{2} \mathcal{Z}_{2}^{2}} \operatorname{Tr}\left\{\hat{U}_{z_{1}}^{\eta} \hat{U}_{z_{2}}^{\dagger \eta}\right\} \\
& \quad-\frac{\alpha_{s}(x-y)^{4}}{\pi^{4}\left(N_{c}^{2}-1\right)} \int d^{2} z_{1 \perp} d^{2} z_{2} \perp d^{2} z_{3} \frac{z_{12}^{2}\left(x_{*} y_{*}\right)^{-2}}{z_{13}^{2} z_{23}^{2} \mathcal{Z}_{1}^{2} \mathcal{Z}_{2}^{2}}\left[\ln \frac{\sigma s}{4} \mathcal{Z}_{3}-\frac{i \pi}{2}+C\right] \\
& \quad \times\left[\operatorname{Tr}\left\{T^{n} \hat{U}_{z_{1}}^{\eta} \hat{U}_{z_{3}}^{\dagger \eta} T^{n} \hat{U}_{z_{3}}^{\eta} \hat{U}_{z_{2}}^{\dagger \eta}\right\}-N_{c} \operatorname{Tr}\left\{\hat{U}_{z_{1}}^{\eta} \hat{U}_{z_{2}}^{\dagger \eta}\right\}\right] .
\end{aligned}
$$

It is easy to see now that under the inversion (84) the leading-order impact factor is invariant while the NLO impact factor is not because of the non-invariant logarithmic term $\ln \frac{\sigma s}{4} \mathcal{Z}_{3}$. Since the original T-product of the currents in the 1.h.s. of Eq. (99) is conformal, it indicates that our operators $\hat{U}^{\eta}$ with the "rigid cutoff" (8) are not Möbius-invariant. However, if we expand the original T-product in composite conformal operators (7) instead, the resulting impact factor is conformally invariant:

$$
\begin{aligned}
(x-y)^{4} T\{\hat{\mathcal{O}}(x) \hat{\mathcal{O}}(y)\}= & \frac{(x-y)^{4}}{\pi^{2}\left(N_{c}^{2}-1\right)} \int d^{2} z_{1 \perp} d^{2} z_{2 \perp} \frac{\left(x_{*} y_{*}\right)^{-2}}{\mathcal{Z}_{1}^{2} \mathcal{Z}_{2}^{2}}\left[\operatorname{Tr}\left\{\hat{U}_{z_{1}}^{\eta} \hat{U}_{z_{2}}^{\dagger \eta}\right\}\right]^{\mathrm{conf}} \\
& -\frac{\alpha_{s}(x-y)^{4}}{2 \pi^{4}\left(N_{c}^{2}-1\right)} \int d^{2} z_{1 \perp} d^{2} z_{2 \perp} d^{2} z_{3} \frac{z_{12}^{2}\left(x_{*} y_{*}\right)^{-2}}{z_{13}^{2} z_{23}^{2} \mathcal{Z}_{1}^{2} \mathcal{Z}_{2}^{2}} \\
& \times\left(\ln \frac{a \sigma^{2} s^{2} z_{12}^{2}}{16 z_{13}^{2} z_{23}^{2}}\left[\frac{\left(x-z_{3}\right)^{2}}{x_{*}}-\frac{\left(y-z_{3}\right)^{2}}{y_{*}}\right]^{2}-i \pi+2 C\right) \\
& \times\left[\operatorname{Tr}\left\{T^{n} \hat{U}_{z_{1}}^{\eta} \hat{U}_{z_{3}}^{\dagger \eta} T^{n} \hat{U}_{z_{3}}^{\eta} \hat{U}_{z_{2}}^{\dagger \eta}\right\}-N_{c} \operatorname{Tr}\left\{\hat{U}_{z_{1}}^{\eta} \hat{U}_{z_{2}}^{\dagger \eta}\right\}\right] .
\end{aligned}
$$

The arbitrary dimensional constant $a$ should be chosen in such a way that the impact factor in the r.h.s. of Eq. (101) does not change under the rescaling (81). The proper choice for our T-product is $a=\frac{x_{*} y_{*}}{s^{2}(x-y)^{2}}$ so our final operator expansion takes the form

$$
\begin{aligned}
(x- & y)^{4} T\{\hat{\mathcal{O}}(x) \hat{\mathcal{O}}(y)\} \\
= & \frac{(x-y)^{4}}{\pi^{2}\left(N_{c}^{2}-1\right)} \int d^{2} z_{1} d^{2} z_{2} \frac{\left(x_{*} y_{*}\right)^{-2}}{\mathcal{Z}_{1}^{2} \mathcal{Z}_{2}^{2}}\left[\operatorname{Tr}\left\{\hat{U}_{z_{1}}^{\eta} \hat{U}_{z_{2}}^{\dagger \eta}\right\}\right]^{\mathrm{conf}} \\
& -\frac{\alpha_{s}(x-y)^{4}}{2 \pi^{4}\left(N_{c}^{2}-1\right)} \int d^{2} z_{1} d^{2} z_{2} d^{2} z_{3} \frac{z_{12}^{2}\left(x_{*} y_{*}\right)^{-2}}{z_{13}^{2} z_{23}^{2} \mathcal{Z}_{1}^{2} \mathcal{Z}_{2}^{2}}
\end{aligned}
$$




$$
\begin{aligned}
& \times\left(\ln \frac{x_{*} y_{*} z_{12}^{2} e^{2 \eta}}{16(x-y)_{\perp}^{2} z_{13}^{2} z_{23}^{2}}\left[\frac{\left(x-z_{3}\right)^{2}}{x_{*}}-\frac{\left(y-z_{3}\right)^{2}}{y_{*}}\right]^{2}-i \pi+2 C\right) \\
& \times\left[\operatorname{Tr}\left\{T^{n} \hat{U}_{z_{1}}^{\eta} \hat{U}_{z_{3}}^{\dagger \eta} T^{n} \hat{U}_{z_{3}}^{\eta} \hat{U}_{z_{2}}^{\dagger \eta}\right\}-N_{c} \operatorname{Tr}\left\{\hat{U}_{z_{1}}^{\eta} \hat{U}_{z_{2}}^{\dagger \eta}\right\}\right],
\end{aligned}
$$

where the conformal dipole $\left[\operatorname{Tr}\left\{\hat{U}_{z_{1}}^{\eta} \hat{U}_{z_{2}}^{\dagger \eta}\right\}\right]^{\text {conf }}$ is given by Eq. (7) with $a=\frac{x_{*} y_{*}}{s^{2}(x-y)^{2}}$. Now it is evident that the impact factor in the r.h.s. of this equation is Möbius-invariant and does not scale with $\lambda$ so Eq. (45) gives conformally invariant operator up to $\alpha_{s}^{2}$ order. In higher orders, one should expect the correction terms with more Wilson lines. This procedure of finding the dipole with conformally regularized rapidity divergence is analogous to the construction of the composite renormalized local operator by adding the appropriate counterterms order by order in perturbation theory.

\section{Appendix C. Leading-order evolution of the four-Wilson-line operator}

In this appendix we derive the evolution equation for the four-Wilson-line operator $\operatorname{Tr}\left\{T^{a} \hat{U}_{z_{1}}^{\eta} \hat{U}_{z_{3}}^{\dagger \eta} T^{a} \hat{U}_{z_{3}}^{\eta} \hat{U}_{z_{2}}^{\dagger \eta}\right\}$ in the leading order in perturbation theory. As a first step, we rewrite the hierarchy equations of Ref. [5] in the adjoint representation. It takes the form

$$
\begin{aligned}
& \frac{d}{d \eta}\left(\hat{U}_{z_{1}}^{\eta}\right)_{i j}\left(\hat{U}_{z_{2}}^{\eta}\right)_{k l} \\
& =\frac{\alpha_{s}}{2 \pi^{2}} \int d^{2} z_{3} \frac{\left(z_{13}, z_{23}\right)}{z_{13}^{2} z_{23}^{2}}\left[\left(T^{a} \hat{U}_{z_{1}}^{\eta}\right)_{i j}\left(\hat{U}_{z_{2}}^{\eta} T^{b}\right)_{k l}\right. \\
& \left.\quad+\left(\hat{U}_{z_{1}}^{\eta} T^{b}\right)_{i j}\left(T^{a} \hat{U}_{z_{2}}^{\eta}\right)_{k l}\right]\left(2 \hat{U}_{z_{3}}^{\eta}-\hat{U}_{z_{1}}^{\eta}-\hat{U}_{z_{2}}^{\eta}\right)^{a b},
\end{aligned}
$$

for the pair-wise interaction and

$$
\frac{d}{d \eta}\left(\hat{U}_{z_{1}}^{\eta}\right)_{i j}=\frac{\alpha_{s}}{\pi^{2}} \int d^{2} z_{3} \frac{1}{z_{13}^{2}}\left(T^{a} \hat{U}_{z_{1}}^{\eta} T^{b}\right)_{i j}\left(\hat{U}_{z_{3}}^{\eta}-\hat{U}_{z_{1}}^{\eta}\right)^{a b}
$$

for the self-interaction. Using the above equations we get

$$
\begin{aligned}
\frac{d}{d \eta}[ & \left.\operatorname{Tr}\left\{T^{n} \hat{U}_{z_{1}}^{\eta} \hat{U}_{z_{3}}^{\dagger \eta} T^{n} \hat{U}_{z_{3}}^{\eta} \hat{U}_{z_{2}}^{\dagger \eta}\right\}-N_{c} \operatorname{Tr}\left\{\hat{U}_{z_{1}}^{\eta} \hat{U}_{z_{2}}^{\dagger \eta}\right\}\right] \\
= & \frac{\alpha_{s}}{2 \pi^{2}} \int d^{2} z_{4}\left(\hat{U}_{z_{3}}^{\eta}\right)^{a a^{\prime}}\left[\frac { ( z _ { 1 4 } , z _ { 3 4 } ) } { z _ { 1 4 } ^ { 2 } z _ { 3 4 } ^ { 2 } } \operatorname { T r } \left\{T^{a} T^{b} \hat{U}_{z_{1}}^{\eta}\left[T^{a^{\prime}}, T^{b^{\prime}}\right] \hat{U}_{z_{2}}^{\dagger \eta}\right.\right. \\
& \left.+\left[T^{b}, T^{a}\right] \hat{U}_{z_{1}}^{\eta} T^{b^{\prime}} T^{a^{\prime}} \hat{U}_{z_{2}}^{\dagger \eta}\right\}\left(2 \hat{U}_{z_{4}}^{\eta}-\hat{U}_{z_{1}}^{\eta}-\hat{U}_{z_{3}}^{\eta}\right)^{b b^{\prime}} \\
& +\frac{\left(z_{24}, z_{34}\right)}{z_{24}^{2} z_{34}^{2}} \operatorname{Tr}\left\{\left[T^{a}, T^{b}\right] \hat{U}_{z_{1}}^{\eta} T^{a^{\prime}} T^{b^{\prime}} \hat{U}_{z_{2}}^{\dagger \eta}\right. \\
& \left.+T^{b} T^{a} \hat{U}_{z_{1}}^{\eta}\left[T^{b^{\prime}}, T^{a^{\prime}}\right] \hat{U}_{z_{2}}^{\dagger \eta}\right\}\left(2 \hat{U}_{z_{4}}^{\eta}-\hat{U}_{z_{2}}^{\eta}-\hat{U}_{z_{3}}^{\eta}\right)^{b b^{\prime}} \\
& -\frac{\left(z_{14}, z_{24}\right)}{z_{14}^{2} z_{24}^{2}} \operatorname{Tr}\left\{T^{a} T^{b} \hat{U}_{z_{1}}^{\eta} T^{a^{\prime}} T^{b^{\prime}} \hat{U}_{z_{2}}^{\dagger \eta}+T^{b} T^{a} \hat{U}_{z_{1}}^{\eta} T^{b^{\prime}} T^{a^{\prime}} \hat{U}_{z_{2}}^{\dagger \eta}\right\}\left(2 \hat{U}_{z_{4}}^{\eta}-\hat{U}_{z_{1}}^{\eta}-\hat{U}_{z_{2}}^{\eta}\right) \\
& -\frac{1}{z_{34}^{2}} \operatorname{Tr}\left\{\left[T^{a}, T^{b}\right] \hat{U}_{z_{1}}^{\eta}\left[T^{a^{\prime}}, T^{b^{\prime}}\right] \hat{U}_{z_{2}}^{\dagger \eta}\right\}\left(2 \hat{U}_{z_{4}}^{\eta}-2 \hat{U}_{z_{3}}^{\eta}\right)^{b b^{\prime}} \\
& +\frac{1}{z_{14}^{2}} \operatorname{Tr}\left\{T^{a} T^{b} \hat{U}_{z_{1}}^{\eta} T^{b^{\prime}} T^{a^{\prime}} \hat{U}_{z_{2}}^{\dagger \eta}\right\}\left(2 \hat{U}_{z_{4}}^{\eta}-2 \hat{U}_{z_{1}}^{\eta}\right)^{b b^{\prime}}
\end{aligned}
$$




$$
\begin{aligned}
& \left.+\frac{1}{z_{24}^{2}} \operatorname{Tr}\left\{T^{b} T^{a} \hat{U}_{z_{1}}^{\eta} T^{a^{\prime}} T^{b^{\prime}} \hat{U}_{z_{2}}^{\dagger \eta}\right\}\left(2 \hat{U}_{z_{4}}^{\eta}-2 \hat{U}_{z_{2}}^{\eta}\right)^{b b^{\prime}}\right] \\
& -\frac{\alpha_{s} N_{c}}{\pi^{2}} \int d^{2} z_{4} \frac{z_{12}^{2}}{z_{14}^{2} z_{24}^{2}}\left(\operatorname{Tr}\left\{T^{a} U_{z_{1}} U_{z_{4}}^{\dagger} T^{a} U_{z_{4}} U_{z_{2}}^{\dagger}\right\}-N_{c} \operatorname{Tr}\left\{U_{z_{1}} U_{z_{2}}^{\dagger}\right\}\right) .
\end{aligned}
$$

After some algebra, the r.h.s. of Eq. (104) reduces to

$$
\begin{aligned}
\frac{d}{d \eta}[ & \left.\operatorname{Tr}\left\{T^{n} \hat{U}_{z_{1}}^{\eta} \hat{U}_{z_{3}}^{\dagger \eta} T^{n} \hat{U}_{z_{3}}^{\eta} \hat{U}_{z_{2}}^{\dagger \eta}\right\}-N_{c} \operatorname{Tr}\left\{\hat{U}_{z_{1}}^{\eta} \hat{U}_{z_{2}}^{\dagger \eta}\right\}\right] \\
= & \frac{\alpha_{s}}{4 \pi^{2}} \int d^{2} z_{4}\left(\hat{U}_{z_{3}}^{\eta}\right)^{a a^{\prime}}\left[\frac { z _ { 1 2 } ^ { 2 } } { z _ { 1 4 } ^ { 2 } z _ { 2 4 } ^ { 2 } } \operatorname { T r } \left\{T^{a} T^{b} \hat{U}_{z_{1}}^{\eta} T^{a^{\prime}} T^{b^{\prime}} \hat{U}_{z_{2}}^{\dagger \eta}\right.\right. \\
& \left.+T^{b} T^{a} \hat{U}_{z_{1}}^{\eta} T^{b^{\prime}} T^{a^{\prime}} \hat{U}_{z_{2}}^{\dagger \eta}\right\}\left(2 \hat{U}_{z_{4}}^{\eta}-\hat{U}_{z_{1}}^{\eta}-\hat{U}_{z_{2}}^{\eta}\right)^{b b^{\prime}} \\
& -\frac{z_{13}^{2}}{z_{14}^{2} z_{34}^{2}} \operatorname{Tr}\left\{T^{a} T^{b} \hat{U}_{z_{1}}^{\eta}\left[T^{a^{\prime}}, T^{b^{\prime}}\right] \hat{U}_{z_{2}}^{\dagger \eta}\right. \\
& \left.+\left[T^{b}, T^{a}\right] \hat{U}_{z_{1}}^{\eta} T^{b^{\prime}} T^{a^{\prime}} \hat{U}_{z_{2}}^{\dagger \eta}\right\}\left(2 \hat{U}_{z_{4}}^{\eta}-\hat{U}_{z_{1}}^{\eta}-\hat{U}_{z_{3}}^{\eta}\right)^{b b^{\prime}} \\
& -\frac{z_{23}^{2}}{z_{24}^{2} z_{34}^{2}} \operatorname{Tr}\left\{\left[T^{a}, T^{b}\right] \hat{U}_{z_{1}}^{\eta} T^{a^{\prime}} T^{b^{\prime}} \hat{U}_{z_{2}}^{\dagger \eta}\right. \\
& \left.\left.+T^{b} T^{a} \hat{U}_{z_{1}}^{\eta}\left[T^{b^{\prime}}, T^{a^{\prime}}\right] \hat{U}_{z_{2}}^{\dagger \eta}\right\}\left(2 \hat{U}_{z_{4}}^{\eta}-\hat{U}_{z_{2}}^{\eta}-\hat{U}_{z_{3}}^{\eta}\right)^{b b^{\prime}}\right] \\
& -\frac{\alpha_{s} N_{c}}{\pi^{2}} \int d^{2} z_{4} \frac{z_{12}^{2}}{z_{14}^{2} z_{24}^{2}}\left(\operatorname{Tr}\left\{T^{a} \hat{U}_{z_{1}}^{\eta} \hat{U}_{z_{4}}^{\dagger \eta} T^{a} \hat{U}_{z_{4}}^{\eta} \hat{U}_{z_{2}}^{\dagger \eta}\right\}-N_{c} \operatorname{Tr}\left\{U_{z_{1}} U_{z_{2}}^{\dagger}\right\}\right) .
\end{aligned}
$$

\section{Appendix D. Color traces}

In this section we rewrite the adjoint traces in our evolution equation (6) in terms of the fundamental traces. The master formula for traces has the form (in this section we use the spacesaving notations $U_{i} \equiv \hat{U}_{z_{i}}$ )

$$
\begin{aligned}
\operatorname{Tr}\{[ & \left.\left.T^{a}, T^{b}\right] U_{1}^{\eta} T^{a^{\prime}} T^{b^{\prime}} U_{2}^{\dagger}+T^{b} T^{a} U_{z_{1}}^{\eta}\left[T^{b^{\prime}}, T^{a^{\prime}}\right] U_{z_{2}}^{\dagger}\right\} U_{3}^{a a^{\prime}} U_{4}^{b b^{\prime}} \\
= & \frac{1}{2}\left[-\operatorname{tr}\left\{U_{1} U_{2}^{\dagger}\right\} \operatorname{tr}\left\{U_{2} U_{4}^{\dagger}\right\} \operatorname{tr}\left\{U_{4} U_{3}^{\dagger}\right\} \operatorname{tr}\left\{U_{3} U_{1}^{\dagger}\right\}\right. \\
& -\operatorname{tr}\left\{U_{1}^{\dagger} U_{2}\right\} \operatorname{tr}\left\{U_{2}^{\dagger} U_{4}\right\} \operatorname{tr}\left\{U_{4}^{\dagger} U_{3}\right\} \operatorname{tr}\left\{U_{3}^{\dagger} U_{1}\right\}+\operatorname{tr}\left\{U_{1} U_{2}^{\dagger}\right\} \operatorname{tr}\left\{U_{2} U_{4}^{\dagger} U_{3} U_{1}^{\dagger} U_{4} U_{3}^{\dagger}\right\} \\
& +\operatorname{tr}\left\{U_{1} U_{3}^{\dagger}\right\} \operatorname{tr}\left\{U_{3} U_{2}^{\dagger} U_{4} U_{1}^{\dagger} U_{2} U_{4}^{\dagger}\right\}+\operatorname{tr}\left\{U_{3} U_{4}^{\dagger}\right\} \operatorname{tr}\left\{U_{4} U_{2}^{\dagger} U_{1} U_{3}^{\dagger} U_{2} U_{1}^{\dagger}\right\} \\
& +\operatorname{tr}\left\{U_{2} U_{4}^{\dagger}\right\} \operatorname{tr}\left\{U_{4} U_{1}^{\dagger} U_{3} U_{2}^{\dagger} U_{1} U_{3}^{\dagger}\right\}-\operatorname{tr}\left\{U_{1} U_{4}^{\dagger}\right\} \operatorname{tr}\left\{U_{4} U_{2}^{\dagger} U_{3} U_{1}^{\dagger} U_{2} U_{3}^{\dagger}\right\} \\
& -\operatorname{tr}\left\{U_{2} U_{3}^{\dagger}\right\} \operatorname{tr}\left\{U_{3} U_{1}^{\dagger} U_{4} U_{2}^{\dagger} U_{1} U_{4}^{\dagger}\right\}-\operatorname{tr}\left\{U_{1} U_{2}^{\dagger} U_{4} U_{3}^{\dagger}\right\} \operatorname{tr}\left\{U_{1}^{\dagger} U_{2} U_{4}^{\dagger} U_{3}\right\} \\
& +\operatorname{tr}\left\{U_{1}^{\dagger} U_{2}\right\} \operatorname{tr}\left\{U_{2}^{\dagger} U_{4} U_{3}^{\dagger} U_{1} U_{4}^{\dagger} U_{3}\right\}+\operatorname{tr}\left\{U_{1}^{\dagger} U_{3}\right\} \operatorname{tr}\left\{U_{3}^{\dagger} U_{2} U_{4}^{\dagger} U_{1} U_{2}^{\dagger} U_{4}\right\} \\
& +\operatorname{tr}\left\{U_{3}^{\dagger} U_{4}\right\} \operatorname{tr}\left\{U_{4}^{\dagger} U_{2} U_{1}^{\dagger} U_{3} U_{2}^{\dagger} U_{1}\right\}+\operatorname{tr}\left\{U_{2}^{\dagger} U_{4}\right\} \operatorname{tr}\left\{U_{4}^{\dagger} U_{1} U_{3}^{\dagger} U_{2} U_{1}^{\dagger} U_{3}\right\} \\
& -\operatorname{tr}\left\{U_{1}^{\dagger} U_{4}\right\} \operatorname{tr}\left\{U_{4}^{\dagger} U_{2} U_{3}^{\dagger} U_{1} U_{2}^{\dagger} U_{3}\right\}-\operatorname{tr}\left\{U_{2}^{\dagger} U_{3}\right\} \operatorname{tr}\left\{U_{3}^{\dagger} U_{1} U_{4}^{\dagger} U_{2} U_{1}^{\dagger} U_{4}\right\} \\
& \left.-\operatorname{tr}\left\{U_{1}^{\dagger} U_{2} U_{4}^{\dagger} U_{3}\right\} \operatorname{tr}\left\{U_{1} U_{2}^{\dagger} U_{4} U_{3}^{\dagger}\right\}\right],
\end{aligned}
$$


where $\operatorname{Tr}$ and $\operatorname{tr}$ stand for the trace in the adjoint and the fundamental representation, respectively. We will also need traces

$$
\begin{aligned}
\operatorname{Tr}\{ & \left.T^{a} U_{1} T^{b} U_{2}^{\dagger}\right\} U_{3}^{a b} \\
= & \operatorname{Tr}\left\{T^{a} U_{1} U_{3}^{\dagger} T^{b} U_{3} U_{2}^{\dagger}\right\} \\
= & \frac{1}{2} \operatorname{tr}\left[\left\{U_{1} U_{2}^{\dagger}\right\} \operatorname{tr}\left\{U_{2} U_{3}^{\dagger}\right\} \operatorname{tr}\left\{U_{3} U_{1}^{\dagger}\right\}+\operatorname{tr}\left\{U_{1} U_{3}^{\dagger}\right\} \operatorname{tr}\left\{U_{3} U_{2}^{\dagger}\right\} \operatorname{tr}\left\{U_{2} U_{1}^{\dagger}\right\}\right. \\
& \left.\quad-\operatorname{tr}\left\{U_{1} U_{2}^{\dagger} U_{3} U_{1}^{\dagger} U_{2} U_{3}^{\dagger}\right\}-\operatorname{tr}\left\{U_{1} U_{3}^{\dagger} U_{2} U_{1}^{\dagger} U_{3} U_{2}^{\dagger}\right\}\right], \\
\operatorname{Tr}\{ & {\left.\left[T^{a}, T^{b}\right] U_{1} T^{a^{\prime}} T^{b^{\prime}} U_{2}^{\dagger}+T^{b} T^{a} U_{1}\left[T^{b^{\prime}}, T^{a^{\prime}}\right] U_{2}^{\dagger}\right\} U_{3}^{a a^{\prime}} U_{3}^{b b^{\prime}} } \\
= & \operatorname{Tr}\left\{\left[T^{a}, T^{b}\right] U_{1}^{\eta} T^{a^{\prime}} T^{b^{\prime}} U_{2}^{\dagger}+T^{b} T^{a} U_{2}^{\eta}\left[T^{b^{\prime}}, T^{a^{\prime}}\right] U_{2}^{\dagger}\right\} U_{1}^{a a^{\prime}} U_{3}^{b b^{\prime}} \\
= & \operatorname{Tr}\left\{\left[T^{a}, T^{b}\right] U_{1}^{\eta} T^{a^{\prime}} T^{b^{\prime}} U_{2}^{\dagger}+T^{b} T^{a} U_{1}^{\eta}\left[T^{b^{\prime}}, T^{a^{\prime}}\right] U_{2}^{\dagger}\right\} U_{3}^{a a^{\prime}} U_{2}^{b b^{\prime}} \\
= & -N_{c} \operatorname{Tr}\left\{T^{a} U_{1} U_{3}^{\dagger} T^{a} U_{3} U_{2}^{\dagger}\right\}, \\
\operatorname{Tr}\{ & \left.T^{a} T^{b} U_{1} T^{a^{\prime}} T^{b^{\prime}} U_{2}^{\dagger}+T^{b} T^{a} U_{1} T^{b^{\prime}} T^{a^{\prime}} U_{2}^{\dagger}\right\} U_{1}^{a a^{\prime}} U_{3}^{b b^{\prime}} \\
= & \operatorname{Tr}\left\{T^{a} T^{b} U_{1} T^{a^{\prime}} T^{b^{\prime}} U_{2}^{\dagger}+T^{b} T^{a} U_{1} T^{b^{\prime}} T^{a^{\prime}} U_{2}^{\dagger}\right\} U_{2}^{a a^{\prime}} U_{3}^{b b^{\prime}} \\
= & N_{c} \operatorname{Tr}\left\{T^{a} U_{1} U_{3}^{\dagger} T^{a} U_{3} U_{2}^{\dagger}\right\},
\end{aligned}
$$

which easily follow from Eq. (106).

Let us now find the master trace (106) in the two-gluon (BFKL) approximation. First, note that in this approximation the trace of six Wilson lines reduces to one dipole. Indeed,

$$
\begin{aligned}
\operatorname{tr} & \left\{U_{1} U_{3}^{\dagger} U_{4} U_{2}^{\dagger} U_{3} U_{4}^{\dagger}\right\} \\
& =\operatorname{tr}\left\{\left(U_{1} U_{3}^{\dagger}-1\right) U_{4}\left(U_{2}^{\dagger} U_{3}-1\right) U_{4}^{\dagger}\right\}+\operatorname{tr}\left\{U_{1} U_{3}^{\dagger}-1\right\}+\operatorname{tr}\left\{U_{2}^{\dagger} U_{3}-1\right\}+N_{c}^{2} \\
& =\operatorname{tr}\left\{\left(U_{1} U_{3}^{\dagger}-1\right) U_{3}\left(U_{2}^{\dagger} U_{3}-1\right) U_{3}^{\dagger}\right\}+\operatorname{tr}\left\{U_{1} U_{3}^{\dagger}-1\right\}+\operatorname{tr}\left\{U_{2}^{\dagger} U_{3}-1\right\}+N_{c}^{2} \\
& =\operatorname{tr}\left\{U_{1} U_{2}^{\dagger}\right\},
\end{aligned}
$$

where we replaced by $U_{4}$ by $U_{3}$ in the first term in the second line since it does not matter in the two-gluon approximation (both of them should be replaced by 1). All other six-Wilson-line terms in the r.h.s. of Eq. (106) can be similarly reduced to single-dipole terms.

Using the same trick we can reduce the trace of four Wilson lines to the sum of dipoles:

$$
\begin{aligned}
\operatorname{tr}\{ & \left.U_{1} U_{2}^{\dagger} U_{4} U_{3}^{\dagger}\right\} \\
= & \operatorname{tr}\left\{\left(U_{1} U_{2}^{\dagger}-1\right)\left(U_{4} U_{3}^{\dagger}-1\right)\right\}+\operatorname{tr}\left\{U_{1} U_{2}^{\dagger}-1\right\}+\operatorname{tr}\left\{U_{4} U_{3}^{\dagger}-1\right\}+N_{c}^{2} \\
= & \operatorname{tr}\left\{\left(U_{1} U_{2}^{\dagger}-1\right) U_{2} U_{4}^{\dagger}\left(U_{4} U_{3}^{\dagger}-1\right)\right\}+\operatorname{tr}\left\{U_{1} U_{2}^{\dagger}-1\right\}+\operatorname{tr}\left\{U_{4} U_{3}^{\dagger}-1\right\}+N_{c}^{2} \\
= & \operatorname{tr}\left\{\left(U_{1} U_{2}^{\dagger}-1\right)\right\}+\operatorname{tr}\left\{U_{2} U_{4}^{\dagger}-1\right\}+\operatorname{tr}\left\{U_{4} U_{3}^{\dagger}-1\right\}+\operatorname{tr}\left\{U_{1} U_{3}^{\dagger}-1\right\} \\
& -\operatorname{tr}\left\{U_{1} U_{4}^{\dagger}-1\right\}-\operatorname{tr}\left\{U_{2} U_{3}^{\dagger}-1\right\}+N_{c}^{2},
\end{aligned}
$$

where in the first term in the second line we have inserted $U_{2} U_{4}^{\dagger}$ since it does not matter in the two-gluon approximation.

Using formulas (108) and (109) it is easy to demonstrate that all the terms containing traces of four and six Wilson lines in the r.h.s. of Eq. (106) sum to $2 N_{c}^{2}$ in the two-gluon approximation 
so one gets

$$
\begin{aligned}
& \operatorname{Tr}\{[\left.\left.T^{a}, T^{b}\right] U_{1}^{\eta} T^{a^{\prime}} T^{b^{\prime}} U_{2}^{\dagger}+T^{b} T^{a} U_{z_{1}}^{\eta}\left[T^{b^{\prime}}, T^{a^{\prime}}\right] U_{z_{2}}^{\dagger}\right\} U_{3}^{a a^{\prime}} U_{4}^{b b^{\prime}} \\
& \stackrel{\text { 2-gluon }}{=}-N_{c}^{2}\left(N_{c}^{2}-1\right)-\frac{1}{2} N_{c}^{3}\left[\operatorname{tr}\left\{U_{1} U_{2}^{\dagger}-1\right\}+\operatorname{tr}\left\{U_{1}^{\dagger} U_{2}-1\right\}+\operatorname{tr}\left\{U_{2} U_{4}^{\dagger}-1\right\}\right. \\
&\left.\quad+\operatorname{tr}\left\{U_{2}^{\dagger} U_{4}-1\right\}+\operatorname{tr}\left\{U_{4} U_{3}^{\dagger}-1\right\}+\operatorname{tr}\left\{U_{4}^{\dagger} U_{3}-1\right\}+\operatorname{tr}\left\{U_{3} U_{1}^{\dagger}-1\right\}+\operatorname{tr}\left\{U_{3}^{\dagger} U_{1}\right\}\right] \\
&=-N_{c}^{2}\left(N_{c}^{2}-1\right)+\frac{1}{2} N_{c}^{2}\left(N_{c}^{2}-1\right)\left[\hat{\mathcal{V}}\left(z_{1}, z_{2}\right)+\hat{\mathcal{V}}\left(z_{1}, z_{3}\right)+\hat{\mathcal{V}}\left(z_{2}, z_{4}\right)+\hat{\mathcal{V}}\left(z_{3}, z_{4}\right)\right],
\end{aligned}
$$

where we use the definition (54). For completeness, let us present also $\operatorname{Tr}\left\{T^{a} U_{1} U_{3}^{\dagger} T^{b} U_{3} U_{2}^{\dagger}\right\}$ in the two-gluon approximation. From the first line in Eq. (107) one easily obtains

$$
\begin{aligned}
& \operatorname{Tr}\left\{T^{a} U_{1} U_{3}^{\dagger} T^{b} U_{3} U_{2}^{\dagger}\right\} \\
& \stackrel{2 \text {-gluon }}{=} N_{c}\left(N_{c}^{2}-1\right)-\frac{1}{2} N_{c}\left(N_{c}^{2}-1\right)\left[\hat{\mathcal{V}}\left(z_{1}, z_{2}\right)+\hat{\mathcal{V}}\left(z_{1}, z_{3}\right)+\hat{\mathcal{V}}\left(z_{2}, z_{3}\right)\right] .
\end{aligned}
$$

To describe the conformal operator (48) we need one more trace (which eventually drops out after the integration of the operator (48) over $z_{3}$ with the weight $\frac{z_{12}^{2}}{z_{13}^{2} z_{23}^{2}}$ )

$$
\begin{aligned}
\operatorname{Tr}\{ & \left.T^{a} T^{b} U_{1} T^{a^{\prime}} T^{b^{\prime}} U_{2}^{\dagger}\right\} U_{3}^{a a^{\prime}} U_{4}^{b b^{\prime}} \\
= & \frac{1}{4}\left[\operatorname{tr}\left\{U_{1} U_{3}^{\dagger}\right\} \operatorname{tr}\left\{U_{3} U_{2}^{\dagger}\right\} \operatorname{tr}\left\{U_{2} U_{4}^{\dagger}\right\} \operatorname{tr}\left\{U_{4} U_{1}^{\dagger}\right\}\right. \\
& +\operatorname{tr}\left\{U_{3} U_{1}^{\dagger}\right\} \operatorname{tr}\left\{U_{2} U_{3}^{\dagger}\right\} \operatorname{tr}\left\{U_{4} U_{2}^{\dagger}\right\} \operatorname{tr}\left\{U_{4}^{\dagger} U_{1}\right\} \\
& +\operatorname{tr}\left\{U_{3} U_{1}^{\dagger} U_{4} U_{2}^{\dagger}\right\} \operatorname{tr}\left\{U_{1} U_{4}^{\dagger} U_{2} U_{3}^{\dagger}\right\}+\operatorname{tr}\left\{U_{2} U_{4}^{\dagger} U_{1} U_{3}^{\dagger}\right\} \operatorname{tr}\left\{U_{1}^{\dagger} U_{3} U_{2}^{\dagger} U_{4}\right\} \\
& +\operatorname{tr}\left\{U_{4} U_{3}^{\dagger}\right\} \operatorname{tr}\left\{U_{3} U_{1}^{\dagger} U_{2} U_{4}^{\dagger} U_{1} U_{2}^{\dagger}\right\}+\operatorname{tr}\left\{U_{1} U_{2}^{\dagger}\right\} \operatorname{tr}\left\{U_{4}^{\dagger} U_{3} U_{1}^{\dagger} U_{4} U_{3}^{\dagger} U_{2}\right\} \\
& +\operatorname{tr}\left\{U_{4}^{\dagger} U_{3}\right\} \operatorname{tr}\left\{U_{2} U_{1}^{\dagger} U_{4} U_{2}^{\dagger} U_{1} U_{3}^{\dagger}\right\}+\operatorname{tr}\left\{U_{1}^{\dagger} U_{2}\right\} \operatorname{tr}\left\{U_{3} U_{4}^{\dagger} U_{1} U_{3}^{\dagger} U_{4} U_{2}^{\dagger}\right\} \\
& -\operatorname{tr}\left\{U_{1} U_{3}^{\dagger}\right\} \operatorname{tr}\left\{U_{2} U_{1}^{\dagger} U_{4} U_{2}^{\dagger} U_{3} U_{4}^{\dagger}\right\}-\operatorname{tr}\left\{U_{1}^{\dagger} U_{4}\right\} \operatorname{tr}\left\{U_{1} U_{3}^{\dagger} U_{2} U_{4}^{\dagger} U_{3} U_{2}^{\dagger}\right\} \\
& -\operatorname{tr}\left\{U_{3} U_{2}^{\dagger}\right\} \operatorname{tr}\left\{U_{1}^{\dagger} U_{2} U_{4}^{\dagger} U_{1} U_{3}^{\dagger} U_{4}\right\}-\operatorname{tr}\left\{U_{2} U_{4}^{\dagger}\right\} \operatorname{tr}\left\{U_{1} U_{2}^{\dagger} U_{3} U_{1}^{\dagger} U_{4} U_{3}^{\dagger}\right\} \\
& -\operatorname{tr}\left\{U_{3} U_{1}^{\dagger}\right\} \operatorname{tr}\left\{U_{4} U_{3}^{\dagger} U_{2} U_{4}^{\dagger} U_{1} U_{2}^{\dagger}\right\}-\operatorname{tr}\left\{U_{4} U_{2}^{\dagger}\right\} \operatorname{tr}\left\{U_{1}^{\dagger} U_{3} U_{4}^{\dagger} U_{1} U_{3}^{\dagger} U_{2}\right\} \\
& \left.-\operatorname{tr}\left\{U_{2} U_{3}^{\dagger}\right\} \operatorname{tr}\left\{U_{1} U_{4}^{\dagger} U_{3} U_{1}^{\dagger} U_{4} U_{2}^{\dagger}\right\}-\operatorname{tr}\left\{U_{4}^{\dagger} U_{1}\right\} \operatorname{tr}\left\{U_{2} U_{3}^{\dagger} U_{4} U_{2}^{\dagger} U_{3} U_{1}^{\dagger}\right\}\right] .
\end{aligned}
$$

In the two-gluon approximation this yields

$$
\begin{aligned}
\operatorname{Tr} & \left\{T^{a} T^{b} U_{1} T^{a^{\prime}} T^{b^{\prime}} U_{2}^{\dagger}\right\} U_{3}^{a a^{\prime}} U_{4}^{b b^{\prime}} \\
\quad= & \frac{1}{2} N_{c}^{2}\left(N_{c}^{2}-1\right)-\frac{1}{4} N_{c}^{2}\left(N_{c}^{2}-1\right)\left[\hat{\mathcal{V}}\left(z_{1}, z_{3}\right)+\hat{\mathcal{V}}\left(z_{2}, z_{3}\right)+\hat{\mathcal{V}}\left(z_{1}, z_{4}\right)+\hat{\mathcal{V}}\left(z_{2}, z_{4}\right)\right] .
\end{aligned}
$$

\section{Appendix E. Integrals}

In this section we calculate two basic integrals which we use in this paper. The first one is

$$
\frac{z_{12}^{2}}{z_{13}^{2} z_{23}^{2}} \int \frac{d^{2} z_{4}}{\pi}\left[\frac{z_{13}^{2}}{z_{14}^{2} z_{34}^{2}}+\frac{z_{23}^{2}}{z_{24}^{2} z_{34}^{2}}-\frac{z_{12}^{2}}{z_{14}^{2} z_{24}^{2}}\right] \ln \frac{z_{13}^{2} z_{24}^{2}}{z_{14}^{2} z_{23}^{2}}=4 \zeta(3)\left[\delta\left(z_{23}\right)-\delta\left(z_{13}\right)\right] .
$$


The easiest way to prove this at $z_{3} \neq z_{1}, z_{2}$ is to set $z_{2}=0$ and make an inversion $x \rightarrow 1 / \tilde{x}$ so the integral (114) reduces to

$$
2\left(\tilde{z}_{1}-\tilde{z}_{2}\right)^{2} \int d^{2} \tilde{z}_{4} \frac{\left(\tilde{z}_{1}-\tilde{z}_{3}, \tilde{z}_{1}-\tilde{z}_{4}\right)}{\left(\tilde{z}_{1}-\tilde{z}_{4}\right)^{2}\left(\tilde{z}_{3}-\tilde{z}_{4}\right)^{2}} \ln \frac{\left(\tilde{z}_{1}-\tilde{z}_{3}\right)^{2}}{\left(\tilde{z}_{1}-\tilde{z}_{4}\right)^{2}}=0 .
$$

The $\delta$-function terms in the r.h.s. of Eq. (114) can be restored from the formula

$$
\int \frac{d^{2} z_{3} d^{2} z_{4}}{\pi^{2}}\left[\frac{\left(z_{12}, z_{23}\right)}{z_{34}^{2}}\left(\frac{1}{z_{13}^{2} z_{24}^{2}}+\frac{1}{z_{23}^{2} z_{14}^{2}}\right)-\frac{z_{12}^{2}\left(z_{12}, z_{23}\right)}{z_{13}^{2} z_{24}^{2} z_{14}^{2} z_{23}^{2}}\right] \ln \frac{z_{13}^{2} z_{24}^{2}}{z_{23}^{2} z_{14}^{2}}=4 \zeta(3),
$$

which follows from the integral

$$
\begin{aligned}
\frac{1}{\pi} \int & d^{d} z d^{d} z^{\prime}\left[\frac{(x, z)}{(x-z)^{2}\left(z-z^{\prime}\right)^{2} z^{\prime 2}}+\frac{(x, z)}{\left(x-z^{\prime}\right)^{2}\left(z-z^{\prime}\right)^{2} z^{2}}\right. \\
& \left.-\frac{x^{2}(x, z)}{(x-z)^{2} z^{2}\left(x-z^{\prime}\right)^{2} z^{\prime 2}}\right] \ln \frac{(x-z)^{2} z^{\prime 2}}{\left(x-z^{\prime}\right)^{2} z^{2}} \\
= & B\left(\frac{d}{2}, \frac{d}{2}-1\right) B\left(d-1, \frac{d}{2}-1\right) \frac{\Gamma(3-d)}{\left(x^{2}\right)^{2-d}}\left\{3 \psi\left(\frac{d}{2}-1\right)-2 \psi(d-2)+2 \psi(1)\right. \\
& \left.-2 \psi\left(2-\frac{d}{2}\right)-\psi\left(\frac{d}{2}\right)+\frac{4}{d-2} \frac{\Gamma\left(\frac{d}{2}\right) \Gamma\left(3 \frac{d}{2}-2\right) \Gamma^{2}\left(2-\frac{d}{2}\right)}{\Gamma^{2}(d-1) \Gamma(3-d)}\right\} \stackrel{d \rightarrow 2}{\rightarrow}-4 \zeta(3) .
\end{aligned}
$$

The second integral is somewhat more tedious

$$
\begin{aligned}
& \frac{z_{12}^{2}}{z_{13}^{2} z_{23}^{2}} \int \frac{d^{2} z_{4}}{\pi}\left[\frac{z_{23}^{2}}{z_{34}^{2} z_{24}^{2}} \ln \frac{z_{12}^{2} z_{13}^{2} z_{34}^{2}}{z_{14}^{4} z_{24}^{2}}+\frac{z_{13}^{2}}{z_{14}^{2} z_{34}^{2}} \ln \frac{z_{13}^{4} z_{23}^{2}}{z_{12}^{2} z_{14}^{2} z_{34}^{2}}+\frac{z_{12}^{2}}{z_{14}^{2} z_{24}^{2}} \ln \frac{z_{14}^{2} z_{24}^{2}}{z_{13}^{2} z_{23}^{2}}\right] \\
& \quad=2 \frac{z_{12}^{2}}{z_{13}^{2} z_{23}^{2}} \ln \frac{z_{12}^{2}}{z_{13}^{2}} \ln \frac{z_{12}^{2}}{z_{23}^{2}}-8 \zeta(3) \delta\left(z_{13}\right) .
\end{aligned}
$$

First we prove this equation at $z_{3} \neq z_{1}, z_{2}$. To simplify the notations, we take $z_{2}=0$ an denote $z_{1} \equiv x, z_{3} \equiv z$ and $z_{4} \equiv z^{\prime}$. Let us start from the first term in the square brackets in the 1.h.s. of Eq. (116)

$$
\begin{aligned}
\int d^{2} z^{\prime} & \frac{z^{2}}{\left(z-z^{\prime}\right)^{2} z^{\prime 2}} \ln \frac{x^{2}(x-z)^{2}\left(z-z^{\prime}\right)^{2}}{\left(x-z^{\prime}\right)^{4} z^{\prime 2}} \\
= & -2 \int d^{2} z^{\prime}\left[\frac{1}{\left(z-z^{\prime}\right)^{2}}-\frac{1}{z^{\prime 2}}\right] \ln \frac{\left(x-z^{\prime}\right)^{2}}{x^{2}} \\
& -\ln \frac{x^{2}}{(x-z)^{2}} \int d^{2} z^{\prime} \frac{z^{2}}{\left(z-z^{\prime}\right)^{2} z^{\prime 2}}-4 \int d^{2} z^{\prime} \frac{\left(z, z-z^{\prime}\right)}{\left(z-z^{\prime}\right)^{2} z^{\prime 2}} \ln \frac{\left(x-z^{\prime}\right)^{2}}{x^{2}} .
\end{aligned}
$$

We get

$$
\begin{aligned}
& -2 \int d^{2} z^{\prime}\left[\frac{1}{\left(z-z^{\prime}\right)^{2}}-\frac{1}{z^{\prime 2}}\right] \ln \frac{\left(x-z^{\prime}\right)^{2}}{x^{2}}-\ln \frac{x^{2}}{(x-z)^{2}} \int d^{2} z^{\prime} \frac{z^{2}}{\left(z-z^{\prime}\right)^{2} z^{\prime 2}} \\
& =\lim _{d \rightarrow 2}\left(-2 \int d^{d} z^{\prime}\left[\frac{1}{\left(z-z^{\prime}\right)^{2}}-\frac{1}{z^{\prime 2}}\right] \ln \frac{\left(x-z^{\prime}\right)^{2}}{x^{2}}-\ln \frac{x^{2}}{(x-z)^{2}} \int d^{d} z^{\prime} \frac{z^{2}}{\left(z-z^{\prime}\right)^{2} z^{\prime 2}}\right)
\end{aligned}
$$




$$
\begin{aligned}
& =\lim _{d \rightarrow 2} 2 \pi\left[\frac{\Gamma\left(1-\frac{d}{2}\right)}{\left((x-z)^{2}\right)^{1-\frac{d}{2}}}-\frac{\Gamma\left(1-\frac{d}{2}\right)}{\left(x^{2}\right)^{1-\frac{d}{2}}}-\frac{\Gamma\left(2-\frac{d}{2}\right)}{\left(z^{2}\right)^{1-\frac{d}{2}}} \ln \frac{x^{2}}{(x-z)^{2}}\right] B\left(\frac{d}{2}, \frac{d}{2}-1\right) \\
& =-\pi \ln \frac{(x-z)^{2} x^{2}}{z^{4}} \ln \frac{(x-z)^{2}}{x^{2}}
\end{aligned}
$$

and

$$
\begin{aligned}
-4 & \int d^{d} z^{\prime} \frac{\left(z, z-z^{\prime}\right)}{\left(z-z^{\prime}\right)^{2} z^{\prime 2}} \ln \frac{\left(x-z^{\prime}\right)^{2}}{x^{2}} \\
= & \lim _{d \rightarrow 2} 4 \frac{\pi^{d / 2}}{d-2} \frac{\Gamma(d / 2)}{\Gamma(d-1)} \Gamma\left(\frac{d}{2}-1\right) \Gamma\left(2-\frac{d}{2}\right)\left[1+\left(\frac{d}{2}-1\right) \ln z^{2}\right. \\
& +\left(\frac{d}{2}-1\right) \ln z^{2}+\frac{1}{2}\left(\frac{d}{2}-1\right)^{2}\left(\ln ^{2} \frac{z^{2} x^{2}}{(x-z)^{2}}-\ln ^{2} \frac{x^{4}}{(x-z)^{2}}+\ln ^{2} x^{2}\right) \\
& \left.-1-\left(\frac{d}{2}-1\right) \ln z^{2}-\frac{1}{2}\left(\frac{d}{2}-1\right)^{2} \ln ^{2} z^{2}\right] \\
= & 2 \pi \ln \frac{x^{2}}{z^{2}} \ln \frac{(x-z)^{2}}{x^{2}},
\end{aligned}
$$

so

$$
\int d^{2} z^{\prime} \frac{z^{2}}{\left(z-z^{\prime}\right)^{2} z^{\prime 2}} \ln \frac{x^{2}(x-z)^{2}}{\left(x-z^{\prime}\right)^{4}}=-\pi \ln ^{2} \frac{(x-z)^{2}}{x^{2}} .
$$

Similarly

$$
\begin{aligned}
\int d^{2} z^{\prime} & {\left[-\frac{(x-z)^{2}}{\left(x-z^{\prime}\right)^{2}\left(z-z^{\prime}\right)^{2}} \ln \frac{x^{2}\left(x-z^{\prime}\right)^{2}\left(z-z^{\prime}\right)^{2}}{(x-z)^{4} z^{2}}+\frac{x^{2}}{\left(x-z^{\prime}\right)^{2} z^{\prime 2}} \ln \frac{\left(x-z^{\prime}\right)^{2} z^{\prime 2}}{(x-z)^{2} z^{2}}\right] } \\
= & \lim _{d \rightarrow 2} 2 \pi \frac{\Gamma\left(2-\frac{d}{2}\right) \Gamma^{2}\left(\frac{d}{2}\right)}{\left(\frac{d}{2}-1\right) \Gamma(d-1)}\left\{|x-z|^{d-2} \ln \frac{(x-z)^{4} z^{2}}{x^{2}}\right. \\
& -2|x-z|^{d-2}\left[-\frac{1}{d-2}+\ln (x-z)^{2}-\psi\left(2-\frac{d}{2}\right)+\psi\left(\frac{d}{2}\right)+\psi(1)-\psi(d-1)\right] \\
& +2|x|^{d-2}\left[-\frac{1}{d-2}+\ln x^{2}-\psi\left(2-\frac{d}{2}\right)+\psi\left(\frac{d}{2}\right)+\psi(1)-\psi(d-1)\right] \\
& \left.-|x|^{d-2} \ln (x-z)^{2} z^{2}\right\} \\
= & \pi \ln ^{2} \frac{x^{2}}{(x-z)^{2}}+2 \pi \ln \frac{x^{2}}{z^{2}} \ln \frac{x^{2}}{(x-z)^{2}}
\end{aligned}
$$

and therefore

$$
\begin{aligned}
\int d^{2} z^{\prime} & {\left[-\frac{z^{2}}{\left(z-z^{\prime}\right)^{2} z^{\prime 2}} \ln \frac{\left(x-z^{\prime}\right)^{4}}{x^{2}(x-z)^{2}}+\frac{(x-z)^{2}}{\left(x-z^{\prime}\right)^{2}\left(z-z^{\prime}\right)^{2}} \ln \frac{(x-z)^{4} z^{2}}{x^{2}\left(x-z^{\prime}\right)^{2}\left(z-z^{\prime}\right)^{2}}\right.} \\
+ & \left.\frac{x^{2}}{\left(x-z^{\prime}\right)^{2} z^{\prime 2}} \ln \frac{\left(x-z^{\prime}\right)^{2} z^{\prime 2}}{(x-z)^{2} z^{2}}\right] \\
= & 2 \pi \ln \frac{x^{2}}{z^{2}} \ln \frac{x^{2}}{(x-z)^{2}} .
\end{aligned}
$$


However, it is easy to see that some sort of $\delta$-function contribution to the r.h.s. is necessary. If we integrate the 1.h.s. over $z$ with the weight $\frac{x^{2}}{(x-z)^{2} z^{2}}$ we get zero because of the antisymmetry of the integrand with respect to $z \leftrightarrow z^{\prime}$. On the other hand, the integral of the r.h.s. does not vanish because

$$
\int d^{2} z \frac{x^{2}}{(x-z)^{2} z^{2}} \ln \frac{x^{2}}{z^{2}} \ln \frac{x^{2}}{(x-z)^{2}}=4 \pi \zeta(3) .
$$

To fix the coefficients in front of possible $\delta$-function contributions $\sim \delta(z)$ and/or $\delta(x-z)$ we calculate the integral of the 1.h.s. of Eq. (122) with the trial function $\frac{(x, x-z)}{z^{2}(x-z)^{2}}$. We get

$$
\begin{aligned}
\frac{1}{\pi^{2}} \int & d^{2} z d^{2} z^{\prime} \frac{(x, x-z)}{z^{2}(x-z)^{2}}\left[\frac{(x-z)^{2} \ln \frac{x^{2} z^{2}}{z^{\prime 4}}}{\left(x-z^{\prime}\right)^{2}\left(z-z^{\prime}\right)^{2}}-\frac{x^{2}}{\left(x-z^{\prime}\right)^{2} z^{\prime 2}} \ln \frac{z^{2}(x-z)^{2}}{z^{\prime 2}\left(x-z^{\prime}\right)^{2}}\right. \\
& \left.+\frac{z^{2}}{\left(z-z^{\prime}\right)^{2} z^{\prime 2}} \ln \frac{z^{4}(x-z)^{2}}{x^{2} z^{\prime 2}\left(z-z^{\prime}\right)^{2}}\right] \\
= & \lim _{d \rightarrow 2} B\left(\frac{d}{2}, \frac{d}{2}-1\right) B\left(d-1, \frac{d}{2}-1\right) \frac{\Gamma(3-d)}{\left(x^{2}\right)^{2-d}} \\
& \times\left\{\psi(1)+\psi\left(\frac{d}{2}\right)+\psi(3-d)+\psi\left(3 \frac{d}{2}-2\right)-2 \psi(d-1)-2 \psi\left(2-\frac{d}{2}\right)\right\} \\
= & -4 \zeta(3),
\end{aligned}
$$

where $B(a, b)=\Gamma(a) \Gamma(b) / \Gamma(a+b)$. It is clear now that the result for the integral in the 1.h.s. of the formula (116) should be as cited in the r.h.s. of Eq. (116) - it satisfies both Eq. (124) and the requirement that the integral of the 1.h.s. with the trial function $\frac{x^{2}}{z^{2}(x-z)^{2}}$ vanishes.

We will need one more integral which is obtained by antisymmetrization of Eq. (116) with respect to $z_{1} \leftrightarrow z_{2}$

$$
\frac{z_{12}^{2}}{z_{13}^{2} z_{23}^{2}} \int \frac{d^{2} z_{4}}{\pi}\left[\frac{z_{13}^{2}}{z_{14}^{2} z_{34}^{2}} \ln \frac{z_{12}^{2} z_{34}^{2}}{z_{13}^{2} z_{24}^{2}}-\frac{z_{23}^{2}}{z_{34}^{2} z_{24}^{2}} \ln \frac{z_{12}^{2} z_{34}^{2}}{z_{14}^{2} z_{23}^{2}}\right]=4 \zeta(3)\left[\delta\left(z_{13}\right)-\delta\left(z_{23}\right)\right] .
$$

\section{References}

[1] A.V. Kotikov, L.N. Lipatov, Nucl. Phys. B 582 (2000) 19; A.V. Kotikov, L.N. Lipatov, Nucl. Phys. B 661 (2003) 19; A.V. Kotikov, L.N. Lipatov, Nucl. Phys. B 685 (2004) 405, Erratum.

[2] I. Balitsky, High-energy QCD and Wilson Lines, in: M. Shifman (Ed.), At the Frontier of Particle Physics, vol. 2, World Scientific, Singapore, 2001, pp. 1237-1342, hep-ph/0101042.

[3] A.H. Mueller, Nucl. Phys. B 415 (1994) 373; A.H. Mueller, B. Patel, Nucl. Phys. B 425 (1994) 471.

[4] N.N. Nikolaev, B.G. Zakharov, Phys. Lett. B 332 (1994) 184;

N.N. Nikolaev, B.G. Zakharov, Z. Phys. C 64 (1994) 631;

N.N. Nikolaev, B.G. Zakharov, V.R. Zoller, JETP Lett. 59 (1994) 6.

[5] I. Balitsky, Nucl. Phys. B 463 (1996) 99;

I. Balitsky, Operator expansion for diffractive high-energy scattering, hep-ph/9706411.

[6] Yu.V. Kovchegov, Phys. Rev. D 60 (1999) 034008; Yu.V. Kovchegov, Phys. Rev. D 61 (2000) 074018.

[7] V.S. Fadin, E.A. Kuraev, L.N. Lipatov, Phys. Lett. B 60 (1975) 50;

I. Balitsky, L.N. Lipatov, Sov. J. Nucl. Phys. 28 (1978) 822. 
[8] L.V. Gribov, E.M. Levin, M.G. Ryskin, Phys. Rep. 100 (1983) 1; A.H. Mueller, J.W. Qiu, Nucl. Phys. B 268 (1986) 427; A.H. Mueller, Nucl. Phys. B 335 (1990) 115.

[9] E. Iancu, R. Venugopalan, in: R.C. Hwa, X.N. Wang (Eds.), Quark Gluon Plasma 3, World Scientific, Singapore, 2003, pp. 249-363, hep-ph/0303204;

H. Weigert, Prog. Part. Nucl. Phys. 55 (2005) 461;

J. Jalilian-Marian, Yu.V. Kovchegov, Prog. Part. Nucl. Phys. 56 (2006) 104.

[10] L.N. Lipatov, Sov. Phys. JETP 63 (1986) 904.

[11] I. Balitsky, G.A. Chirilli, Phys. Rev. D 77 (2008) 014019.

[12] J.M. Maldacena, Phys. Rev. Lett. 80 (1998) 4859.

[13] I. Balitsky, A.V. Belitsky, Nucl. Phys. B 629 (2002) 290.

[14] I. Balitsky, Phys. Rev. D 60 (1999) 014020.

[15] A.V. Belitsky, S.E. Derkachov, G.P. Korchemsky, A.N. Manashov, Phys. Rev. D 70 (2004) 045021.

[16] G. Altarelli, G. Curci, G. Martinelli, S. Petrarka, Nucl. Phys. B 187 (1981) 461.

[17] I. Balitsky, Phys. Rev. D 75 (2007) 014001.

[18] Yu.V. Kovchegov, H. Weigert, Nucl. Phys. A 784 (2007) 188.

[19] I. Balitsky, G.A. Chirilli, Phys. Rev. D 79 (2009) 031502.

[20] V.S. Fadin, L.N. Lipatov, Phys. Lett. B 429 (1998) 127; G. Camici, M. Ciafaloni, Phys. Lett. B 430 (1998) 349.

[21] V.S. Fadin, R. Fiore, Phys. Lett. B 661 (2008) 139.

[22] T. Altinoluk, A. Kovner, M. Lublinsky, Inclusive gluon production in the QCD reggeon field theory: Pomeron loops included, arXiv:0901.2560 [hep-ph];

A. Kovner, M. Lublinsky, Phys. Rev. D 71 (2005) 085004;

A. Kovner, M. Lublinsky, Phys. Rev. Lett. 94 (2005) 181603;

A. Kovner, M. Lublinsky, JHEP 0503 (2005) 001;

Y. Hatta, E. Iancu, L. McLerran, A. Stasto, D.N. Triantafyllopoulos, Nucl. Phys. A 764 (2006) 423;

I. Balitsky, Phys. Lett. B 518 (2001) 235;

I. Balitsky, Phys. Rev. D 72 (2005) 074027;

A.H. Mueller, A.I. Shoshi, S.M.H. Wong, Nucl. Phys. B 715 (2005) 440.

[23] L. Cornalba, M.S. Costa, J. Penedones, JHEP 0806 (2008) 48.

[24] E. D’Hoker, D.Z. Freedman, W. Skiba, Phys. Rev. D 59 (1999) 045008. 